TRANSACTIONS OF THE

AMERICAN MATHEMATICAL SOCIETY

Volume 356, Number 9, Pages 3621-3668

S 0002-9947(03)03373-7

Article electronically published on December 12, 2003

\title{
REALIZABILITY OF MODULES OVER TATE COHOMOLOGY
}

\author{
DAVID BENSON, HENNING KRAUSE, AND STEFAN SCHWEDE
}

\begin{abstract}
Let $k$ be a field and let $G$ be a finite group. There is a canonical element in the Hochschild cohomology of the Tate cohomology $\gamma_{G} \in$ $H H^{3,-1} \hat{H}^{*}(G, k)$ with the following property. Given a graded $\hat{H}^{*}(G, k)$-module $X$, the image of $\gamma_{G}$ in $\operatorname{Ext}_{\hat{H}^{*}(G, k)}^{3,-1}(X, X)$ vanishes if and only if $X$ is isomorphic to a direct summand of $\hat{H}^{*}(G, M)$ for some $k G$-module $M$.

The description of the realizability obstruction works in any triangulated category with direct sums. We show that in the case of the derived category of a differential graded algebra $A$, there is also a canonical element of Hochschild cohomology $H H^{3,-1} H^{*}(A)$ which is a predecessor for these obstructions.
\end{abstract}

\section{INTRODUCTION}

This paper begins with a study of the following question. Let $k$ be a field and let $G$ be a finite group. Given a graded module $X$ over the Tate cohomology ring $\hat{H}^{*}(G, k)$, how do we decide whether there exists a $k G$-module $M$ such that $\hat{H}^{*}(G, M) \cong X$ ?

We do not give a complete answer to this question. However, we do answer the weaker question of how to decide whether there exists a $k G$-module $M$ such that $X$ is isomorphic to a direct summand of the graded $\hat{H}^{*}(G, k)$-module $\hat{H}^{*}(G, M)$.

Theorem 1.1. Let $k$ be a field, $G$ a finite group, and let $\hat{H}^{*}(G)$ denote the graded Tate cohomology algebra of $G$ over $k$. Then there exists a canonical element in Hochschild cohomology of $\hat{H}^{*}(G)$

$$
\gamma_{G} \in H H^{3,-1} \hat{H}^{*}(G),
$$

such that for any graded $\hat{H}^{*}(G)$-module $X$, the following are equivalent:

(i) The image of $\gamma_{G}$ in $\operatorname{Ext}_{\hat{H}^{*}(G)}^{3,-1}(X, X)$ is zero.

(ii) There exists a $k G$-module $M$ such that $X$ is a direct summand of the graded $\hat{H}^{*}(G)$-module $\hat{H}^{*}(G, M)$.

Theorem 1.1 is really a lot more general; finite groups are irrelevant, except inasmuch as they allow us to form complete resolutions. Complete resolutions are defined in general for modules over a self-injective algebra, and this is the context in which we prove our result; see Theorem 6.9. For further directions of generalization, see Remark 6.11

Received by the editors April 5, 2002 and, in revised form, April 25, 2003.

2000 Mathematics Subject Classification. Primary 20J06; Secondary 16E40, 16E45, 55S35.

The first author was partly supported by NSF grant DMS-9988110. 
The program for proving Theorem 1.1 breaks up into two parts:

(A) There is a general obstruction theory for the realization question in triangulated categories. It produces classes of the form $\kappa(f) \in \operatorname{Ext}_{E}^{3,-1}(X, Y)$ which are the obstructions for a homomorphism $f: X \rightarrow Y$ of abstract $E$-modules to factor through a realizable module.

(B) In certain cases, the realizability obstructions $\kappa(f)$ are equal to the image of some Hochschild cohomology class $\gamma \in H H^{3,-1} E$ via the relation $\kappa(f)=$ $f \cup \gamma$. The global class $\gamma$ depends on a 'model' for the given triangulated category. We provide such a global class in two cases, namely for the derived category of a differential graded algebra and the stable module category of a self-injective algebra (for example, the group algebra of a finite group).

In more detail, the plan of the paper is as follows.

(A) Let $\mathcal{T}$ be a triangulated category with infinite sums; two relevant cases are $\mathcal{T}=\mathcal{D}(A)$, the derived category of a differential graded algebra $A$, and $\mathcal{T}=$ StMod- $L$, the stable module category of a self-injective $k$-algebra $L$. Let $N$ be a compact object in $\mathcal{T}$, i.e., $\mathcal{T}(N,-)$ commutes with sums. Let $E=\mathcal{T}(N, N)^{*}$ denote the graded endomorphism ring of $N$. If $M$ is any object in $\mathcal{T}$, then $\mathcal{T}(N, M)^{*}$ is a graded right $E$-module by composition. We call an $E$-module realizable if it is isomorphic to $\mathcal{T}(N, M)^{*}$ for some object $M$.

There is a general obstruction theory for deciding which graded $E$-modules are realizable. Given a graded $E$-module $X$, there is an infinite sequence of classes

$$
\kappa_{n} \in \operatorname{Ext}_{E}^{n, 2-n}(X, X)
$$

for $n \geq 3$, such that each successive class $\kappa_{n}$ is defined if the previous one $\kappa_{n-1}$ vanishes, and $\kappa_{n}$ depends on a choice. A graded $E$-module $X$ is realizable if and only if successive choices can be made so that all classes $\kappa_{n}$ are trivial. We do not know of a published source where this obstruction theory is worked out in the generality of triangulated categories, so for the convenience of the reader we review it in Appendix A.

In Sections 2 and 3 we explore the very first in the sequence of realizability obstructions; this class lies in $\operatorname{Ext}_{E}^{3,-1}(X, X)$, it does not depend on any choices, and we denote it $\kappa(X)$. In Section 2 we warm up by considering the important special case of modules over the Tate cohomology algebra of a finite group. In Section 3 we recall how $\kappa(X)$ can be defined in a general triangulated category $\mathcal{T}$; we examine its naturality properties, and we show that $\kappa(X)$ vanishes if and only if $X$ is isomorphic to a direct summand of a realizable $E$-module. More generally, we define a map

$$
\kappa: \operatorname{Hom}_{E}(X, Y) \longrightarrow \operatorname{Ext}_{E}^{3,-1}(X, Y),
$$

natural in both variables, with the following property:

Theorem 1.2. The class $\kappa(f) \in \operatorname{Ext}_{E}^{3,-1}(X, Y)$ vanishes if and only if the homomorphism $f: X \longrightarrow Y$ factors through a realizable E-module.

This theorem is proved as Theorem 3.7. We do not know if the higher obstructions $\kappa_{n}$ to realizing a graded $E$-module also have an explicit interpretation.

(B) The main point of this paper is that in certain cases the realizability obstruction $\kappa(f)$ is equal to the image of some Hochschild cohomology class $\gamma \in H H^{3,-1} E$ via the relation $\kappa(f)=f \cup \gamma$. We do not believe that such a global class exists for 
an arbitrary triangulated category; the class $\gamma$ depends on a 'model' for the given triangulated category.

The relevant Hochschild cohomology class of bidegree $(3,-1)$ is defined for any differential graded algebra $A$ over a field $k$; we refer to this as the canonical class of $A$ and denote it

$$
\gamma_{A} \in H H^{3,-1}\left(H^{*} A\right) .
$$

The ground field $k$ is usually fixed and omitted from the notation, but Hochschild cohomology is always relative to the field $k$. We review the definition of $\gamma_{A}$ in Section 5, For any graded $k$-algebra $\Lambda$ and graded right $\Lambda$-modules $X$ and $Y$, there is a cup product pairing

$$
\cup: \operatorname{Hom}_{\Lambda}(X, Y) \otimes H H^{*, *} \Lambda \longrightarrow \operatorname{Ext}_{\Lambda}^{*, *}(X, Y) ;
$$

see (4.4). In the context where $\mathcal{T}=\mathcal{D}(A)$ is the unbounded derived category of a differential graded algebra $A$ and $N$ is the free $\operatorname{dg}$ - $A$-module of rank one, we have a canonical isomorphism of graded $k$-algebras $\mathcal{D}(A)(A, A)^{*} \cong H^{*} A$. The obstruction $\kappa(f)$ of part (A) and the canonical class $\gamma_{A}$ are linked via the cup product pairing for $\Lambda=H^{*} A$, the cohomology algebra of $A$ :

Theorem 1.3. Let $A$ be a differential graded $k$-algebra and let $\gamma_{A}$ be its canonical Hochschild cohomology class. For a morphism $f: X \longrightarrow Y$ of graded $H^{*} A$-modules, the realizability obstruction $\kappa(f) \in \operatorname{Ext}_{H^{*}(A)}^{3,-1}(X, Y)$, constructed from the derived category $\mathcal{D}(A)$ of differential graded $A$-modules, is equal to the class $f \cup \gamma_{A}$.

This theorem reappears as Theorem [6.2] and we prove it in Section 9 The second context in which there is a universal predecessor to the obstructions $\kappa(f)$ is $\mathcal{T}=$ StMod- $L$, the stable module category of a self-injective $k$-algebra $L$ (for example $L=k G$, the group algebra of a finite group $G$ ). We reduce the case of stable module categories to the previous theorem using the endomorphism dga of a complete resolution; see Section 6.4. The result is stated as Theorem 6.9 which has Theorem 1.1 as a special case.

In Sections 7 and 8 we work out some examples involving Tate cohomology of finite groups. For finite cyclic groups of prime power order, it turns out that the class $\gamma$ is equal to zero except in just one case, namely that of the cyclic group of order 3 in characteristic 3 . In this case, the element $\gamma$ picks up the nontrivial Massey triple products of nilpotent elements of cohomology. We also discuss the Klein four group in characteristic two, where elements of Tate cohomology of negative degree have to be used in order to show that $\gamma$ is nonzero.

In Section 8, we use transfer and restriction to show how to reduce from a general finite group $G$ to a Sylow $p$-subgroup $P$, where $p$ is the characteristic of $k$. Theorem 8.3 gives the explicit formula $\gamma_{G}=\operatorname{tr}_{*}\left(\operatorname{res}^{*}\left(\gamma_{P}\right)\right) /[G: P]$. We then use this to discuss groups with cyclic Sylow $p$-subgroups.

The Hochschild cohomology class $\gamma_{A}$ is not at all new, and it has come up in several other contexts before. The class is the first piece of the $A_{\infty}$-structure on the cohomology $H^{*} A$. The concept of an $A_{\infty}$-algebra was introduced by Stasheff [38] while studying higher order associativity conditions on H-spaces; Kadeishvili showed that the cohomology of any differential graded algebra $A$ has an $A_{\infty^{-}}$ structure, unique up to (non-canonical) isomorphism, which is $A_{\infty}$-quasi-isomorphic to $A$ [25]. Hence the entire $A_{\infty}$-structure on $H^{*} A$ recovers the quasi-isomorphism type of $A$. Kadeishvili also showed that specifying an $A_{3}$-structure on an associative 
graded $k$-algebra $\Lambda$ amounts to choosing a Hochschild $(3,-1)$-cocycle of $\Lambda$, and that the isomorphisms classes of such $A_{3}$-structures are in bijective correspondence with classes in $H H^{3,-1} \Lambda$ [26]. The class $\gamma_{A}$ contains the information about Massey triple products in the cohomology of the dga $A$; see Remark 5.10. For an introduction to $A_{\infty}$-algebras we recommend [29].

Berrick and Davydov [1] use the canonical Hochschild class to obtain multiplicative information about the cohomology of the total space in a spherical fibration. A generalization of the class $\gamma_{A}$ has been studied by Baues and coauthors [2], [3], [4]; their class, called the 'universal Toda bracket', lies in the $(3,-1)$-cohomology group of an appropriate homotopy category.

For simplicity we restrict our attention to algebras over a field $k$. However, the theory can be generalized to differential graded algebras $A$ over a commutative ground ring $k$, as long as the cohomology algebra $H^{*} A$ is dimensionwise projective over $k$. In that situation the construction of the class $\gamma_{A}$ carries over and Theorem 1.3 holds as stated.

1.4. Notations and conventions. Throughout the paper, $k$ is a fixed ground field, and $\otimes$ is short for the tensor product over $k$. Whenever convenient, we shall write a tensor product $\lambda_{1} \otimes \cdots \otimes \lambda_{n}$ as an $n$-tuple $\left(\lambda_{1}, \ldots, \lambda_{n}\right)$.

When we talk about modules, we always mean right modules. We denote by Mod- $L$ the category of all (not necessarily finitely generated) right modules over a $k$ algebra $L$. Suppose that $L$ is a Frobenius algebra, i.e., that the classes of projective and injective $L$-modules coincide. We write StMod- $L$ for the stable category of $L$ modules. The objects in this category are the same as in Mod- $L$, but the morphisms are given by

$$
\underline{\operatorname{Hom}}_{L}(N, M)=\operatorname{Hom}_{L}(N, M) / \operatorname{PHom}_{L}(N, M),
$$

where $\operatorname{PHom}_{L}(N, M)$ is the $k$-linear subspace consisting of homomorphisms which factor through some projective module. As usual we denote by $\Omega M$ the syzygy module of an $L$-module $M$, i.e., the kernel of an epimorphism $P \rightarrow M$ with projective source; $\Omega$ becomes a functor on the level of the stable module category. The category StMod- $L$ is a triangulated category, with shift given by the inverse of $\Omega$ and where the triangles come from short exact sequences in Mod- $L$ (see for example 24], or [13, Thm. 5.6] in the case of group algebras).

If $V$ is a $\mathbb{Z}$-graded $k$-module, we write $V[n]$ for the graded $k$-module with $V[n]^{i}=$ $V^{n+i}$. If $V$ supports a differential $d: V^{i} \longrightarrow V^{i+1}$, then the differential on $V[n]$ is given by $(-1)^{n} d$. If $V$ and $W$ are graded $k$-modules, we write

$$
\operatorname{Hom}_{k}^{n}(V, W)=\operatorname{Hom}_{k}(V, W[n])=\prod_{j} \operatorname{Hom}_{k}\left(V^{j}, W^{n+j}\right)
$$

for the space of graded $k$-module maps of degree $n$, so that $\operatorname{Hom}_{k}^{*}(V, W)$ is also a graded $k$-module. If $V$ and $W$ support a differential, then the differential

$$
d: \operatorname{Hom}_{k}^{n}(V, W) \longrightarrow \operatorname{Hom}_{k}^{n+1}(V, W)
$$

is given by

$$
d(f)(v)=d(f(v))-(-1)^{n} f(d(v)) .
$$

Similarly, we write $(V \otimes W)^{n}$ for $\bigoplus_{i+j=n} V^{i} \otimes W^{j}$. So $V \otimes W$ is again a graded $k$-module. If $V$ and $W$ support differentials, then the differential

$$
d:(V \otimes W)^{n} \longrightarrow(V \otimes W)^{n+1}
$$


is given by

$$
d(v \otimes w)=d(v) \otimes w+(-1)^{|v|} v \otimes d(w) .
$$

Cohomology of graded modules over a graded algebra is bigraded; the first index gives the cohomological degree and the second gives the internal degree. For example, given graded modules $V$ and $W$ over a graded algebra $\Lambda$,

$$
\operatorname{Ext}_{\Lambda}^{s, t}(V, W)=\operatorname{Ext}_{\Lambda}^{s}(V, W[t])
$$

So an element in $\operatorname{Ext}_{\Lambda}^{3,-1}(V, W)$ is represented by an exact sequence of graded $\Lambda$-modules

$$
0 \longrightarrow W[-1] \longrightarrow X_{2} \longrightarrow X_{1} \longrightarrow X_{0} \longrightarrow V \longrightarrow 0
$$

In a triangulated category $\mathcal{T}$, we write $\mathcal{T}(N, M)^{n}=\mathcal{T}(N, M[n])$, where $N[n]$ is the $n$-fold shift of $N$. Then $\mathcal{T}(N, N)^{*}$ is a graded ring and $\mathcal{T}(N, M)^{*}$ forms a graded right $\mathcal{T}(N, N)^{*}$-module under composition. For general background on derived and triangulated categories, see SGA4 $\frac{1}{2}$ [18] (Appendix by Verdier), Gelfand and Manin [21], Verdier [40, and Weibel [41]. For background on unbounded complexes, see Spaltenstein [37.

\section{A Realizability obstruction in STABle module CATEGories}

We fix a field $k$ and a finite group $G$, and we write $\hat{H}^{*}(G)$ for the Tate cohomology of $G$ with coefficients in $k$. In this section, given a graded $\hat{H}^{*}(G)$-module $X$, we define an obstruction

$$
\kappa(X) \in \operatorname{Ext}_{\hat{H}^{*}(G)}^{3,-1}(X, X)=\operatorname{Ext}_{\hat{H}^{*}(G)}^{3}(X, X[-1]) .
$$

We prove in Theorem [2.3 that $\kappa(X)=0$ if and only if $X$ is isomorphic to a direct summand of an $\hat{H}^{*}(G)$-module of the form $\hat{H}^{*}(G, M)$, for some $k G$-module $M$. In Section 6.4 we define the canonical Hochschild cohomology class

$$
\gamma_{G} \in H H^{3,-1} \hat{H}^{*}(G)
$$

and show that $\kappa(X)$ is the image of $\gamma_{G}$ under the natural map from Hochschild cohomology to Ext.

Construction 2.1. Let $X$ be a graded $\hat{H}^{*}(G)$-module, and let

$$
F_{1} \stackrel{\rho}{\rightarrow} F_{0} \longrightarrow X \longrightarrow 0
$$

be the beginning of a free resolution of $X$ over $\hat{H}^{*}(G)$. In other words, each of $F_{0}$ and $F_{1}$ is a direct sum of copies of $\hat{H}^{*}(G)$, with degree shifts as necessary to hit generators and relations for $X$.

The Tate cohomology functor $\hat{H}^{*}(G,-)$ distributes over direct sums. Since

$$
\hat{H}^{*}\left(G, \Omega^{n} k\right) \cong \hat{H}^{*}(G)[-n],
$$

we can find $k G$-modules $R_{0}$ and $R_{1}$, each a direct sum of modules of the form $\Omega^{n} k$, $n \in \mathbb{Z}$, such that $\hat{H}^{*}\left(G, R_{0}\right) \cong F_{0}$ and $\hat{H}^{*}\left(G, R_{1}\right) \cong F_{1}$. Furthermore, there is a map $\alpha: R_{1} \longrightarrow R_{0}$ of $k G$-modules such that

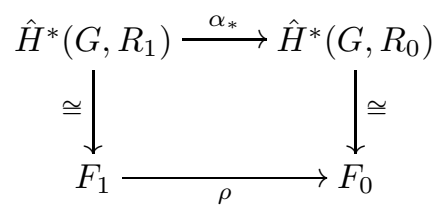


commutes. By adding an injective (= projective) module to $R_{0}$ if necessary, we can assume that $\alpha$ is injective. We let $B$ denote the cokernel of $\alpha: R_{1} \longrightarrow R_{0}$. We get a long exact sequence in Tate cohomology

$$
\cdots \longrightarrow \hat{H}^{*}(G, B)[-1] \longrightarrow \hat{H}^{*}\left(G, R_{1}\right) \stackrel{\alpha_{*}}{\longrightarrow} \hat{H}^{*}\left(G, R_{0}\right) \longrightarrow \hat{H}^{*}(G, B) \longrightarrow \cdots .
$$

Since $X$ is identified with the cokernel of the map $\alpha_{*}$, a piece of this long exact sequence is the exact sequence of $\hat{H}^{*}(G)$-modules

$$
0 \longrightarrow X[-1] \longrightarrow \hat{H}^{*}(G, B)[-1] \longrightarrow F_{1} \stackrel{\rho}{\rightarrow} F_{0} \longrightarrow X \longrightarrow 0 \text {. }
$$

We define $\kappa(X)$ as the Yoneda-class of this exact sequence in $\operatorname{Ext}_{\hat{H}^{*}(G)}^{3,-1}(X, X)$. We will show in Proposition 3.4 (in the more general context of triangulated categories) that the class $\kappa(X)$ is independent of the choice of resolution $\rho: F_{1} \rightarrow F_{0}$ and the choice of the map $\alpha$ realizing it.

Theorem 2.3. The following are equivalent:

(i) $\kappa(X)=0$ in $\operatorname{Ext}_{\hat{H}^{*}(G)}^{3,-1}(X, X)$.

(ii) The $\hat{H}^{*}(G)$-module $X$ is isomorphic to a direct summand of $\hat{H}^{*}(G, M)$ for some $k G$-module $M$.

Proof. In the exact sequence (2.2) which represents $\kappa(X)$, the modules $F_{1}$ and $F_{0}$ are projective. So the Yoneda class of the sequence is trivial if and only if the monomorphism $X \longrightarrow \hat{H}^{*}(G, B)$ has a left inverse. So if (i) holds, then $X$ is a direct summand of the realizable module $\hat{H}^{*}(G, B)$.

Conversely, since the construction of $\kappa(X)$ is additive in $X$, we may assume that $X=\hat{H}^{*}(G, M)$. Then the map $F_{0} \longrightarrow X$ is realized by a map $\beta: R_{0} \longrightarrow M$ whose composite with $\alpha: R_{1} \longrightarrow R_{0}$ is trivial in Tate cohomology. Since $R_{1}$ is a sum of modules of the form $\Omega^{n} k$, the composite map $\beta \alpha: R_{1} \longrightarrow M$ is zero in the stable category of $k G$-modules. So after subtracting a map factoring through an injective (= projective) module, if necessary, $\beta: R_{0} \longrightarrow M$ factors through the cokernel of $\alpha$ and gives a map $\bar{\beta}: B \rightarrow M$. The induced map $\bar{\beta}_{*}: \hat{H}^{*}(G, B) \longrightarrow \hat{H}^{*}(G, M)=X$ is a retraction to the monomorphism $X \longrightarrow \hat{H}^{*}(G, B)$, so the exact sequence (2.2) represents the trivial element in $\operatorname{Ext}_{\hat{H}^{*}(G)}^{3,-1}(X, X)$.

Theorem 2.3 is a special case of a similar theorem which works in a more general context of triangulated categories, see Theorem 3.7 below.

Example 2.4 (Cyclic modules over cohomology algebras). Let $\zeta \in \hat{H}^{n}(G)$ be a non-trivial Tate cohomology class of dimension $n$. Then $\zeta$ is represented by a surjective $k G$-linear map $\hat{\zeta}: \Omega^{n} k \rightarrow k$. The kernel of $\hat{\zeta}$ is denoted $L_{\zeta}$; it is independent of the choice of representative $\hat{\zeta}$ up to isomorphism in the stable module category. The role of the module $B$ in the construction of $\kappa(X)$ is now played by $\Omega^{-1} L_{\zeta}$. Applying Tate cohomology to the short exact sequence

$$
0 \longrightarrow L_{\zeta} \stackrel{\iota}{\rightarrow} \Omega^{n} k \stackrel{\hat{\zeta}}{\rightarrow} k \longrightarrow 0
$$

gives an exact sequence of Tate cohomology modules

$$
\begin{aligned}
0 \longrightarrow \hat{H}^{*}(G) /(\zeta)[-1] & \longrightarrow \hat{H}^{*}\left(G, L_{\zeta}\right) \stackrel{\iota_{*}}{\longrightarrow} \hat{H}^{*}(G)[-n] \\
\stackrel{. \zeta}{\longrightarrow} \hat{H}^{*}(G) & \longrightarrow \hat{H}^{*}(G) /(\zeta) \longrightarrow 0
\end{aligned}
$$


which represents the obstruction class of the cyclic module $\hat{H}^{*}(G) /(\zeta)$. Hence a cyclic Tate cohomology module $\hat{H}^{*}(G) /(\zeta)$ is a summand of a realizable module if and only if $\hat{H}^{*}(G) /(\zeta)[-1]$ splits off $\hat{H}^{*}\left(G, L_{\zeta}\right)$. If $\zeta$ is not a zero divisor, then $\iota_{*}$ is trivial, the sequence splits, and $\hat{H}^{*}(G) /(\zeta)$ is actually realizable, namely by $\Omega^{-1} L_{\zeta}$. For an explicit example where the Tate cohomology of $L_{\zeta}$ does not split off a shifted copy of $\hat{H}^{*}(G) /(\zeta)$, one may take $G=C_{3}$, the cyclic group of order 3 over a field $k$ of characteristic 3 . For $\zeta$ we take any non-trivial 1-dimensional class in $\hat{H}^{1}\left(C_{3}, k\right)$; this example is discussed in more detail in 7.6

\section{A Realizability obstruCtion in triangulated CATEGORIES}

In this section, we generalize the obstruction $\kappa(X)$ of Section 2 to triangulated categories and investigate its naturality properties. Let $\mathcal{T}$ be a triangulated category with arbitrary direct sums and let $N$ be a compact object in $\mathcal{T}$. In other words, $\mathcal{T}(N,-)$ commutes with direct sums. Denote by $E=\mathcal{T}(N, N)^{*}$ the graded endomorphism algebra of $N$ in $\mathcal{T}$. If $M$ is any object in $\mathcal{T}$ then $\mathcal{T}(N, M)^{*}$ is a graded $E$-module by composition. We call an E-module realizable if it is isomorphic to $\mathcal{T}(N, M)^{*}$ for some object $M$.

Given two graded right $E$-modules $X$ and $Y$, we construct an additive map

$$
\kappa: \operatorname{Hom}_{E}(X, Y) \longrightarrow \operatorname{Ext}_{E}^{3,-1}(X, Y)
$$

which measures whether a morphism of graded $E$-modules factors through a realizable module. The map $\kappa$ is actually a natural transformation of functors of two variables, see Remark 3.9 (i).

Definition 3.1. A $\mathcal{T}$-presentation of an $E$-module $X$ consists of a distinguished triangle

$$
B[-1] \stackrel{\delta}{\rightarrow} R_{1} \stackrel{\alpha}{\rightarrow} R_{0} \stackrel{\pi}{\rightarrow} B
$$

in $\mathcal{T}$ together with an epimorphism of graded $E$-modules $\varepsilon: \mathcal{T}\left(N, R_{0}\right)^{*} \rightarrow X$ such that the sequence

$$
\mathcal{T}\left(N, R_{1}\right)^{*} \stackrel{\alpha_{*}}{\longrightarrow} \mathcal{T}\left(N, R_{0}\right)^{*} \stackrel{\varepsilon}{\rightarrow} X \longrightarrow 0
$$

is exact. We say that an object $R$ of $\mathcal{T}$ is $N$-free if $R$ is isomorphic to a direct sum of shifted copies of $N$. The point of this definition is that whenever $R$ is $N$-free and $M \in \mathcal{T}$ is arbitrary, then the map

$$
\mathcal{T}(N,-)^{*}: \mathcal{T}(R, M) \longrightarrow \operatorname{Hom}_{E}\left(\mathcal{T}(N, R)^{*}, \mathcal{T}(N, M)^{*}\right)
$$

is an isomorphism; in other words: $E$-module homomorphisms from $\mathcal{T}(N, R)^{*}$ to $\mathcal{T}(N, M)^{*}$ are uniquely realizable (this uses the compactness of $N$ ). We call a $\mathcal{T}$ presentation $N$-special if $R_{0}$ and $R_{1}$ are $N$-free; if $N=k$, we drop it from the notation and just refer to a special $\mathcal{T}$-presentation.

Given a $\mathcal{T}$-presentation

$$
\left(B[-1] \stackrel{\delta}{\rightarrow} R_{1} \stackrel{\alpha}{\rightarrow} R_{0} \stackrel{\pi}{\rightarrow} B, \varepsilon: \mathcal{T}\left(N, R_{0}\right)^{*} \rightarrow X\right)
$$

we can apply the functor $\mathcal{T}(N,-)^{*}$ to the triangle and get an exact sequence of graded $E$-modules

$$
0 \rightarrow X[-1] \stackrel{\eta[-1]}{\longrightarrow} \mathcal{T}(N, B)^{*}[-1] \stackrel{\delta_{*}}{\longrightarrow} \mathcal{T}\left(N, R_{1}\right)^{*} \stackrel{\alpha_{*}}{\longrightarrow} \mathcal{T}\left(N, R_{0}\right)^{*} \stackrel{\varepsilon}{\rightarrow} X \rightarrow 0
$$


the monomorphism $\eta: X \rightarrow \mathcal{T}(N, B)^{*}$ is determined by $\eta \circ \varepsilon=\pi_{*}$. We refer to this sequence as the associated extension of the $\mathcal{T}$-presentation.

Proposition 3.4. Let $X$ and $Y$ be graded E-modules.

(i) There exists an $N$-special $\mathcal{T}$-presentation of $X$.

(ii) The Yoneda-class in $\operatorname{Ext}_{E}^{3,-1}(X, X)$ of the associated extension is independent of the $\mathcal{T}$-presentation of $X$; we denote this class by $\kappa(X)$.

(iii) Let $\eta: X \rightarrow \mathcal{T}(N, B)^{*}$ be the monomorphism associated to an $N$-special $\mathcal{T}$-presentation of $X$. Then for any object $M$ of $\mathcal{T}$ and any homomorphism of graded E-modules $f: X \longrightarrow \mathcal{T}(N, M)^{*}$ there exists a map $\beta: B \longrightarrow M$ in $\mathcal{T}$ such that $f=\beta_{*} \circ \eta$.

(iv) (Additivity) We have

$$
\kappa(X \oplus Y)=\kappa(X) \oplus \kappa(Y) \quad \text { in } \quad \operatorname{Ext}_{E}^{3,-1}(X \oplus Y, X \oplus Y) .
$$

(v) (Naturality) For every $n \geq 0$ and every class $\beta \in \operatorname{Ext}^{n}(X, Y)$ we have

$$
\beta \circ \kappa(X)=(-1)^{n} \kappa(Y) \circ \beta
$$

in the group $\operatorname{Ext}_{E}^{n+3,-1}(X, Y)$, where the circle denotes Yoneda composition. We denote this class by $\kappa(\beta)$.

Proof. (i) We start by choosing the beginning of a free resolution of a given $E$ module $X$

$$
F_{1} \stackrel{\rho}{\rightarrow} F_{0} \stackrel{\varepsilon}{\rightarrow} X \longrightarrow 0 .
$$

Every free $E$-module can be realized in $\mathcal{T}$ by a sum of suitable shifts of the object $N$, i.e., by an $N$-free object. Moreover, homomorphisms between $N$-free objects can be uniquely realized in $\mathcal{T}$. So we can choose a morphism $\alpha: R_{1} \longrightarrow R_{0}$ between $N$-free objects in $\mathcal{T}$ which induces $\rho: F_{1} \rightarrow F_{0}$ after applying the functor $\mathcal{T}(N,-)^{*}$. Then we complete $\alpha$ to a distinguished triangle.

(ii) Let

$$
\left(B[-1] \stackrel{\delta}{\rightarrow} R_{1} \stackrel{\alpha}{\rightarrow} R_{0} \stackrel{\pi}{\rightarrow} B, \varepsilon: \mathcal{T}\left(N, R_{0}\right)^{*} \rightarrow X\right)
$$

be a fixed $N$-special $\mathcal{T}$-presentation of $X$, and suppose that

$$
\left(B^{\prime}[-1] \stackrel{\delta^{\prime}}{\longrightarrow} R_{1}^{\prime} \stackrel{\alpha}{\rightarrow} R_{0}^{\prime} \stackrel{\pi^{\prime}}{\longrightarrow} B^{\prime}, \varepsilon^{\prime}: \mathcal{T}\left(N, R_{0}^{\prime}\right)^{*} \rightarrow X\right)
$$

is another $\mathcal{T}$-presentation of $X$. In the commutative diagram of $E$-modules

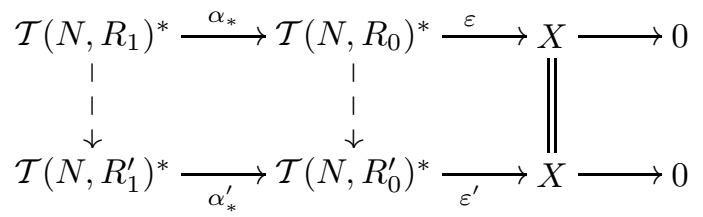

with exact rows, the vertical arrows are constructed using the standard lifting argument. Since $R_{1}$ and $R_{0}$ are $N$-free, the left square can be uniquely realized in the triangulated category $\mathcal{T}$, and can then be completed (non-canonically) to a map of triangles

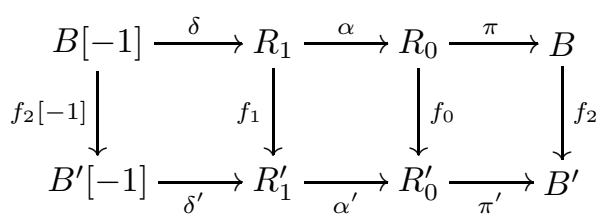


This in turn gives rise to a map of extensions

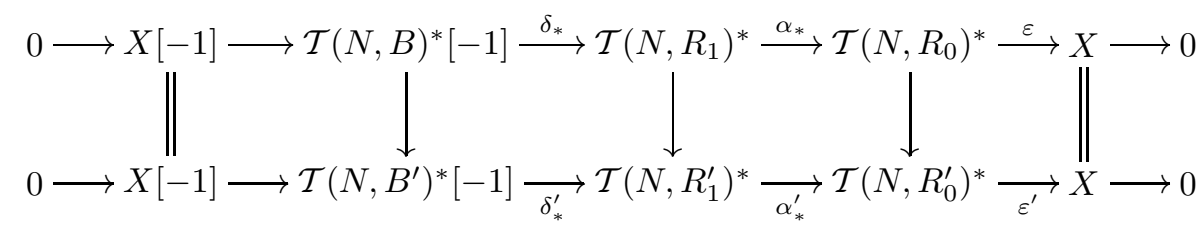

which proves that the associated classes in $\operatorname{Ext}_{E}^{3,-1}(X, X)$ are equal.

(iii) Since $R_{0}$ is $N$-free, the composite map

$$
\mathcal{T}\left(N, R_{0}\right)^{*} \stackrel{\varepsilon}{\rightarrow} X \stackrel{f}{\rightarrow} \mathcal{T}(N, M)^{*}
$$

is realized by a unique map $h: R_{0} \longrightarrow M$ in $\mathcal{T}$. The composite $h \alpha: R_{1} \longrightarrow M$ induces the zero map $\mathcal{T}(N,-)^{*}$, so it is trivial in $\mathcal{T}$ since $R_{1}$ is $N$-free. Hence $h$ can be extended over a map $\beta: B \rightarrow M$ such that $\beta \pi=h$. The formula $\beta_{*} \circ \eta=f$ holds since it holds after composition with the epimorphism $\varepsilon$.

(iv) The functor $\mathcal{T}(N,-)^{*}$ commutes with direct sums and the direct sum of two distinguished triangles is again a distinguished triangle. So we obtain a $\mathcal{T}$ presentation of $X \oplus Y$ as the direct sum of $\mathcal{T}$-presentations of $X$ and $Y$. With these choices, the associated extension of the direct sum $\mathcal{T}$-presentation of $X \oplus Y$ is the direct sum of the associated extensions which represent $\kappa(X)$ and $\kappa(Y)$.

(v) The case $n=0$ - i.e., naturality for homomorphisms of graded $E$-modules - is very similar to part (ii) and is therefore omitted. For $n \geq 2$, every class in $\operatorname{Ext}_{E}^{n}(X, Y)$ is a Yoneda product of Ext-classes of dimension 1, involving possibly different modules, so it remains to treat the case $n=1$. Let

$$
\left(B[-1] \stackrel{\delta}{\rightarrow} R_{1} \stackrel{\alpha}{\rightarrow} R_{0} \stackrel{\pi}{\rightarrow} B, \varepsilon: \mathcal{T}\left(N, R_{0}\right)^{*} \rightarrow X\right)
$$

be an $N$-special $\mathcal{T}$-presentation of $X$, and let $\Omega X$ denote the kernel of the epimorphism $\varepsilon$. Denote by $\omega \in \operatorname{Ext}_{E}^{1}(X, \Omega X)$ the class of the extension

$$
0 \longrightarrow \Omega X \longrightarrow \mathcal{T}\left(N, R_{0}\right)^{*} \stackrel{\varepsilon}{\rightarrow} X \longrightarrow 0 .
$$

Since $\mathcal{T}\left(N, R_{0}\right)^{*}$ is a projective $E$-module, an arbitrary class $\beta \in \operatorname{Ext}_{E}^{1}(X, Y)$ is of the form $\beta=f \circ \omega$ for some homomorphism $f: \Omega X \rightarrow Y$. Naturality of $\kappa$ for homomorphisms is already taken care of, so we are reduced to checking naturality for the special one-dimensional Ext-class $\omega$.

Denote by $\lambda \in \operatorname{Ext}_{E}^{2,-1}(\Omega X, X)$ the class of the extension

$$
0 \longrightarrow X[-1] \longrightarrow \mathcal{T}(N, B)^{*}[-1] \stackrel{\delta_{*}}{\longrightarrow} \mathcal{T}\left(N, R_{1}\right)^{*} \stackrel{\bar{\varepsilon}}{\rightarrow} \Omega X \longrightarrow 0 .
$$

Splicing these two sequences (3.5) and (3.6) gives the associated extension of the chosen $\mathcal{T}$-presentation of $X$, so we have $\kappa(X)=\lambda \circ \omega$.

Rotating the triangle gives a $\mathcal{T}$-presentation of $\Omega X$ :

$$
\left(R_{0}[-1] \stackrel{-\pi[-1]}{\longrightarrow} B[-1] \stackrel{\delta}{\rightarrow} R_{1} \stackrel{\alpha}{\rightarrow} R_{0}, \bar{\varepsilon}: \mathcal{T}\left(N, R_{1}\right)^{*} \rightarrow \Omega X\right)
$$

(note the sign change which occurs when rotating a distinguished triangle). The extension associated to this $\mathcal{T}$-presentation of $\Omega X$ has the form

$$
\begin{aligned}
0 \longrightarrow \Omega X[-1] & \longrightarrow \mathcal{T}\left(N, R_{0}\right)^{*}[-1] \stackrel{-\pi_{*}[-1]}{\longrightarrow} \\
& \mathcal{T}(N, B)^{*}[-1] \stackrel{\delta_{*}}{\longrightarrow} \mathcal{T}\left(N, R_{1}\right)^{*} \stackrel{\bar{\varepsilon}}{\rightarrow} \Omega X \longrightarrow 0 .
\end{aligned}
$$


This is the splice of the sequences (3.5) and (3.6) in the other order, except for the sign in front of the map $\pi_{*}[-1]$. So we have $\kappa(\Omega X)=-\omega \circ \lambda$. Combining this with the previous decomposition of $\kappa(X)$ we obtain

$$
\omega \circ \kappa(X)=\omega \circ \lambda \circ \omega=-\kappa(\Omega X) \circ \omega .
$$

Theorem 3.7. Let $\mathcal{T}$ be a triangulated category with infinite sums, $N$ a compact object in $\mathcal{T}$ and $E=\mathcal{T}(N, N)^{*}$ the graded endomorphism ring of $N$. Let

$$
\kappa: \operatorname{Hom}_{E}(X, Y) \longrightarrow \operatorname{Ext}_{E}^{3,-1}(X, Y)
$$

be the map defined in part (v) of Proposition 3.4 (for the case $n=0$ ). Then the kernel of $\kappa$ consists precisely of those morphisms of graded E-modules which factor through an E-module of the form $\mathcal{T}(N, M)^{*}$ for some object $M \in \mathcal{T}$. In particular, an E-module $X$ is isomorphic to a direct summand of a module of the form $\mathcal{T}(N, M)^{*}$ if and only if $\kappa(X)=0$.

Proof. Choose an $N$-special $\mathcal{T}$-presentation

$$
\left(B[-1] \stackrel{\delta}{\rightarrow} R_{1} \stackrel{\alpha}{\rightarrow} R_{0} \stackrel{\pi}{\rightarrow} B, \varepsilon: \mathcal{T}\left(N, R_{0}\right)^{*} \rightarrow X\right)
$$

of $X$ and let $f: X \longrightarrow Y$ be a homomorphism of $E$-modules. Since the $E$-modules $\mathcal{T}\left(N, R_{1}\right)^{*}$ and $\mathcal{T}\left(N, R_{0}\right)^{*}$ are free, the extension $f \circ \kappa(X)$ represents the trivial class in $\operatorname{Ext}_{E}^{3,-1}(X, Y)$ if and only if the map $f$ can be extended over the monomorphism $\eta: X \longrightarrow \mathcal{T}(N, B)^{*}$. So if $\kappa(f)=0$, then $f$ factors through a realizable module, namely $\mathcal{T}(N, B)^{*}$.

For the converse suppose that there is an object $M \in \mathcal{T}$ and a factorization

$$
X \stackrel{i}{\rightarrow} \mathcal{T}(N, M)^{*} \stackrel{j}{\rightarrow} Y
$$

of $f$. Part (iii) of Proposition 3.4 shows that $i$ extends over the monomorphism $\eta: X \rightarrow \mathcal{T}(N, B)^{*}$. Hence the extension $i \circ \kappa(X)$ represents the trivial element in $\operatorname{Ext}_{E}^{3,-1}\left(X, \mathcal{T}(N, M)^{*}\right)$ and we conclude that $\kappa(f)=j \circ(i \circ \kappa(X))$ is trivial.

Corollary 3.8. Suppose that the graded ring $E$ is sparse in the following sense: there exists a number $N \geq 2$ such that $E$ is concentrated in dimensions divisible by $N$. Then every graded E-module is a direct summand of a realizable module.

Proof. Suppose that $E$ is sparse with period $N$, and let $X$ be any graded $E$-module. For every $i \in \mathbb{Z}$, the elements of $X$ in dimensions congruent to $i$ modulo $N$ form an $E$-submodule $X^{(i)}$ of $X$. The module $X^{(i)}$ is concentrated in dimensions congruent to $i$ modulo $N$, whereas $X^{(i)}[-1]$ is concentrated in dimensions congruent to $i+1$ modulo $N$. Since $N \geq 2$, every extension, of any length, of $X^{(i)}$ by $X^{(i)}[-1]$ is trivial. So the class $\kappa\left(X^{(i)}\right)$ is trivial. Since $X$ is the direct sum of the $E$-modules $X^{(i)}$ for $i=1, \ldots, N$, we get $\kappa(X)=0$ by additivity.

Remarks 3.9. (i) It is a formal consequence of part (v) of Proposition 3.4 that the map

$$
\kappa: \operatorname{Ext}_{E}^{n}(X, Y) \longrightarrow \operatorname{Ext}_{E}^{n+3}(X, Y[-1])
$$

is a natural transformation of functors in two variables, i.e., for $\beta \in \operatorname{Ext}_{E}^{n}(X, Y)$ and $\gamma \in \operatorname{Ext}_{E}^{m}(Y, Z)$ we have

$$
\gamma \circ \kappa(\beta)=\kappa(\gamma \circ \beta)=\kappa(\gamma) \circ \beta \text { in } \operatorname{Ext}_{E}^{m+n+3}(X, Z[-1]) .
$$

(ii) Let $X$ be a graded $E$-module and denote by $\Omega X$ the kernel of any epimorphism $P \rightarrow X$ from a projective $E$-module, and denote by $\Omega^{-1} X$ the cokernel of 
any monomorphism $X \rightarrow I$ to an injective $E$-module. We claim that if $\kappa(X)=0$, then the classes $\kappa(\Omega X)$ and $\kappa\left(\Omega^{-1} X\right)$ are also trivial; we expect that the converse does not hold in general. Indeed, assume that $\kappa(X)$ vanishes and denote by $\omega \in \operatorname{Ext}_{E}^{1}(X, \Omega X)$ the class of the extension

$$
0 \longrightarrow \Omega X \longrightarrow P \longrightarrow X \longrightarrow 0 \text {. }
$$

Using Proposition 3.4 (v) we have $\kappa(\Omega X) \circ \omega=-\omega \circ \kappa(X)=0$. Since $P$ is projective, the map

$$
-\circ \omega: \operatorname{Ext}_{E}^{3,-1}(\Omega X, \Omega X) \longrightarrow \operatorname{Ext}_{E}^{4,-1}(X, \Omega X)
$$

is bijective, so $\kappa(\Omega X)=0$ as claimed. The argument for $\Omega^{-1} X$ is similar, but dual.

(iii) Part (iii) of Proposition 3.4 shows that the realizable $E$-modules and the realizable maps form a covariantly finite subcategory of Mod- $E$. Recall from [1] that a subcategory $\mathcal{C}$ of Mod- $E$ is covariantly finite if every object $X$ in $\operatorname{Mod}-E$ admits a left $\mathcal{C}$-approximation. This is by definition a map $X \rightarrow C$ with $C \in \mathcal{C}$ such that for every map $X \rightarrow C^{\prime}$ with $C^{\prime} \in \mathcal{C}$ there exists a (not necessarily unique) map $C \rightarrow C^{\prime}$ in $\mathcal{C}$ making the diagram

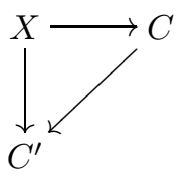

commutative.

(iv) Since injective $E$-modules and morphisms between them are realizable (see Proposition A.4), the realizable $E$-modules and the realizable maps also form a contravariantly finite subcategory of $\operatorname{Mod}-E$, that is, every graded $E$-module $X$ admits a right approximation $\mathcal{T}(N, Q)^{*} \rightarrow X$.

(v) The following line of generalization was suggested to us by Beligiannis. We consider the obstruction $\kappa$ based on a single compact object $N$, but it is also of interest for a family of compact objects. Let $\mathcal{T}$ be a compactly generated triangulated category and denote by $\mathcal{C}$ the full subcategory of compact objects. We view $\mathcal{C}$ as a ring with several objects, and the functor $\mathcal{T} \rightarrow$ Mod- $\mathcal{C}$ which sends $M$ to $\left.\mathcal{T}(-, M)\right|_{\mathcal{C}}$ plays the role of $\mathcal{T}(-, N)^{*}$. The question of which $\mathcal{C}$-modules are realizable is precisely the question of to what extent Brown representability for homology theories holds; see the paper of Christensen, Keller, and Neeman [15] for a recent discussion. The analogue of Theorem 3.7 for families of compact objects provides an answer to this question.

3.10. Relation to $(3,-1)$-periodicity for coherent functors. The natural transformation $\kappa$ is closely related to the $(3,-1)$-periodicity in the graded abelian category of coherent functors from a triangulated category to abelian groups observed by Freyd [20, Sec. 8]. This may be described as follows. If $\mathcal{C}$ is an additive category, a functor $F: \mathcal{C}^{\mathrm{op}} \longrightarrow \mathcal{A} b$ into the category of abelian groups is said to be coherent if there is an exact sequence

$$
\mathcal{C}(-, M) \longrightarrow \mathcal{C}(-, N) \longrightarrow F \longrightarrow 0
$$

(the left natural transformation is then induced by a unique morphism $M \rightarrow N$, by the Yoneda lemma). In particular a coherent functor takes sums to products, and the natural transformations between two coherent functors form a set, and the coherent functors $\mathcal{C}^{\mathrm{op}} \longrightarrow \mathcal{A} b$ form an additive category with cokernels which 
we denote by $\widehat{\mathcal{C}}$. The category $\mathcal{C}$ can be regarded as a subcategory of $\widehat{\mathcal{C}}$ via the Yoneda functor $\mathcal{C} \longrightarrow \widehat{\mathcal{C}}$ which sends $M$ to $\mathcal{C}(-, M)$. In this context, Yoneda's lemma says that the projective objects in $\widehat{\mathcal{C}}$ are the representable functors, namely those isomorphic to objects in the image of the Yoneda functor. Note that if $\mathcal{C}$ has arbitrary coproducts, then $\widehat{\mathcal{C}}$ is a cocomplete category and the Yoneda functor preserves coproducts. Moreover, if $\mathcal{C}$ is triangulated, then $\widehat{\mathcal{C}}$ is abelian.

Theorem 3.11. Let $\mathcal{T}$ be a triangulated category with infinite sums. Let $F$ and $G$ be coherent functors and let $n \geq 0$. Then there is a natural map

$$
\nu: \operatorname{Ext}_{\widehat{\mathcal{T}}}^{n}(F, G) \longrightarrow \operatorname{Ext}_{\widehat{\mathcal{T}}}^{n+3}(F, G[-1])
$$

which is an isomorphism for $n>0$. For $n=0$ the map

$$
\nu: \operatorname{Hom}_{\widehat{\mathcal{T}}}(F, G) \longrightarrow \operatorname{Ext}_{\widehat{\mathcal{T}}}^{3}(F, G[-1])
$$

is an epimorphism with kernel equal to the maps which factor through a representable functor.

Proof. Choose a presentation

$$
\mathcal{T}\left(-, M_{1}\right) \longrightarrow \mathcal{T}\left(-, M_{0}\right) \longrightarrow F \longrightarrow 0 .
$$

Completing $M_{1} \longrightarrow M_{0}$ to a triangle $M_{0}[-1] \longrightarrow M_{2} \longrightarrow M_{1} \longrightarrow M_{0}$ in $\mathcal{T}$ gives a projective resolution $P_{*}$

$$
\begin{aligned}
\cdots \longrightarrow \mathcal{T}\left(-, M_{2}[-1]\right) \longrightarrow \mathcal{T}\left(-, M_{1}[-1]\right) & \longrightarrow \mathcal{T}\left(-, M_{0}[-1]\right) \\
& \longrightarrow \mathcal{T}\left(-, M_{2}\right) \longrightarrow \mathcal{T}\left(-, M_{1}\right) \longrightarrow \mathcal{T}\left(-, M_{0}\right)
\end{aligned}
$$

of $F$ in $\widehat{\mathcal{T}}$. Notice that the shift functor of the triangulated structure induces a map of complexes from $\operatorname{Hom}_{\widehat{\mathcal{T}}}\left(P_{*}, G\right)$ to $\operatorname{Hom}_{\widehat{\mathcal{T}}}\left(P_{*}, G[-1]\right)$ which shifts degrees by three, and which is an isomorphism in positive degrees.

Now let $N$ be a compact object in $\mathcal{T}$; as before, let $E=\mathcal{T}(N, N)^{*}$ denote the graded endomorphism ring of $N$. Evaluation at the shifts of $N$ gives an exact functor

$$
\widehat{\mathcal{T}} \longrightarrow \operatorname{Mod}-E, \quad F \longmapsto F(N)^{*} .
$$

In more detail, for $i \in \mathbb{Z}$ we set $F(N)^{i}=F(N[-i])$ and we note that $E$ acts on $F(N)^{*}$ through $F$ since $F$ is an additive functor. The representable coherent functors (alias the projective objects in $\widehat{\mathcal{T}}$ ) evaluate to the realizable $E$-modules: for an object $M \in \mathcal{T}$ we have $\mathcal{T}(-, M)(N)^{*}=\mathcal{T}(N, M)^{*}$.

The relationship between Freyd's map (3.12) and the obstruction $\kappa$ can now be illustrated by a commutative diagram: for two coherent functors $F$ and $G$, the following diagram commutes, essentially by the definitions of $\nu$ and $\kappa$ :

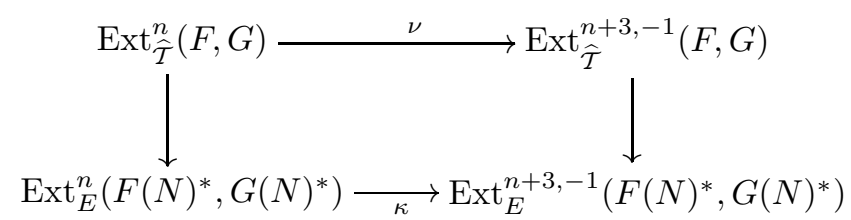

The vertical maps are induced by evaluation at $N$. Even though in general not every $E$-module is isomorphic to an $E$-module of the form $\mathcal{T}(N, M)^{*}$, they all arise from coherent functors. More is true: the evaluation functor has a left adjoint

$$
f: \operatorname{Mod}-E \longrightarrow \widehat{\mathcal{T}}
$$


which admits a natural isomorphism of graded $E$-modules

$$
\eta_{X}:(f X)(N)^{*} \stackrel{\cong}{\longrightarrow} X .
$$

The coherent functor $f X$ can be calculated from any $\mathcal{T}$-presentation

$$
\left(B[-1] \rightarrow R_{1} \stackrel{\alpha}{\rightarrow} R_{0} \rightarrow B, \varepsilon: \mathcal{T}\left(N, R_{0}\right)^{*} \rightarrow X\right)
$$

of $X$ as the cokernel of $\alpha_{*}: \mathcal{T}\left(-, R_{1}\right) \rightarrow \mathcal{T}\left(-, R_{0}\right)$. It is straightforward to check that $X \mapsto f X$ is actually functorial and independent of the choice of the $\mathcal{T}$-presentation. The map $\varepsilon: \mathcal{T}\left(N, R_{0}\right)^{*} \rightarrow X$ induces an isomorphism of graded $E$-modules $(f X)(N)^{*} \cong X$, by the defining property of a $\mathcal{T}$-presentation. Combining the commutativity of the diagram (3.13) with the natural isomorphism $\eta_{X}:(f X)(N)^{*} \rightarrow X$ gives the following alternative description of $\kappa$.

Corollary 3.14. The realizability obstruction

$$
\kappa: \operatorname{Hom}_{E}(X, Y) \longrightarrow \operatorname{Ext}_{E}^{3,-1}(X, Y)
$$

which is defined in Proposition $3.4(\mathrm{v})$ coincides with the composite

$$
\begin{aligned}
\operatorname{Hom}_{E}(X, Y) \stackrel{f}{\rightarrow} \operatorname{Hom}_{\widehat{\mathcal{T}}}(f X, f X) \stackrel{\nu}{\rightarrow} \operatorname{Ext}_{\widehat{\mathcal{T}}}^{3,-1}(f X, f Y) \\
\stackrel{\text { eval }}{\longrightarrow} \operatorname{Ext}_{E}^{3,-1}\left((f X)(N)^{*},(f Y)(N)^{*}\right) \stackrel{\eta}{\rightarrow} \operatorname{Ext}_{E}^{3,-1}(X, Y) .
\end{aligned}
$$

\section{Review of Hochschild COHOMOlogy}

Let $\Lambda$ be a $\mathbb{Z}$-graded algebra over the field $k$. Suppose further that $M$ is a graded $\Lambda$ - $\Lambda$-bimodule, with scalars from $k$ acting symmetrically. Then Hochschild cohomology of $\Lambda$ with coefficients in $M$ is the cohomology of a certain graded cochain complex $C^{\bullet}, *(\Lambda, M)$ defined by

$$
C^{n, m}(\Lambda, M)=\operatorname{Hom}_{k}^{m}\left(\Lambda^{\otimes n}, M\right),
$$

the space of graded $k$-linear maps from $\Lambda^{\otimes n}$ to $M$ of degree $m$ (as before, tensor products are taken over $k$ ). We index the first grading by a ' $\bullet$ ' and the second grading by ' $*$ ' since the differential only changes the first of these. So a Hochschild $(n, m)$-cochain with coefficients in $M$ is given by a multilinear function from $n$ tuples of elements of $\Lambda$ to $M$ which raise the degree by $m$. There is a differential $\delta: C^{n, m}(\Lambda, M) \rightarrow C^{n+1, m}(\Lambda, M)$ of bidegree $(1,0)$ given by

$$
\begin{aligned}
& (\delta \varphi)\left(\lambda_{1}, \ldots, \lambda_{n+1}\right)=(-1)^{m\left|\lambda_{1}\right|} \lambda_{1} \varphi\left(\lambda_{2}, \ldots, \lambda_{n+1}\right) \\
& \quad+\sum_{i=1}^{n}(-1)^{i} \varphi\left(\lambda_{1}, \ldots, \lambda_{i} \lambda_{i+1}, \ldots, \lambda_{n+1}\right)+(-1)^{n+1} \varphi\left(\lambda_{1}, \ldots, \lambda_{n}\right) \lambda_{n+1} .
\end{aligned}
$$

Finally, the Hochschild cohomology groups $H H^{*, *}(\Lambda, M)$ are the cohomology groups of the complex $C^{\bullet}, *(\Lambda, M)$,

$$
H H^{s, t}(\Lambda, M)=H^{s}\left(C^{\bullet, t}(\Lambda, M)\right) .
$$

One writes $H H^{s, t}(\Lambda)$ for $H H^{s, t}(\Lambda, \Lambda)$. For example, a class in $H H^{3,-1}(\Lambda)$ is represented by a family

$$
m=\left\{m_{i, j, l}: \Lambda^{i} \otimes \Lambda^{j} \otimes \Lambda^{l} \rightarrow \Lambda^{i+j+l-1}\right\}_{i, j, l \in \mathbb{Z}}
$$


of $k$-linear maps which satisfies the cocycle condition

$$
\begin{aligned}
(-1)^{|w|} w \cdot m(x, y, z)-m(w x, y, z) & +m(w, x y, z) \\
& -m(w, x, y z)+m(w, x, y) \cdot z=0
\end{aligned}
$$

for all homogenous elements $w, x, y, z$ of $\Lambda$. The complex $C^{\bullet \cdot *}(\Lambda, M)$ and its cohomology groups $H H^{*, *}(\Lambda, M)$ are covariant functors in the $\Lambda$-bimodule $M$. But they are also contravariant functors in the graded algebra: if $\alpha: \Gamma \rightarrow \Lambda$ is a homomorphism of graded $k$-algebras, we can view a $\Lambda$-bimodule $M$ as a $\Gamma$-bimodule through $\alpha$. Then precomposition with tensor powers of $\alpha$ gives a cochain homomorphism $\alpha^{*}: C^{\bullet}, *(\Lambda, M) \rightarrow C^{\bullet}, *(\Gamma, M)$, hence induced maps in Hochschild cohomology

$$
\alpha^{*}: H H^{s, t}(\Lambda, M) \rightarrow H H^{s, t}(\Gamma, M) \text {. }
$$

The second, internal grading in Hochschild cohomology can be traded in for a shift in the coefficient module, but this shifting involves a possibly unexpected sign. If $\Lambda$ and $\Gamma$ are $\mathbb{Z}$-graded $k$-algebras and $M$ is a graded $\Lambda$ - $\Gamma$-bimodule, then $M[1]$ is given by $M[1]^{n}=M^{n+1}$ as a graded $k$-vector space; we use the notation $\Sigma m$ when we view $m \in M^{n+1}$ as an element in $M[1]^{n}$. We make $M[1]$ into a $\Lambda$ - $\Gamma$-bimodule by setting

$$
\lambda \cdot(\Sigma m) \cdot \gamma=(-1)^{|\lambda|} \Sigma(\lambda m \gamma) .
$$

Note that the left action of $\Lambda$ is twisted. With this bimodule structure there is a natural isomorphism of chain complexes

$$
C^{\bullet, m}(\Lambda, M) \cong C^{\bullet, 0}(\Lambda, M[m])
$$

and hence a natural isomorphism of Hochschild cohomology groups

$$
H H^{s, t}(\Lambda, M) \cong H H^{s, 0}(\Lambda, M[t]) .
$$

The sign which occurs in (4.3) arises from the identification (i.e., isomorphism of categories) between right $\Lambda$-modules and left modules over the opposite algebra $\Lambda^{o p}$, where the multiplication in $\Lambda^{o p}$ is defined by

$$
\lambda \cdot \bar{\lambda}=(-1)^{|\lambda||\bar{\lambda}|} \bar{\lambda} \lambda .
$$

A graded right $\Lambda$-module $M$ can be viewed as a left $\Lambda^{o p}$-module by setting

$$
m \cdot \lambda=(-1)^{|\lambda||m|} \lambda m .
$$

If one defines a shift functor $M \longmapsto M[1]$ for right modules by degree-shifting with action $(\Sigma m) \cdot \lambda=\Sigma(m \lambda)$ (no extra sign), then the sign in (4.3) appears when translating right $\Lambda$-modules into left modules over $\Lambda^{o p}$. The sign can also be deduced from the Koszul sign convention by assigning the degree 1 to the suspension symbol $\Sigma$.

Next we define the cup product pairing

$$
\cup: \operatorname{Hom}_{\Lambda}(X, Y) \otimes H H^{*, *} \Lambda \longrightarrow \operatorname{Ext}_{\Lambda}^{* * *}(X, Y) \text {. }
$$

For the purpose of this paper, elements of Ext groups are represented by exact sequences, so we describe the cup product pairing from this point of view. To make the connection, we have to recall the bar complex $\mathcal{B}(\Lambda)$., a specific projective $\Lambda^{e}$ resolution of $\Lambda$; here we write $\Lambda^{e}$ for $\Lambda^{\mathrm{op}} \otimes \Lambda$, so that right $\Lambda^{e}$-modules are the same as $\Lambda$ - $\Lambda$-bimodules. The bar complex is given by $\mathcal{B}(\Lambda)_{n}=\Lambda^{\otimes(n+2)}$, with $\Lambda^{e}$-module structure given by

$$
\left(\lambda_{0}, \ldots, \lambda_{n+1}\right)\left(\mu, \mu^{\prime}\right)=(-1)^{|\mu|\left|\lambda_{0} \cdots \lambda_{n+1}\right|}\left(\mu \lambda_{0}, \lambda_{1}, \ldots, \lambda_{n}, \lambda_{n+1} \mu^{\prime}\right),
$$


and with differential defined by

$$
d\left(\lambda_{0}, \ldots, \lambda_{n+1}\right)=\sum_{i=0}^{n}(-1)^{i}\left(\lambda_{0}, \ldots, \lambda_{i} \lambda_{i+1}, \ldots, \lambda_{n+1}\right) .
$$

For the fact that $\mathcal{B}(\Lambda)$. is indeed a projective $\Lambda^{e}$-resolution of $\Lambda$, and for other details, see Cartan and Eilenberg [14], §IX.6. The Hochschild complex $C^{\bullet, 0}(\Lambda, M)$ is isomorphic to the complex $\operatorname{Hom}_{\Lambda^{e}}(\mathcal{B}(\Lambda) \bullet, M)$; it follows that Hochschild cohomology is isomorphic to bimodule Ext-groups,

$$
H H^{s, t}(\Lambda, M) \cong \operatorname{Ext}_{\Lambda^{e}}^{s}(\Lambda, M[t])
$$

Now suppose that $\varphi \in C^{n, m} \Lambda$ is a Hochschild cocycle and $f: X \rightarrow Y$ is a homomorphism of graded right $\Lambda$-modules. Under the identification

$$
C^{\bullet, m}(\Lambda) \cong \operatorname{Hom}_{\Lambda^{e}}(\mathcal{B}(\Lambda) \bullet, \Lambda[m]),
$$

the cocycle $\varphi$ corresponds to a bimodule homomorphism $\varphi: \mathcal{B}(\Lambda)_{n} \rightarrow \Lambda[m]$ satisfying $\varphi \circ d=0$. We tensor over $\Lambda$ with the homomorphism $f: X \rightarrow Y$ and obtain

$$
f \otimes_{\Lambda} \varphi: X \otimes \Lambda^{\otimes(n+1)} \cong X \otimes_{\Lambda} \mathcal{B}(\Lambda)_{n} \longrightarrow Y \otimes_{\Lambda} \Lambda[m] \cong Y[m] .
$$

This map is part of a diagram of right $\Lambda$-modules

$$
\begin{aligned}
& 0 \longrightarrow\left(X \otimes \Lambda^{\otimes(n+1)}\right) / \operatorname{Im}\left(X \otimes \Lambda^{\otimes(n+2)}\right) \longrightarrow \cdots \longrightarrow X \otimes \Lambda \longrightarrow X \longrightarrow 0 \\
& f \otimes_{\Lambda} \varphi \\
& Y[m]
\end{aligned}
$$

in which the upper row is exact. The base change of the upper exact sequence is an extension of $X$ by $Y[m]$ of length $n$. The class of this extension in $\operatorname{Ext}_{\Lambda}^{n, m}(X, Y)$ only depends on the cohomology class of the cocycle $\varphi$, and it defines $f \cup[\varphi]$.

Remark 4.5. There are other ways to describe the cup product pairing (4.4). The Hochschild cohomology groups are isomorphic to bimodule Ext-groups, which in turn can be described as morphisms in the derived category $\mathcal{D}\left(\Lambda^{e}\right)$ of $\Lambda$-bimodules,

$$
H H^{*}(\Lambda, M) \cong \operatorname{Ext}_{\Lambda^{e}}^{*}(\Lambda, M) \cong[\Lambda, M]_{\mathcal{D}\left(\Lambda^{e}\right)}^{*}
$$

Similarly, Ext groups between two left $\Lambda$-modules can be described as morphisms in the derived category $\mathcal{D}(\Lambda)$ of right $\Lambda$-modules. The derived tensor product for complexes of $\Lambda$-modules provides a bi-exact functor

$$
-\otimes_{\Lambda}^{L}-: \mathcal{D}(\Lambda) \times \mathcal{D}\left(\Lambda^{e}\right) \longrightarrow \mathcal{D}(\Lambda) .
$$

A special case of this is the map

$$
-\otimes_{\Lambda}^{L}-:[X, Y]_{\mathcal{D}(\Lambda)}^{*} \times[\Lambda, \Lambda]_{\mathcal{D}\left(\Lambda^{e}\right)}^{*} \longrightarrow[X, Y]_{\mathcal{D}(\Lambda)}^{*},
$$

which is another form of the cup product pairing. 


\section{The CANONICAL ClASS OF A DIFFERENTIAL GRADED ALGEBRA}

Any differential graded algebra $A$ over a field $k$ gives rise to a canonical class

$$
\gamma_{A} \in H H^{3,-1}\left(H^{*} A\right)
$$

in the Hochschild cohomology of the cohomology algebra $H^{*} A$. The class in the Hochschild cohomology of the Tate-cohomology $\operatorname{ring} \hat{H}^{*}(G, k)$ is a special case of this, since the Tate-cohomology has a natural underlying differential graded algebra; see Section 6.4. For the convenience of the reader, we recall the construction of $\gamma_{A}$ and we collect some of its properties in this section.

Construction 5.1. Since we are working over a field $k$, we can choose a quasiisomorphism of chain complexes $f_{1}: H^{*} A \longrightarrow A$ which induces the identity in cohomology (where we regard $H^{*} A$ as a complex of $k$-vector spaces with zero differential). This amounts to choosing a representative cocycle for each cohomology class, in a linear fashion.

Now $f_{1}$ usually cannot be chosen to commute with multiplication, but at least it commutes up to coboundaries. So we can choose a $k$-linear map of degree -1

$$
f_{2}: H^{*} A \otimes H^{*} A \longrightarrow A
$$

satisfying

$$
d f_{2}(x, y)=f_{1}(x) f_{1}(y)-f_{1}(x y) .
$$

Then for all homogenous $x, y, z \in H^{*} A$, the expression

$$
f_{2}(x, y) f_{1}(z)-f_{2}(x, y z)+f_{2}(x y, z)-(-1)^{|x|} f_{1}(x) f_{2}(y, z)
$$

is a cocycle in $A$. We let $m:\left(H^{*} A\right)^{\otimes 3} \longrightarrow H^{*} A$ denote the map of degree -1 which sends $x \otimes y \otimes z$ to the cohomology class of the expression (5.3).

The above Construction 5.1 depends on some choices, but our next task is to prove that it determines a Hochschild cohomology class which does not depend on these choices.

Proposition 5.4. $\quad$ (i) Let $A$ be a differential graded $k$-algebra and let

$$
f_{1}^{A}: H^{*} A \longrightarrow A \text { and } f_{2}^{A}:\left(H^{*} A\right)^{\otimes 2} \longrightarrow A[-1]
$$

be $k$-linear maps which satisfy (5.2). Then $m^{A}$ as defined by formula (5.3) is a Hochschild $(3,-1)$-cocycle of the graded algebra $H^{*} A$.

(ii) Let $B$ be another a differential graded $k$-algebra, let $\left(f_{1}^{B}, f_{2}^{B}\right)$ be maps for $B$ satisfying (5.2) and let $m^{B}:\left(H^{*} B\right)^{\otimes 3} \longrightarrow H^{*} B[-1]$ be defined by (5.3) from $\left(f_{1}^{B}, f_{2}^{B}\right)$. Suppose that $\alpha: H^{*} A \rightarrow H^{*} B$ is a homomorphism of graded $k$-algebras which is induced by some homomorphism of underlying differential graded $k$-algebras. Then the Hochschild $(3,-1)$-cocycles

$$
\alpha \circ m^{A} \quad \text { and } \quad m^{B} \circ \alpha^{\otimes 3}
$$

are cohomologous in the complex $C^{\bullet,-1}\left(H^{*} A, H^{*} B\right)$ (where $H^{*} B$ is a bimodule over $H^{*}$ A through $\alpha$ ).

Proof. (i) The cocycle condition, i.e., (4.2) for $m^{A}$, is a straightforward calculation using (5.2) and (5.3).

(ii) Suppose that $\rho: A \rightarrow B$ is a homomorphism of differential graded $k$-algebras which induces $\alpha=\rho_{*}: H^{*} A \rightarrow H^{*} B$ on cohomology. The maps $f_{1}^{B} \circ \rho_{*}$ and $\rho \circ f_{1}^{A}$ 
are chain maps from $H^{*} A$ (with trivial differential) to $B$, which induce the same map on cohomology; hence there is a $k$-linear map

$$
s: H^{*} A \longrightarrow B
$$

of degree -1 which satisfies

$$
d s=f_{1}^{B} \circ \rho_{*}-\rho \circ f_{1}^{A} .
$$

Another direct calculation shows that the expression

$$
\begin{aligned}
& \rho\left(f_{2}^{A}(x, y)\right)-f_{2}^{B}\left(\rho_{*}(x), \rho_{*}(y)\right)-s(x y) \\
&+(-1)^{|x|} \rho\left(f_{1}^{A}(x)\right) s(y)+s(x) f_{1}^{B}\left(\rho_{*}(y)\right)
\end{aligned}
$$

is a cocycle in $B$ for all $x, y \in H^{*} A$. So we can define $t \in C^{2,-1}\left(H^{*} A, H^{*} B\right)$ by sending $x \otimes y \in\left(H^{*} A\right)^{\otimes 2}$ to the cohomology class of the expression (5.6). A final, somewhat lengthy calculation then yields

$$
\rho_{*} \circ m^{A}+\partial t=m^{B} \circ\left(\rho_{*}\right)^{\otimes 3}
$$

in the Hochschild complex of $H^{*} A$ with coefficients in $H^{*} B[-1]$.

Corollary 5.7. $\quad$ (i) The Hochschild cohomology class represented by $m$ as in (5.3) is independent of the choices of the maps $f_{1}$ and $f_{2}$. We denote this Hochschild cohomology class by

$$
\gamma_{A} \in H H^{3,-1}\left(H^{*} A\right) .
$$

(ii) Let $A$ and $B$ be differential graded $k$-algebras, and let $\alpha: H^{*} A \rightarrow H^{*} B$ be induced by some homomorphism of underlying differential graded algebras. Then the canonical classes are related by the formula

$$
\alpha_{*}\left(\gamma_{A}\right)=\alpha^{*}\left(\gamma_{B}\right) \text { in } H H^{3,-1}\left(H^{*} A, H^{*} B\right)
$$

(where $H^{*} B$ is a bimodule over $H^{*} A$ through $\alpha$ ).

(iii) If $\rho: A \longrightarrow B$ is a quasi-isomorphism of differential graded algebras, then the class $\gamma_{A}$ is mapped to the class $\gamma_{B}$ under the induced isomorphism between the Hochschild cohomology of $H^{*} A$ and $H^{*} B$.

(iv) Suppose that the differential graded algebra $A$ is formal, i.e., quasi-isomorphic to its cohomology algebra with trivial differential. Then the class $\gamma_{A}$ is trivial.

Proof. Part (i) follows from Proposition 5.4(ii) by taking $A=B$ and letting $\alpha$ be the identity. Part (ii) is the content of Proposition 5.4 (ii). Part (iii) is a special case of (ii). Part (iv) is a special case of (iii) since the canonical class of a differential graded algebra with trivial differential vanishes.

Remark 5.8. According to Corollary 5.7, every differential graded algebra gives rise to a canonical Hochschild cohomology class. But not every class in the $(3,-1)$ Hochschild cohomology of a graded algebra $\Lambda$ actually arises in this fashion; the theory of $A_{\infty}$-algebras exhibits a sequence of obstructions. The first in the sequence of obstructions can be made explicit as follows. First one may check that for any Hochschild $(3,-1)$-cocycle $m$, the cochain

$$
m(m \otimes 1 \otimes 1+1 \otimes m \otimes 1+1 \otimes 1 \otimes m) \in C^{5,-2} \Lambda
$$


is again a cocycle, and that the cohomology class of (5.9) in $H H^{5,-2} \Lambda$ only depends on the class of $m$; the resulting quadratic map

$$
\text { Sq }: H H^{3,-1} \Lambda \longrightarrow H H^{5,-2} \Lambda
$$

is one of the operations introduced by Gerstenhaber in [22]. We claim that if a class $\gamma \in H H^{3,-1} \Lambda$ arises as the canonical class of a differential graded algebra, then the class $\mathrm{Sq}(\gamma) \in H H^{5,-2} \Lambda$ is trivial.

To prove the claim we use Kadeishvili's theorem [25] about the $A_{\infty}$-structure on the cohomology of a differential graded algebra; see also [29, Sec. 3.2]. By definition, an $A_{\infty}$-structure on a graded algebra $\Lambda$ consists of Hochschild cochains $m_{n} \in C^{n, 2-n} \Lambda$ for $n \geq 3$, such that the following relations hold:

$$
\sum_{\substack{i+j+l=n \\ i, j \leq 0, l \geq 1}}(-1)^{i+j l} m_{i+1+l} \circ\left(1^{\otimes i} \otimes m_{j} \otimes 1^{\otimes l}\right)=0 ;
$$

here the understanding is that $m_{1}=0$ and $m_{2}$ is the product of $\Lambda$. Given a differential graded algebra $A$, Kadeishvili's theorem provides such an $A_{\infty}$-structure on the cohomology $H^{*} A$ in which $m_{3}$ is a Hochschild $(3,-1)$-cocycle representing the canonical class $\gamma_{A}$.

Because $m_{1}=0$, the defining relation for $n=3$ just says that the product in $\Lambda$ is associative. The relation for $n=4$ specializes to the Hochschild cocycle condition for $m_{3}$. The defining relation for $n=5$ specializes to

$$
\begin{aligned}
& m_{3}\left(m_{3} \otimes 1 \otimes 1+1 \otimes m_{3} \otimes 1+1 \otimes 1 \otimes m_{3}\right)=m_{2}\left(1 \otimes m_{4}-m_{4} \otimes 1\right) \\
& -m_{4}\left(m_{2} \otimes 1 \otimes 1 \otimes 1+1 \otimes m_{2} \otimes 1 \otimes 1-1 \otimes 1 \otimes m_{2} \otimes 1+1 \otimes 1 \otimes 1 \otimes m_{2}\right) .
\end{aligned}
$$

The left-hand side of this equation represents $\mathrm{Sq}\left(\gamma_{A}\right)$; the right-hand side is precisely the Hochschild coboundary $\partial\left(m_{4}\right) \in C^{5,-2} \Lambda$, so the class $\operatorname{Sq}\left(\gamma_{A}\right)$ is trivial, as claimed.

Remark 5.10. The Hochschild cohomology class $\gamma_{A}$ contains information about all triple Massey products in the cohomology of the differential graded algebra $A$. We recall how these triple Massey products are defined. Let $M$ be a differential graded right $A$-module, for example $A$ as a module over itself. Let $x \in H^{*} M$ and $y, z \in H^{*} A$ be homogenous cohomology classes which satisfy

$$
x y=0=y z .
$$

Then the triple Massey product $\langle x, y, z\rangle$ is a coset of the group

$$
x \cdot\left(H^{|y|+|z|-1} A\right)+\left(H^{|x|+|y|-1} M\right) \cdot z
$$

in the group $H^{|x|+|y|+|z|-1} M$.

To define the Massey product $\langle x, y, z\rangle$, choose representing cocycles $a \in M$ and $b, c \in A$ for the classes $x, y$ and $z$, respectively. Because of the relations (5.11), there exist cochains $u \in M^{|x|+|y|-1}$ and $v \in A^{|y|+|z|-1}$ satisfying $d(u)=a b$ and $d(v)=b c$. For any such choices, the cochain

$$
u c-(-1)^{|x|} a v
$$

is a cocycle, and $\langle x, y, z\rangle$ is defined as the set of cohomology classes represented by the expressions (5.12) for all choices of $a, b, c, u$ and $v$.

We need one important relation between triple Massey products. Suppose that in addition to $x, y$ and $z$ as above we have another cohomology class $w \in H^{*} A$ 
which satisfies $z w=0$. Then the brackets $\langle x, y, z\rangle$ and $\langle y, z, w\rangle$ are defined with appropriate indeterminacies, and the juggling formula

$$
x \cdot\langle y, z, w\rangle=(-1)^{|x|+1}\langle x, y, z\rangle \cdot w
$$

holds as cosets of $x \cdot\left(H^{*} A\right) \cdot w$. We omit the straightforward proof.

Lemma 5.14. Let $A$ be a differential graded algebra and let $m \in C^{3,-1}\left(H^{*} A\right)$ be a Hochschild cocycle which represents the canonical class $\gamma_{A}$. Then for all homogenous elements $x, y, z \in H^{*} A$ such that $x y=0=y z$, the class $m(x, y, z)$ is contained in the triple Massey product $\langle x, y, z\rangle$.

Proof. If we change the Hochschild cocycle $m$ by a coboundary, then the value of $m(x, y, z)$ is changed by an element in $x \cdot H^{*} A+H^{*} A \cdot z$, the indeterminacy group of the Massey product. Indeed if $t \in C^{2,-1}\left(H^{*} A\right)$ is a Hochschild 2-cochain, then

$$
(\delta t)(x, y, z)=(-1)^{|x|} x \cdot t(y, z)-t(x, y) \cdot z
$$

because of the relations (5.11). Hence it suffices to prove the claim for a single choice of cocycle which represents the class $\gamma_{A}$. We do this verification for a cocycle $m$ constructed in Construction 5.1

If we choose $f_{1}$ and $f_{2}$ as in Construction 5.1 then $f_{1}(x), f_{1}(y)$ and $f_{1}(z)$ are representing cocycles for the classes $x, y$ and $z$, and we have

$$
d f_{2}(x, y)=f_{1}(x) f_{1}(y) \text { and } d f_{2}(y, z)=f_{1}(y) f_{1}(z)
$$

because $x y=0=y z$. So the Massey product $\langle x, y, z\rangle$ contains the class

$$
\left[f_{2}(x, y) f_{1}(z)-(-1)^{|x|} f_{1}(x) f_{2}(y, z)\right]=m(x, y, z) \text {. }
$$

Example 5.15. The obstruction classes $\kappa(X) \in \operatorname{Ext}_{H^{*} A}^{3,-1}(X, X)$ are defined from the triangulated structure of the derived category $\mathcal{D}(A)$ of differential graded $A$ modules. We shall see in the next section (Theorem 6.2) that the classes $\kappa(X)$ all arise from the canonical class $\gamma_{A} \in H H^{3,-1}\left(H^{*} A\right)$ via the relation $\kappa(X)=\operatorname{id}_{X} \cup \gamma_{A}$. However, in general there is no hope to recover the Hochschild cohomology class $\gamma_{A}$ from the triangulated category $\mathcal{D}(A)$. To illustrate this we now describe a differential graded algebra $A$ such that

(i) the class $\gamma_{A}$ is non-trivial, but

(ii) the derived category of $\mathcal{D}(A)$ is equivalent, as a triangulated category, to the derived category of the cohomology algebra $H^{*} A$, considered as a dga with trivial differential.

In the example, the cohomology of $A$ is a Laurent polynomial algebra on a generator of degree 1, over an inseparable field extension of $k$. Thus every graded module over $H^{*} A$ is free, and all Ext-groups over $H^{*} A$ in positive dimensions are trivial.

We let $k=\mathbb{F}_{2}(x)$ be the function field in one variable $x$ over the field with two elements. We first define the degree 0 algebra by setting

$$
A^{0}=k\langle u, a\rangle /\left(a^{2}, a u+u a+1\right),
$$

the free associative $k$-algebra on two non-commuting variables $u$ and $a$ subject to the two specified relations. Equivalently, $A^{0}$ could be defined as the algebra of $2 \times 2$-matrices over the polynomial algebra $k[z]$, where the generators correspond to

$$
u=\left(\begin{array}{ll}
0 & z \\
1 & 0
\end{array}\right) \quad \text { and } \quad a=\left(\begin{array}{ll}
0 & 1 \\
0 & 0
\end{array}\right) .
$$


As a graded $k$-algebra, $A$ is the Laurent polynomial algebra over $A^{0}$ in one indeterminate $v$ of degree 1 ,

$$
A=A^{0} \otimes k\left[v^{ \pm 1}\right] .
$$

Finally, the differential on $A$ is determined by

$$
d(u)=d(v)=0 \quad \text { and } \quad d(a)=\left(u^{2}-x\right) \cdot v
$$

(recall that $x \in \mathbb{F}_{2}(x)=k$ is the function field variable). Because $d(a)$ is in the center of $A$, we have

$$
d(a) \cdot a+a \cdot d(a)=0 \quad \text { and } \quad d(a) \cdot u+u \cdot d(a)+d(1)=0,
$$

and so $d$ does indeed extend to a differential satisfying the Leibniz formula on all of $A$.

We let $\bar{u} \in H^{0} A$ denote the cohomology class of the cocycle $u \in A^{0}$, and, by abuse of notation, $v \in H^{1} A$ also denotes the cohomology class of the cocycle $v \in A^{1}$.

Proposition 5.16. The cohomology algebra of $A$ is a Laurent polynomial algebra in one indeterminate of degree 1 over the field obtained from $k$ by adjoining a square root of $x$,

$$
H^{*} A=k[\bar{u}] /\left(\bar{u}^{2}-x\right)\left[v^{ \pm 1}\right] .
$$

The canonical $(3,-1)$-Hochschild cohomology class $\gamma_{A}$ is non-trivial.

Proof. The Laurent generator $v \in A^{1}$ is a cocycle and it is central in $A$, so its class in $H^{1} A$ is a central unit of the cohomology algebra. Hence it suffices to show that $H^{0} A$, the cohomology algebra in degree 0 , is isomorphic to the quadratic extension field $k[\bar{u}] /\left(\bar{u}^{2}-x\right)$.

We note that $u$ generates a polynomial sub-algebra of $A^{0}$ and as a left module (and also as a right module) over $k[u]$, the algebra $A^{0}$ is free of rank two with basis $\{1, a\}$. More generally, $A^{n}$ is free of rank two over $k[u]$ with basis $\left\{v^{n}, a v^{n}\right\}$ for every $n \in \mathbb{Z}$. Since $u$ is a cocycle, the differential of $A$ is left $k[u]$-linear. Since $d(1)=0$ and $d(a)=\left(u^{2}-x\right) v$, the kernel of $d: A^{0} \rightarrow A^{1}$ is precisely the polynomial subalgebra $k[u]$. Similarly, the image of $d: A^{-1} \rightarrow A^{0}$ is generated as a $k[u]$-module by $d\left(a v^{-1}\right)=u^{2}-x$. Hence we have

$$
H^{0} A=k[\bar{u}] /\left(\bar{u}^{2}-x\right),
$$

as claimed.

In order to describe a representing cocycle for the canonical Hochschild class $\gamma_{A}$, we specify $f_{1}, f_{2}$ and $m$ on the $k$-basis $\left\{v^{n}, \bar{u} v^{n}\right\}_{n \in \mathbb{Z}}$ of $H^{*} A$ and extend $k$-linearly. The obvious choice for $f_{1}$ is

$$
f_{1}\left(v^{n}\right)=v^{n} \quad \text { and } \quad f_{1}\left(\bar{u} v^{n}\right)=u v^{n} .
$$

This choice of $f_{1}$ is multiplicative except for pairs of basis elements of the form $\left(\bar{u} v^{n}, \bar{u} v^{m}\right)$, where we have

$$
f_{1}\left(\bar{u} v^{n}\right) f_{1}\left(\bar{u} v^{m}\right)-f_{1}\left(\bar{u} v^{n} \cdot \bar{u} v^{m}\right)=\left(u^{2}-x\right) \cdot v^{n+m} .
$$

So we define $f_{2}$ by

$$
f_{2}\left(u v^{n}, u v^{m}\right)=a v^{n+m-1}
$$

and setting $f_{2} \equiv 0$ on all other pairs of basis elements. Then the expression

$$
f_{2}\left(\lambda_{1}, \lambda_{2}\right) f_{1}\left(\lambda_{3}\right)-f_{2}\left(\lambda_{1}, \lambda_{2} \lambda_{3}\right)+f_{2}\left(\lambda_{1} \lambda_{2}, \lambda_{3}\right)-(-1)^{\left|\lambda_{1}\right|} f_{1}\left(\lambda_{1}\right) f_{2}\left(\lambda_{2}, \lambda_{3}\right)
$$


vanishes for all choices of basis elements $\lambda_{i}$, except when all three elements are of the form $\bar{u} v^{n}$. In that latter case we get

$$
\begin{aligned}
m\left(\bar{u} v^{n}, \bar{u} v^{m}, \bar{u} v^{l}\right)= & {\left[f_{2}\left(\bar{u} v^{n}, \bar{u} v^{m}\right) f_{1}\left(\bar{u} v^{l}\right)-f_{2}\left(\bar{u} v^{n}, \bar{u}^{2} v^{m+l}\right)\right.} \\
& \left.+f_{2}\left(\bar{u}^{2} v^{n+m}, \bar{u} v^{l}\right)-(-1)^{n} f_{1}\left(\bar{u} v^{n}\right) f_{2}\left(\bar{u} v^{m}, \bar{u} v^{l}\right)\right] \\
= & {\left[(a u+u a) v^{n+m+l-1}\right]=v^{n+m+l-1} }
\end{aligned}
$$

(using for example that $f_{2}\left(\bar{u}, \bar{u}^{2}\right)=x \cdot f_{2}(\bar{u}, 1)=0$ ).

It remains to show that this Hochschild 3-cocycle $m$ is not a coboundary. By inspection, the coboundary of every $k$-linear map $t \in C^{2,-1}\left(H^{*} A\right)$ satisfies

$$
(\delta t)(\bar{u}, \bar{u}, \bar{u})=x \cdot(\delta t)((\bar{u}, 1,1)+(1,1, \bar{u})) .
$$

Since $m(\bar{u}, \bar{u}, \bar{u})$ is different from $x \cdot m((\bar{u}, 1,1)+(1,1, \bar{u}))$, the cocycle $m$ cannot be a coboundary. We conclude that the canonical class $\gamma_{A}$ is indeed non-trivial.

The point of this example depends on the following lemma.

Lemma 5.17. Let $A$ be a differential graded algebra such that $H^{0} A$ is a field and $H^{1} A$ contains a unit. Then the zeroth cohomology functor

$$
H^{0}: \mathcal{D}(A) \longrightarrow \operatorname{Mod}-\left(H^{0} A\right)
$$

is an equivalence from the derived category of differential graded right A-modules to the category of vector spaces over $H^{0} A$.

Proof. Let $M$ be a differential graded $A$-module. Since $H^{0}$ is a field, we may choose a set $\left\{m_{i}\right\}_{i \in I}$ of zero-cocycles in $M$ whose cohomology classes form a basis of $H^{0} M$ over $H^{0} A$. These cocycles determine a unique homomorphism

$$
\bigoplus_{I} A \longrightarrow M
$$

from the free differential graded $A$-module to $M$ which sends the $i$ th basis element to $m_{i}$. This morphism induces an isomorphism in cohomology in dimension zero. Since $H^{1} A$ contains a unit, the morphism (5.18) is thus a quasi-isomorphism of differential graded $A$-modules. In other words, every differential graded $A$-module is quasi-isomorphic to a free module.

Now we show that for every pair of differential graded $A$-modules $M$ and $N$,

$$
H^{0}:[M, N]_{\mathcal{D}(A)} \rightarrow \operatorname{Hom}_{H^{0} A}\left(H^{0} M, H^{0} N\right)
$$

is bijective. This is clear when $M=A$ is the free module of rank one, in which case both sides identify with $H^{0} N$. For fixed $N$, the two functors $[-, N]_{\mathcal{D}(A)}$ and $\operatorname{Hom}_{H^{0} A}\left(H^{0}(-), H^{0} N\right)$ take arbitrary sums in $\mathcal{D}(A)$ to products of $k$-vector spaces. So the map (5.19) is bijective whenever $M$ is a free module. By the first part, every differential graded $A$-module is isomorphic in $\mathcal{D}(A)$ to a free module, so the map (5.19) is always bijective.

This shows that the functor $H^{0}$ is fully faithful, and it remains to show that the image is dense. Since $H^{0}$ is a field, every $H^{0}$-module has a basis, and is thus isomorphic to the zeroth cohomology of the free differential graded $A$-module on a basis of the same cardinality. So the functor $H^{0}$ is an equivalence of categories. 
Lemma 5.17 is a special case of a more general phenomenon: if $A$ is a differential graded algebra such that every graded module over the graded cohomology algebra $H^{*} A$ is projective, then the functor

$$
H^{*}: \mathcal{D}(A) \longrightarrow \operatorname{Mod}-\left(H^{*} A\right)
$$

is an equivalence of categories to the category of graded modules over $H^{*} A$. If $H^{1} A$ contains a unit, then graded modules over $H^{*} A$ are equivalent to (ordinary) modules over $H^{0} A$.

Now we sum up what this example is showing. The cohomology algebra of the $\operatorname{dga} A$ is a Laurent polynomial algebra over a field on a generator of degree 1 . So by Lemma 5.17 the cohomology functor

$$
H^{0}: \mathcal{D}(A) \longrightarrow \operatorname{Mod}-\left(H^{0} A\right)
$$

is a $k$-linear equivalence from the derived category of differential graded right $A$ modules to the category of vector spaces over the field $H^{0} A=k[\sqrt{x}]$. Lemma 5.17 also applies to the derived category of $H^{*} A$, with trivial differential; we conclude that the derived categories of $A$ and $H^{*} A$ are equivalent as triangulated categories. Nevertheless, the canonical $(3,-1)$-Hochschild cohomology class of $A$ is non-trivial whereas the class of $H^{*} A$ is trivial (since it has trivial differential).

Example 5.20. Suppose that we are given a graded $k$-algebra $\Lambda$ and two integers $s$ and $t$. Then for every Hochschild cohomology class $\gamma \in H H^{s, t} \Lambda$, the collection of classes $\operatorname{id}_{X} \cup \gamma \in \operatorname{Ext}_{\Lambda}^{s, t}(X, X)$ for all graded $\Lambda$-modules $X$ are compatible in the sense that

$$
\beta \circ\left(\operatorname{id}_{X} \cup \gamma\right)=\left(\operatorname{id}_{Y} \cup \gamma\right) \circ \beta \in \operatorname{Ext}_{\Lambda}^{n+s, t}(X, Y)
$$

for every class $\beta \in \operatorname{Ext}_{\Lambda}^{n}(X, Y)$, where the circle denotes Yoneda composition. The previous Example 5.15] shows in particular that this map from $H H^{s, t} \Lambda$ to collections of compatible classes in $\operatorname{Ext}_{\Lambda}^{s, t}(X, X)$ need not be injective. We demonstrate by another example that in general, this map is also not surjective. Here the algebras are actually ungraded, so the second (internal) grading is omitted.

Let $H$ be the 9-dimensional $k$-algebra which is the subalgebra of $M_{5}(k)$ consisting of all matrices $\left(a_{i j}\right)$ with non-zero entries only if $i=j$ or $i=1$. This is a tame hereditary algebra, and its category of finite-dimensional modules is wellunderstood; see Section 3.6 in [36. We fix an indecomposable module $X_{0}$ having an Auslander-Reiten sequence $\kappa_{0} \in \operatorname{Ext}_{H}^{1}\left(X_{0}, X_{0}\right)$. For every direct sum $X=\bigoplus_{I} X_{0}$ of copies of $X_{0}$, we define

$$
\kappa(X)=\bigoplus_{I} \kappa_{0} \in \operatorname{Ext}_{H}^{1}(X, X) .
$$

An arbitrary $H$-module $X$ has, up to isomorphism, a unique decomposition $X=$ $X^{\prime} \oplus X^{\prime \prime}$, where $X^{\prime}$ is isomorphic to a direct sum of copies of $X_{0}$ and $X^{\prime \prime}$ has no direct summand isomorphic to $X_{0}$. We view $\kappa\left(X^{\prime}\right)$ as an element in $\operatorname{Ext}_{H}^{1}(X, X)$ and define $\kappa(X)=\kappa\left(X^{\prime}\right)$. We have

$$
f \circ \kappa(X)=\kappa(Y) \circ f \in \operatorname{Ext}_{H}^{1}(X, Y)
$$

for every homomorphism $f: X \longrightarrow Y$ since $\kappa_{0}$ is an Auslander-Reiten sequence. More precisely, $\kappa(X)$ is an element in the socle of $\operatorname{Ext}_{H}^{1}(X, X)$, where $\operatorname{Ext}_{H}^{1}(X, X)$ is viewed as an $\operatorname{End}_{H}(X)$-module (acting either from the left or from the right). It follows that $f \circ \kappa(X)=0$ except when $f$ is invertible. Thus the computation of 
$f \circ \kappa(X)$ reduces to scalar multiplication in the socle of $\operatorname{Ext}_{H}^{1}\left(X_{0}, X_{0}\right)$, which is 1dimensional. The field $k$ is acting centrally and this shows that $f \circ \kappa(X)=\kappa(Y) \circ f$. Now observe that the algebra $H$ is hereditary. Therefore all Ext groups in dimension 2 and above are trivial. So $\kappa$ is also natural for Yoneda composition with Ext classes. However there exists no $\gamma \in H H^{1}(H)$ such that $\kappa(X)=\operatorname{id}_{X} \cup \gamma$ for all $H$-modules $X$ since $H H^{1}(H)=0$.

\section{Comparing $\gamma_{A}$ With $\kappa(f)$ in DERIVED AND Stable MOdules CATEGORIES}

In this section we state our main theorems; they say that in the case of the derived category of a differential graded algebra, and for the stable module category of a selfinjective algebra, the obstruction classes $\kappa(f)$ constructed in Theorem [3.7 all arise from a single "global" cohomology class, namely the canonical $(3,-1)$-Hochschild cohomology class defined in Corollary 5.7.

6.1. Derived categories. During this section we fix a differential graded $k$ algebra $A$. The free module of rank one is a compact object in the derived category $\mathcal{D}(A)$ of differential graded $A$-modules, so the theory of Section 3 applies. Moreover, the graded endomorphism algebra $\mathcal{D}(A)(A, A)^{*}$ is canonically isomorphic to the cohomology algebra $H^{*} A$, via the evaluation map

$$
\mathcal{D}(A)(A, A)^{*} \rightarrow H^{*} A, \quad f \longmapsto H^{*}(f)(1) .
$$

We use the evaluation to identify the two algebras. Moreover, the analogous evaluation map $\mathcal{D}(A)(A, X)^{*} \rightarrow H^{*} X$ is an isomorphism of $H^{*} A$-modules if we view the source as an $H^{*} A$-module via the evaluation isomorphism.

Theorem 6.2. Let $A$ be a differential graded $k$-algebra and let

$$
\gamma_{A} \in H H^{3,-1}\left(H^{*} A\right)
$$

be the canonical Hochschild cohomology class. Then for two graded $H^{*} A$-modules $X$ and $Y$ the realizability obstruction

$$
\kappa: \operatorname{Hom}_{H^{*} A}(X, Y) \longrightarrow \operatorname{Ext}_{H^{*} A}^{3,-1}(X, Y)
$$

of Theorem 3.7 is given by the cup product

$$
\kappa(f)=f \cup \gamma_{A} .
$$

This theorem will be proved in Section 9. In the course of the proof we also construct a functor

$$
\Psi: \operatorname{Mod}-\left(H^{*} A\right) \longrightarrow \mathcal{D}(A)
$$

from the category of graded $H^{*} A$-modules to the derived category of differential graded $A$-modules together with a natural monomorphism of graded $H^{*} A$ modules $\eta_{X}: X \longrightarrow H^{*}(\Psi X)$. The module $\Psi X$ features in a functorial special $\mathcal{D}(A)$-presentation of $X$ with further nice properties; see Remark [9.7. It follows from part (iii) of Proposition 3.4 that for any map of graded $H^{*} A$-modules $f: X \longrightarrow H^{*} M$ there exists a map $\beta: \Psi X \longrightarrow M$ in the derived category $\mathcal{D}(A)$ such that $f=H^{*}(\beta) \circ \eta_{X}$.

Altogether this implies

Corollary 6.3. Let $A$ be a differential graded $k$-algebra and $f: X \longrightarrow Y$ a morphism of graded modules over the graded cohomology algebra $H^{*} A$. Then the following are equivalent: 
(i) The morphism $f$ factors over a realizable $H^{*} A$-module.

(ii) There exists a map of graded $H^{*} A$-modules $g: H^{*}(\Psi X) \longrightarrow Y$ such that $f=g \circ \eta_{X}$.

(iii) The class $f \cup \gamma_{A}$ in $\operatorname{Ext}_{H^{*} A}^{3,-1}(X, Y)$ is trivial.

In particular, a graded $H^{*} A$-module $X$ is a direct summand of a realizable module if and only if the class $\operatorname{id}_{X} \cup \gamma_{A} \in \operatorname{Ext}_{H^{*} A}^{3,-1}(X, X)$ is trivial.

Proof. Theorem 6.2 shows that the realizability obstruction $\kappa(f)$ of Theorem 3.7 agrees with the class $f \cup \gamma_{A}$. Hence conditions (i) and (iii) are equivalent by Theorem 3.7. Condition (i) is a special case of condition (ii). Conversely, condition (ii) implies condition (i) by Proposition 3.4(iii).

6.4. Stable module categories. Now we consider a Frobenius algebra $L$ over the field $k$ (i.e., an algebra such that the classes of projective and injective left $L$-modules coincide), and we work in the stable module category StMod- $L$ of right $L$-modules. Moreover we fix an $L$-module $N$ which is finitely generated - this ensures that $N$ is compact as an object of the stable module category. We are interested in realizing graded modules over the Tate Ext-algebra $E=\widehat{\operatorname{Ext}}_{L}^{*}(N, N)$. For example, $L$ can be the group algebra $k G$ of a finite group $G$ and $N$ can be the trivial $k G$-module, in which case $E=\widehat{\operatorname{Ext}}_{k G}^{*}(k, k)=\hat{H}^{*}(G, k)$ is the Tate cohomology algebra of $G$.

In this situation we can consider a certain differential graded algebra, namely the endomorphism dga of a complete resolution $\hat{P}_{*}$ of $N$. In other words, $\hat{P}_{*}$ is obtained by splicing together a projective resolution and an injective resolution of $N$

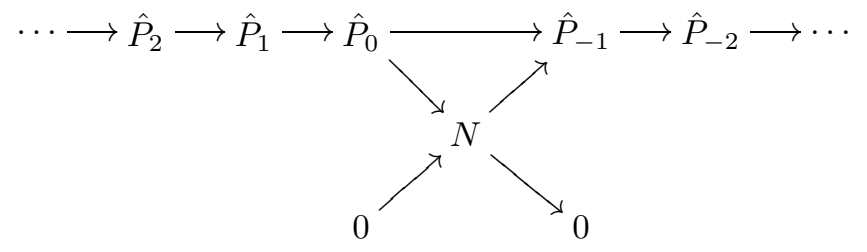

Denote by $A$ the cochain complex

$$
A=\operatorname{Hom}_{L}^{\bullet}\left(\hat{P}_{*}, \hat{P}_{*}\right)
$$

so that

$$
A^{n}=\prod_{j} \operatorname{Hom}_{L}\left(\hat{P}_{n+j}, \hat{P}_{j}\right)
$$

with differential $d: A^{n} \longrightarrow A^{n+1}$ defined by

$$
(d f)(x)=\partial(f(x))-(-1)^{n} f(\partial(x)),
$$

where $\partial$ denotes the differential of $\hat{P}_{*}$. With this definition, the $n$-cocycles in $A$ are the chain homomorphisms of degree $n$ (anti-chain homomorphisms for odd $n$ ), and cocycles differ by a coboundary if and only if the corresponding (anti-)chain maps are homotopic. So $A$ is a differential graded algebra whose cohomology $H^{*} A$ is the Tate Ext algebra $\widehat{\operatorname{Ext}}_{L}^{*}(N, N)$. But more is true: by a Theorem of Keller [28] 4.3], there is a triangle equivalence between the localizing subcategory of StMod- $L$ generated by $N$ and the derived category of differential graded $A$-modules. We will not use Keller's result, but we borrow his method in the proof of Theorem 6.9 below. 
Lemma 6.6. The quasi-isomorphism type of the endomorphism differential graded algebra $\operatorname{Hom}_{L}^{\bullet}\left(\hat{P}_{*}, \hat{P}_{*}\right)$ does not depend on the choice of the complete resolution $\hat{P}_{*}$ of $N$.

Proof. Suppose $\hat{Q}_{*}$ is another complete resolution of $N$ by projective $L$-modules. Then $\hat{P}_{*}$ and $\hat{Q}_{*}$ are chain homotopy equivalent as complexes of $L$-modules. By replacing $\hat{P}_{*}$ by the mapping cylinder of some homotopy equivalence $\hat{P}_{*} \rightarrow \hat{Q}_{*}$ we can assume that there exists a surjective chain homotopy equivalence $f: \hat{P}_{*} \rightarrow \hat{Q}_{*}$. We let $E(f)$ be the endomorphism differential graded algebra of the homotopy equivalence $f$, defined as the pullback in the category of complexes of $L$-modules

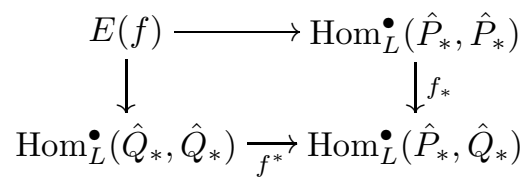

In other words, an $n$-cochain of $E(f)$ consists of two maps of graded $L$-modules $\alpha_{1}$ : $P[n] \rightarrow P$ and $\alpha_{2}: Q[n] \rightarrow Q$ such that $\alpha_{1} f=f[n] \alpha_{2}$. Then $E(f)$ is a differential graded algebra under composition and the two maps to the endomorphism dgas of $\hat{P}_{*}$ and $\hat{Q}_{*}$ are dga-homomorphisms. Since $f$ is a chain homotopy equivalence, so are the two maps $f_{*}$ and $f^{*}$ in the defining pullback (6.7) for $E(f)$. Since $f$ is surjective and $\hat{P}_{*}$ consists of projective modules, the right vertical map $f_{*}$ is also surjective. So its base change $E(f) \rightarrow \operatorname{Hom}_{L}^{\bullet}\left(\hat{Q}_{*}, \hat{Q}_{*}\right)$ is a quasi-isomorphism, and so is the fourth map in the square (6.7).

Definition 6.8. Let $L$ be a Frobenius $k$-algebra and $N$ a finitely generated $L$ module. We denote by

$$
\gamma_{N / L} \in H H^{3,-1}\left(\widehat{\operatorname{Ext}}_{L}^{*}(N, N)\right)
$$

the canonical Hochschild cohomology class (see Corollary 5.7) of the endomorphism differential graded algebra of a complete resolution of $N$ over $L$. By Lemma 6.6 and Corollary 5.7(iii), this class is independent of the choice of complete resolution.

Theorem 6.9. Let $L$ be a self-injective $k$-algebra, $N$ a finite-dimensional $L$-module and let

$$
E=\widehat{\operatorname{Ext}}_{L}^{*}(N, N)
$$

denote the graded Tate Ext algebra of $N$. Then for two graded E-modules $X$ and $Y$ the realizability obstruction

$$
\kappa: \operatorname{Hom}_{E}(X, Y) \longrightarrow \operatorname{Ext}_{E}^{3,-1}(X, Y)
$$

of Theorem 3.7 defined in the stable module category of $L$ is given by the cup product

$$
\kappa(f)=f \cup \gamma_{N / L} .
$$

In particular, a graded $E$-module $X$ is a direct summand of a realizable module if and only if the class $\operatorname{id}_{X} \cup \gamma_{N / L} \in \operatorname{Ext}_{E}^{3,-1}(X, X)$ is trivial.

Proof. We reduce the problem to the case of derived categories and appeal to Theorem 6.2 The method is the same as in Keller's paper [28, 4.3], where stable module categories are interpreted as derived categories over suitable endomorphism dgas. As in the definition of the class $\gamma_{N / L}$, we choose a complete resolution $\hat{P}_{*}$ of $N$ and 
let $A=\operatorname{Hom}_{L}^{\bullet}\left(\hat{P}_{*}, \hat{P}_{*}\right)$ denote the endomorphism dga of the resolution. Then we have a functor

$$
\operatorname{Hom}_{L}^{\bullet}\left(\hat{P}_{*},-\right): \operatorname{Mod}-L \longrightarrow \operatorname{Mod}-A
$$

from right $L$-modules to differential graded right $A$-modules. Since $\hat{P}_{*}$ consists of projective modules, the functor $\operatorname{Hom}_{L}^{\bullet}\left(\hat{P}_{*},-\right)$ is exact and it comes equipped with a natural isomorphism of graded $E$-modules

$$
H^{*}\left(\operatorname{Hom}_{L}^{\bullet}\left(\hat{P}_{*}, B\right)\right) \cong \widehat{\operatorname{Ext}}_{L}^{*}(N, B)
$$

for $B \in \operatorname{Mod}-L$. Thus the functor $\operatorname{Hom}_{L}^{\bullet}\left(\hat{P}_{*},-\right)$ takes projective $L$-modules to acyclic $A$-modules, and it passes to a well-defined derived functor

$$
\operatorname{RHom}_{L}\left(\hat{P}_{*},-\right): \text { StMod- } L \longrightarrow \mathcal{D}(A)
$$

on the level of triangulated categories. We abbreviated this derived functor to

$$
F: \text { StMod- } L \longrightarrow \mathcal{D}(A) \text {. }
$$

Moreover, there is a chain of quasi-isomorphisms of differential graded $A$-modules between

$$
\operatorname{Hom}_{L}^{\bullet}\left(\hat{P}_{*}, \Omega B\right) \text { and } \operatorname{Hom}_{L}^{\bullet}\left(\hat{P}_{*}, B\right)[-1]
$$

which yields a natural isomorphism

$$
F(\Omega B) \cong F(B)[-1]
$$

in the derived category of $A$. With respect to this isomorphism, $F$ preserves distinguished triangles, so it becomes an exact functor of triangulated categories.

Now let $X$ be a graded module over the Tate Ext algebra $E$. Choose a StMod- $L$ presentation

$$
\left(\Omega B \stackrel{\delta}{\rightarrow} R_{1} \stackrel{\alpha}{\rightarrow} R_{0} \stackrel{\pi}{\rightarrow} B, \varepsilon: \widehat{\operatorname{Ext}}_{L}^{*}\left(N, R_{0}\right) \longrightarrow X\right)
$$

(cf. Definition 3.1). Applying the exact functor $F$ yields a $\mathcal{D}(A)$-presentation

$$
\begin{aligned}
\left(F(B)[-1] \cong F(\Omega B) \stackrel{F(\delta)}{\longrightarrow} F\left(R_{1}\right) \stackrel{F(\alpha)}{\longrightarrow} F\left(R_{0}\right) \stackrel{F(\pi)}{\longrightarrow} F(B),\right. \\
\left.\bar{\varepsilon}: H^{*} F\left(R_{0}\right) \cong \widehat{\operatorname{Ext}}_{L}^{*}\left(N, R_{0}\right) \stackrel{\varepsilon}{\rightarrow} X\right) .
\end{aligned}
$$

The natural isomorphism (6.10) shows that the Yoneda class of the extension

$$
0 \longrightarrow X[-1] \longrightarrow \widehat{\operatorname{Ext}}_{L}^{*}(N, \Omega B) \longrightarrow \widehat{\operatorname{Ext}}_{L}^{*}\left(N, R_{1}\right) \longrightarrow \widehat{\operatorname{Ext}}_{L}^{*}\left(N, R_{0}\right) \stackrel{\varepsilon}{\rightarrow} X \longrightarrow 0
$$

is equal to the Yoneda class of

$$
0 \longrightarrow X[-1] \longrightarrow H^{*} F(\Omega B) \longrightarrow H^{*} F\left(R_{1}\right) \longrightarrow H^{*} F\left(R_{0}\right) \stackrel{\bar{\varepsilon}}{\rightarrow} X \longrightarrow 0 .
$$

So we conclude that the obstruction class associated to $X$ via the stable module category of $L$ is the same as the class coming from the derived category of the endomorphism dga $A$; so using Theorem 6.2 we conclude that

$$
\kappa_{\mathrm{StMod}-L}(X)=\kappa_{\mathcal{D}(A)}(X)=\operatorname{id}_{X} \cup \gamma_{N / L} .
$$

For a homomorphism $f: X \rightarrow Y$ of graded $E$-modules, we get the formal consequence

$$
\kappa(f)=f \circ \kappa\left(\operatorname{id}_{X}\right)=f \circ\left(\operatorname{id}_{X} \cup \gamma_{N / L}\right)=f \cup \gamma_{N / L} .
$$


Remarks 6.11. (i) One can also apply the theory to the problem of realizing modules over ordinary cohomology, as opposed to Tate cohomology, but the result is not quite what one might want. Suppose that $L$ is a $k$-algebra (not necessarily Frobenius), and let $N$ be an $L$-module which has a finite resolution by finitely generated projective $L$-modules; this assures that $N$ is compact as an object of the unbounded derived category $\mathcal{D}(L)$ of $L$. Let $E=\operatorname{Ext}_{L}^{*}(N, N)$ denote the Ext algebra of $N$. Then for every $L$-module $M$, the Ext groups $\operatorname{Ext}_{L}^{*}(N, M)$ are a graded $E$-module by Yoneda composition. But there are more realizable modules in the sense of Section 3 for every complex $C_{*}$ of $L$-modules, the hyper Ext-groups $\mathbb{E x t}_{L}^{*}\left(N, C_{*}\right)=\mathcal{D}(L)\left(N, C_{*}\right)^{*}$ are realizable, graded $E$-modules.

Given a graded $E$-module $X$, Theorem 3.7 provides a necessary and sufficient homological condition for when $X$ is a summand of an $E$-module of the form $\mathbb{E x t}_{L}^{*}\left(N, C_{*}\right)$. The obstruction $\kappa(X)$ also arises from a Hochschild cohomology class, namely the canonical class of the endomorphism dga of a projective resolution of $N$ over $L$; the proof is very similar to the proof of Theorem 6.9 But one might want to know when $X$ is a summand of the Ext groups of an individual module, not just of a complex of modules. Our approach does not give new insight into this problem.

(ii) There are other models for the differential graded algebra underlying the Tate Ext algebra. Suppose as before that $\hat{P}_{*}$ is a complete resolution of an $L$-module $N$. We say that an $n$-cochain $f \in \operatorname{Hom}_{L}^{n}\left(\hat{P}_{*}, \hat{P}_{*}\right)$, i.e., a graded map of degree $n$, is eventually trivial if its components $f_{j}: \hat{P}_{n+j} \rightarrow \hat{P}_{j}$ are trivial for $j \gg 0$. Now the eventually trivial cochains form a differential graded ideal $T$ in $\operatorname{Hom}_{L}^{\bullet}\left(\hat{P}_{*}, \hat{P}_{*}\right)$. Moreover, every chain map which is eventually trivial is necessarily null-homotopic; this just says that the ideal $T$ is acyclic, so the projection map

$$
\operatorname{Hom}_{L}^{\bullet}\left(\hat{P}_{*}, \hat{P}_{*}\right) \longrightarrow \operatorname{Hom}_{L}^{\bullet}\left(\hat{P}_{*}, \hat{P}_{*}\right) / T
$$

is a quasi-isomorphism of dgas.

This smaller model is variously attributed to Vogel [23, Mislin [32], Benson and Carlson [9]. It also receives a homomorphism from the endomorphism dga of an ordinary resolution $P_{*}$ (as opposed to a complete resolution) of $N$. Indeed, any graded map $f: P[n] \rightarrow P$ can be extended trivially to a graded map between complete resolutions. This gives a map of graded $k$-algebras

$$
\operatorname{Hom}_{L}^{\bullet}\left(P_{*}, P_{*}\right) \longrightarrow \operatorname{Hom}_{L}^{\bullet}\left(\hat{P}_{*}, \hat{P}_{*}\right)
$$

which is not compatible with the differential. However, the deviation from commuting with the differential is eventually trivial, so the composite map with the projection

$$
\operatorname{Hom}_{L}^{\bullet}\left(P_{*}, P_{*}\right) \longrightarrow \operatorname{Hom}_{L}^{\bullet}\left(\hat{P}_{*}, \hat{P}_{*}\right) / T
$$

is a homomorphism of dgas. On cohomology, this latter map realizes the $k$-algebra homomorphism from ordinary Ext groups to Tate Ext groups.

(iii) Complete resolutions can also be used for Kropholler's LH $\mathcal{F}$-groups. Complete cohomology can be defined for arbitrary rings by stabilizing Ext with respect to dimension shifting. This is explained in the context of group rings of arbitrary groups in Benson and Carlson [9, Mislin [32]. But often it is not clear whether it can be calculated using complete resolutions. Kropholler [30] shows that in the context of modules of type $F P_{\infty}$ over group rings of groups in a very large class 
denoted $\mathbf{L H} \mathcal{F}$, complete cohomology can be calculated using complete resolutions. See also Cornick and Kropholler [16], [17, Benson [7], 8].

(iv) A theorem of Rickard [35] shows that any localization of $\hat{H}^{*}(G, k)$ is realizable by an idempotent $k G$-module. More generally, any localization of a realizable module is again realizable. Benson and Gnacadja [10] have given an example of a flat $\hat{H}^{*}(G, k)$-module which is not realizable. This is related to the existence of filtered systems in StMod- $k G$ which do not lift to filtered systems in Mod- $k G$. For this purpose, it is essential that the field $k$ be uncountable.

\section{EXAMPLES}

We show in some simple cases how to identify the Hochschild class

$$
\gamma_{G} \in H H^{3,-1} \hat{H}^{*}(G, k)
$$

and hence how to decide when a module over the Tate cohomology of $G$ is a summand of a realizable module. Here we write $\gamma_{G}$ for $\gamma_{k / k G}$, defined as in Definition 6.8 from a complete resolution of the trivial $k G$-module. In characteristic $p$ we get complete control over all groups $G$ whose $p$-Sylow subgroup is cyclic.

We denote by $C_{n}$ the cyclic group of order $n$. The calculations of the Tate cohomology and the Hochschild classes for cyclic groups are all well known, but we reproduce them for the convenience of the reader. We will first identify the class $\gamma_{C_{p} m} \in H H^{3,-1} \hat{H}^{*}\left(C_{p^{m}}, k\right)$ for $p$ a prime, $k$ a field of characteristic $p$ and $m \geq 1$. The answer comes out differently according to whether $p^{m}=2, p^{m}=3$ or $p^{m} \geq 4$.

Theorem 7.1. Let $k$ be a field of positive characteristic $p$ and $m \geq 1$. If $p=2$ and $m=1$, then the Tate cohomology is a ring of Laurent polynomials on a 1dimensional class $x$,

$$
\hat{H}^{*}\left(C_{2}, k\right)=k\left[x^{ \pm 1}\right] .
$$

If $p^{m} \geq 3$, then the Tate cohomology $\hat{H}^{*}\left(C_{p^{m}}, k\right)$ is a tensor product of an exterior algebra on a 1-dimensional class $x$ and a Laurent polynomial algebra on a 2-dimensional class $y$,

$$
\hat{H}^{*}\left(C_{p^{m}}, k\right)=\Lambda(x) \otimes k\left[y^{ \pm 1}\right] .
$$

The canonical class

$$
\gamma_{C_{p^{m}}} \in H H^{3,-1} \hat{H}\left(C_{p^{m}}, k\right)
$$

is trivial except when $p=3$ and $m=1$. In the latter case, the class $\gamma_{C_{3}}$ is represented by the $(3,-1)$-cocycle $m$ of $\Lambda(x) \otimes k\left[y^{ \pm 1}\right]$ which satisfies

$$
m\left(x y^{i} \otimes x y^{j} \otimes x y^{l}\right)=y^{i+j+l+1}
$$

and which vanishes on all other tensor products of monomials in $x$ and $y$.

The calculation for cyclic groups is really a special case of the calculation for truncated polynomial algebras. In any characteristic, positive or zero, and for any $n \geq 1$, the truncated polynomial algebra $k[z] / z^{n}$ is self-injective, so our theory applies, and Definition 6.8 gives the canonical Hochschild cohomology class

$$
\gamma_{k /\left(k[z] / z^{n}\right)} \in H H^{3,-1}\left(\widehat{\operatorname{Ext}}_{k[z] / z^{n}}^{*}(k, k)\right) .
$$

If $n$ is a power of the characteristic of $k$, then the group algebra $k C_{n}$ and the truncated polynomial algebra $k[z] / z^{n}$ are isomorphic as augmented $k$-algebras. So Theorem 7.1 is a special case of the following theorem. 
Theorem 7.3. Let $L=k[z] / z^{n}$ be the truncated polynomial algebra of height $n$ over a field $k$ of any characteristic. If $n=2$, then the Tate cohomology algebra of $L$ is a ring of Laurent polynomials on a 1-dimensional class $x$,

$$
\widehat{\operatorname{Ext}}_{L}^{*}(k, k)=k\left[x^{ \pm 1}\right] .
$$

If $n \geq 3$, then the Tate cohomology algebra is a tensor product of an exterior algebra on a 1-dimensional class $x$ and a Laurent polynomial algebra on a 2-dimensional class $y$

$$
\widehat{\operatorname{Ext}}_{L}^{*}(k, k)=\Lambda(x) \otimes k\left[y^{ \pm 1}\right]
$$

The canonical class

$$
\gamma_{k / L} \in H H^{3,-1} \widehat{\operatorname{Ext}}_{L}^{*}(k, k)
$$

is trivial unless $n=3$. If $n=3$, the class $\gamma_{k / L}$ is non-trivial and is represented by the $(3,-1)$-cocycle $m$ of $\Lambda(x) \otimes k\left[y^{ \pm 1}\right]$ which satisfies

$$
m\left(x y^{i} \otimes x y^{j} \otimes x y^{l}\right)=y^{i+j+l+1}
$$

and which vanishes on all other tensor products of monomials in $x$ and $y$.

Before we prove Theorem 7.3, we illustrate it with a remark and some examples.

Remark 7.4. In the above calculation of the Tate-Ext algebra of a truncated polynomial algebra, the cases $n=2$ and $n=3$ seem to be exceptional, but that is just because we do not take into account the higher order information (for example in terms of an $A_{\infty}$-structure). The "exceptional" behavior is a special case of the relation

$$
m_{n}(\underbrace{x \otimes \cdots \otimes x}_{n})=y \quad \text { in } \quad \widehat{\operatorname{Ext}}_{k[z] / z^{n}}^{2}(k, k),
$$

where $m_{n}$ defines the $A_{n}$-structure on the Tate-Ext groups. The map $m_{2}$ is just the ordinary product, whence $y=x^{2}$ for $n=2$; any cocycle representing the canonical class $\gamma_{k / L}$ can be taken as the map $m_{3}$, so for $n=3$ this is the last statement in Theorem 7.3 .

Example 7.5 (Cyclic group of order 2). If $k$ is a field of characteristic 2, then the Tate cohomology $\hat{H}^{*}\left(C_{2}, k\right)$ is a ring of Laurent polynomials on a 1-dimensional class $x$,

$$
\hat{H}^{*}\left(C_{2}, k\right)=k\left[x^{ \pm 1}\right]
$$

and the canonical Hochschild class $\gamma_{C_{2}}$ is trivial by Theorem 7.1 The fact that every graded $\hat{H}^{*}\left(C_{2}, k\right)$-module is realizable, not only up to summands, can be seen directly: the Tate cohomology algebra $\hat{H}^{*}\left(C_{2}, k\right)$ is a graded field, i.e., every graded module over it is free. Hence the Tate cohomology functor

$$
\hat{H}^{0}\left(C_{2},-\right): \text { StMod- } k C_{2} \longrightarrow \operatorname{Mod}-\hat{H}^{0}\left(C_{2}, k\right)
$$

is an equivalence of categories, by Lemma 5.17 .

By Theorem 7.1, the canonical class $\gamma_{C_{p} m}$ is trivial if the characteristic of the field $k$ is not 3, or if $m \geq 2$. By the general theory, every graded module over the Tate cohomology algebra $\hat{H}^{*}\left(C_{p^{m}}, k\right)$ is then a direct summand of a realizable module. The same holds for modules over the truncated polynomial algebra $k[z] / z^{n}$ in any characteristic, as long as $n \neq 3$, by Theorem[7.3. We take a closer look at the remaining case, the truncated polynomial algebra of height 3 in any characteristic. 
Example 7.6. We let $k$ be any field, we set $L=k[z] / z^{3}$; if $k$ has characteristic 3, then $L$ is also isomorphic to the group algebra of the cyclic group of order 3 . We let $x \in \widehat{\operatorname{Ext}}_{L}^{1}(k, k)$ be the generator which satisfies

$$
x^{2}=0 \quad \text { and } \quad\langle x, x, x\rangle=y
$$

(by the relationship between the canonical class and three-fold Massey products; cf. Remark 5.10). If $M$ is any $L$-module and if a class $w \in \widehat{\operatorname{Ext}}_{L}^{i}(k, M)$ satisfies $w x=0$, then there is a Massey product

$$
\langle w, x, x\rangle \subseteq \widehat{\operatorname{Ext}}_{L}^{i+1}(k, M) ;
$$

cf. Remark 5.10. The juggling formula (5.13) gives

$$
w y=w \cdot\langle x, x, x\rangle=(-1)^{i+1}\langle w, x, x\rangle \cdot x \text { in } \widehat{\operatorname{Ext}}_{L}^{i+2}(k, M) .
$$

So $w=(-1)^{i+1}\langle w, x, x\rangle \cdot y^{-1} x$, and we conclude that every class in the Tate cohomology of a $k[z] / z^{3}$-module which is annihilated by $x$ is also divisible by $x$. This property is inherited by direct summands and it characterizes the free modules among all graded modules over $\Lambda(x) \otimes k\left[y^{ \pm 1}\right]$. So for a graded $\widehat{\operatorname{Ext}}_{L}^{*}(k, k)$-module $X$ the following three conditions are equivalent:

(i) $X$ is realizable.

(ii) $X$ is isomorphic to a direct summand of a realizable module.

(iii) $X$ is a free module.

In particular the cyclic $\widehat{\operatorname{Ext}}_{L}^{*}(k, k)$-module concentrated in even dimensions,

$$
X=\widehat{\operatorname{Ext}}_{L}^{*}(k, k) /(x),
$$

is not a direct summand of a realizable module.

Example 7.7 (Products of groups). There does not seem to be an easy way to calculate the canonical class for Tate-cohomology of a product of groups from the corresponding classes of the individual groups, in sharp contrast with the situation for ordinary group cohomology. If $G$ and $H$ are two finite groups and $k$ is a field, then the Kunneth theorem shows that the cohomology algebra $H^{*}(G \times H, k)$ is the tensor product of the cohomology algebras of $G$ and $H$. In fact, the Kunnethisomorphism has a precursor on the level of underlying differential graded algebras: if $P_{*}$ is a projective resolution of the trivial module over the group algebra $k G$, and if $Q_{*}$ is a similar projective resolution over the group algebra $k H$, then the tensor product $P_{*} \otimes Q_{*}$ is a projective resolution of the trivial module over $k[G \times H]$. Moreover, the differential graded endomorphism algebras of $P_{*}, Q_{*}$ and $P_{*} \otimes Q_{*}$ realize the cohomology algebras $H^{*}(G, k), H^{*}(H, k)$ and $H^{*}(G \times H, k)$, respectively. The Kunneth-isomorphism is then realized by the quasi-isomorphism of dgas

$$
\operatorname{Hom}_{k G}^{*}\left(P_{*}, P_{*}\right) \otimes \operatorname{Hom}_{k H}^{*}\left(Q_{*}, Q_{*}\right) \longrightarrow \operatorname{Hom}_{k[G \times H]}^{*}\left(P_{*} \otimes Q_{*}, P_{*} \otimes Q_{*}\right)
$$

given by tensoring endomorphisms.

Now there is a general product formula for the canonical class of the tensor product of two differential graded algebras $A$ and $B$. Suppose the canonical classes $\gamma_{A} \in H H^{3,-1}\left(H^{*} A\right)$ and $\gamma_{B} \in H H^{3,-1}\left(H^{*} B\right)$ are represented by $(3,-1)$-cocycles $m^{A}$ and $m^{B}$, respectively. Then one can show that under the Kunneth-isomorphism $H^{*}(A \otimes B) \cong H^{*} A \otimes H^{*} B$, the canonical class

$$
\gamma_{A \otimes B} \in H H^{3,-1}\left(H^{*} A \otimes H^{*} B\right)
$$


is represented by the cocycle

$$
\begin{aligned}
m\left(x_{1} \otimes y_{1}, x_{2} \otimes y_{2}, x_{3} \otimes y_{3}\right) & \\
= & \pm m^{A}\left(x_{1}, x_{2}, x_{3}\right) \otimes y_{1} y_{2} y_{3}+x_{1} x_{2} x_{3} \otimes m^{B}\left(y_{1}, y_{2}, y_{3}\right),
\end{aligned}
$$

where the correct sign can be determined from the Koszul sign rule.

The situation for Tate cohomology is quite different. We illustrate this by looking at the Klein four group $\mathbb{Z} / 2 \times \mathbb{Z} / 2$, over a field $k$ of characteristic 2 . By Theorem 7.1 the canonical class $\gamma_{\mathbb{Z} / 2}$ in the $(3,-1)$-Hochschild cohomology of the Tate cohomology of $\mathbb{Z} / 2$ is trivial; nevertheless, the canonical class for the product group $(\mathbb{Z} / 2)^{2}$ is non-trivial, which we show by exhibiting a non-trivial triple Massey product in $\hat{H}^{*}\left((\mathbb{Z} / 2)^{2}, k\right)$, and appealing to Remark 5.10

Again we find it convenient to move to a characteristic-free context by working over the self-injective algebra $L=k[z, w] /\left(z^{2}=w^{2}=0\right)$. If $k$ has characteristic 2 , then $L$ is isomorphic to the group algebra of $(\mathbb{Z} / 2)^{2}$. We claim that there exist non-trivial classes

$$
x, y \in \operatorname{Ext}_{L}^{1}(k, k) \text { and } \quad \phi \in \widehat{\operatorname{Ext}}_{L}^{-1}(k, k)
$$

satisfying $x \phi=0=\phi y$ and having Massey product

$$
\langle x, \phi, y\rangle=1
$$

in $\widehat{\operatorname{Ext}}_{L}^{0}(k, k)=\operatorname{Hom}(k, k)$ without indeterminacy. Hence the canonical class

$$
\gamma_{k / L} \in H H^{3,-1} \widehat{\operatorname{Ext}}_{L}^{*}(k, k)
$$

is non-trivial. It turns out that $\widehat{\operatorname{Ext}}_{L}^{*}(k, k)$ has no invertible elements except in dimension 0; so the map $\widehat{\operatorname{Ext}}_{L}^{*}(k, k) \rightarrow k$ which projects onto the degree zero part is a homomorphism of graded $k$-algebras, and $k$, concentrated in dimension 0 , becomes a graded module over $\widehat{\operatorname{Ext}}_{L}^{*}(k, k)$. Using the bracket (7.8), a similar argument as in Example 7.6 shows that $k$ is not a summand of any realizable module.

It remains to describe the classes $x, y$ and $\phi$, and to calculate the bracket $\langle x, \phi, y\rangle$. The syzygy module $\Omega k$ is the 3 -dimensional augmentation ideal

$$
\Omega k=(z, w)=\operatorname{kernel}[L \longrightarrow k] .
$$

We let $x \in \operatorname{Ext}_{L}^{1}(k, k)=\underline{\operatorname{Hom}}_{L}(\Omega k, k)$ be represented by the unique $L$-homomorphism $\bar{x}: \Omega k \rightarrow k$ satisfying $\bar{x}(z)=1$ and $\bar{x}(w)=0$. Similarly, $y$ is represented by $\bar{y}: \Omega k \rightarrow k$ satisfying $\bar{y}(w)=1$ and $\bar{y}(z)=0$. Then the (non-negative) Ext algebra $\operatorname{Ext}_{L}^{*}(k, k)$ is polynomial on the classes $x$ and $y$, but we will not use this. We let $\phi \in \widehat{\operatorname{Ext}}_{L}^{-1}(k, k)=\underline{\operatorname{Hom}}_{L}(k, \Omega k)$ be represented by the $L$-homomorphism $\bar{\phi}: k \rightarrow \Omega k$ determined by $\bar{\phi}(1)=z w \in \Omega k$. We have $\bar{x} \bar{\phi}=0=\bar{y} \bar{\phi}$; since the Tate Ext algebra is graded commutative, this yields $x \phi=0=\phi y$ and the Massey product (7.8) is defined. Since neither $x$ nor $y$ is a unit in the Tate Ext algebra, the bracket has no indeterminacy.

In order to calculate the bracket, we first have to factor the composite maps $\bar{\phi} \bar{y}: \Omega k \rightarrow \Omega k$ and $\bar{x} \bar{\phi}: k \rightarrow k$ through projective $L$-modules. The composite $\bar{\phi} \bar{y}$ factors as

$$
\Omega k \stackrel{\text { incl. }}{\longrightarrow} L \stackrel{H}{\longrightarrow} \Omega k,
$$

where $H$ is multiplication by $z \in \Omega k$. The other composite $\bar{x} \bar{\phi}$ is trivial, so we can factor through the trivial module, and so the bracket is represented by the unique 
factorization of $\bar{x} H: L \rightarrow k$ through the quotient $L / \Omega k \cong k$. Now $\bar{x} H$ is the augmentation, so the bracket $\langle x, \phi, y\rangle$ is indeed represented by the identity of $k$.

Proof of Theorem 7.3. We assume $n \geq 2$ throughout the proof and write $L=$ $k[z] / z^{n}$ for the truncated polynomial algebra of height $n$. A complete resolution $\hat{P}$ of the trivial $L$-module $k$, which is moreover 2-periodic and minimal, is given by $\hat{P}_{i}=L$, the free module of rank one, with differential $d_{i}: \hat{P}_{i} \rightarrow \hat{P}_{i-1}$ given by

$$
d_{i}= \begin{cases}\text { multiplication by }-z^{n-1} & \text { if } i \text { is even, and } \\ \text { multiplication by } z & \text { if } i \text { is odd. }\end{cases}
$$

The complex $\operatorname{Hom}_{L}(\hat{P}, k)$ has trivial differential, so the Tate cohomology $\widehat{\operatorname{Ext}}_{L}^{*}(k, k)$ is one-dimensional in every degree.

We let $\bar{x} \in \operatorname{Hom}_{L}^{1}(\hat{P}, \hat{P})$ be the anti-chain map $\hat{P}[1] \rightarrow \hat{P}$ which is the identity in even degrees and multiplication by $z^{n-2}$ in odd degrees; we let $x \in H^{1} \operatorname{Hom}_{L}^{*}(\hat{P}, \hat{P})$ $\cong \widehat{\operatorname{Ext}}_{L}^{1}(k, k)$ be the class of the anti-chain map $\bar{x}$. We let $\bar{y} \in \operatorname{Hom}_{L}^{2}(\hat{P}, \hat{P})$ be the periodicity isomorphism, i.e., the chain map $\hat{P}[2] \rightarrow \hat{P}$ which is the identity in every degree; we let $y \in H^{2} \operatorname{Hom}_{L}^{*}(\hat{P}, \hat{P}) \cong \widehat{\operatorname{Ext}}_{L}^{2}(k, k)$ be the class of the chain map $\bar{y}$. Since $y$ is invertible and the Tate cohomology is one-dimensional in every degree, the monomial $x^{\epsilon} y^{i}$ is a generator of $\widehat{\operatorname{Ext}}_{L}^{2 i+\epsilon}(k, k)$ for all $\epsilon \in\{0,1\}$ and $i \in \mathbb{Z}$.

In order to determine the multiplicative structure of $\widehat{\operatorname{Ext}}_{L}^{*}(k, k)$ it remains to calculate the square of the 1 -dimensional class $x$. The element $x^{2} \in \widehat{\operatorname{Ext}}_{L}^{2}(k, k)$ is represented by the chain map $\bar{x}^{2}: \hat{P}[2] \rightarrow \hat{P}$ which is multiplication by $z^{n-2}$ in every degree. If $n=2$, then we have $\bar{x}^{2}=\bar{y}$ on the level of chain maps, and thus $x^{2}=y$ in $\widehat{\operatorname{Ext}}_{L}^{2}(k, k)$. Thus $x^{i} \longmapsto \bar{x}^{i}$ defines a multiplicative cycle selection homomorphism, and the endomorphism dga is actually formal; in particular the canonical class is trivial.

From now on we assume $n \geq 3$; then $\bar{x}^{2}$ is null-homotopic (for example by the homotopy which is multiplication by $z^{n-3}$ in odd degrees and trivial in even degrees). So for $n \geq 3$ we get $x^{2}=0$, and the multiplicative structure $\widehat{\operatorname{Ext}}_{L}^{*}(k, k)$ is as claimed.

Now we determine a $(3,-1)$-cocycle which represents the canonical class in

$$
H H^{3,-1}\left(\Lambda(x) \otimes k\left[y^{ \pm 1}\right]\right) .
$$

We use the cycle selection homomorphism

$$
f_{1}: \Lambda(x) \otimes k\left[y^{ \pm 1}\right] \longrightarrow \operatorname{Hom}_{L}^{*}(\hat{P}, \hat{P})
$$

which is given on the monomial basis by

$$
f_{1}\left(x^{\epsilon} y^{i}\right)=\bar{x}^{\epsilon} \bar{y}^{i}: \hat{P}[2 j+\epsilon] \longrightarrow \hat{P} .
$$

Since the periodicity isomorphism $\bar{y}$ commutes with $\bar{x}$, this cycle selection homomorphism is multiplicative, except on two odd-dimensional classes. The product

$$
f_{1}\left(x y^{i}\right) f_{1}\left(x y^{j}\right)=\bar{x}^{2} \bar{y}^{i+j}: \hat{P}[2(i+j+1)] \longrightarrow \hat{P}
$$

is multiplication by $z^{n-2}$ in every degree, which is only null-homotopic. We define $f_{2}$ by

$$
f_{2}\left(y^{i}, y^{j}\right)=f_{2}\left(x y^{i}, y^{j}\right)=f_{2}\left(y^{i}, x y^{j}\right)=0
$$


for all $i, j \in \mathbb{Z}$. On tensor products of two odd degree elements we let

$$
f_{2}\left(x y^{i}, x y^{j}\right): \hat{P}[2(i+j)+1] \longrightarrow \hat{P}
$$

be multiplication by $z^{n-3}$ in odd degrees, and multiplication by 0 in even degrees. Then $f_{2}\left(x y^{i}, x y^{j}\right)$ is a chain null-homotopy for $f_{1}\left(x y^{i}\right) f_{1}\left(x y^{j}\right)$, so $f_{1}$ and $f_{2}$ satisfy the condition (5.2), and we can use these maps in the formula (5.3) and obtain a representing cocycle $m$ for $\gamma_{A}$. Then the cocycle $m$ defined by (5.3) vanishes on tensor products in which at least one monomial has even degree. On tensor products of three odd degree elements,

$$
m\left(x y^{i}, x y^{j}, x y^{l}\right) \in \widehat{\operatorname{Ext}}_{L}^{2(i+j+l+1)}(\hat{P}, \hat{P})
$$

is represented by the chain map

$$
f_{2}\left(x y^{i}, x y^{j}\right) f_{1}\left(x y^{l}\right)+f_{1}\left(x y^{i}\right) f_{2}\left(x y^{j}, x y^{l}\right): \hat{P}[2(i+j+l+1)] \longrightarrow \hat{P}
$$

which is multiplication by $z^{n-3}$ in all degrees. If $n \geq 4$, then that chain map is null-homotopic (for example by the homotopy which is multiplication by $z^{n-4}$ is even degrees and trivial in odd degrees). Therefore, for $n \geq 4$ the $(3,-1)$-cocycle representing $\gamma_{A}$ is trivial, hence so is the class $\gamma_{A}$ itself.

Finally if $n=3$, then $m\left(x y^{i}, x y^{j}, x y^{l}\right)=y^{i+j+l+1}$. Hence the class of $m$ in $H H^{3,-1}\left(\Lambda(x) \otimes k\left[y^{ \pm 1}\right]\right)$ is non-trivial since every coboundary of a $(2,-1)$-cochain vanishes on the tensor product of three odd degree elements.

\section{Reduction to Sylow subgroups}

In this section we show that if $G$ is a finite group and $k$ is a field of characteristic $p>0$, then the canonical class

$$
\gamma_{G} \in H H^{3,-1}\left(\hat{H}^{*}(G, k)\right)
$$

is determined by the corresponding class of the $p$-Sylow subgroup of $G$. This allows us to determine the canonical class $\gamma_{G}$ for all finite groups $G$ with cyclic $p$-Sylow subgroup.

Throughout this section, the field $k$ is fixed, so we drop it from the notation and write $\hat{H}^{*}(G)$ for $\hat{H}^{*}(G, k)$, and similarly for the Tate cohomology of the Sylow subgroup. If $G$ is a finite group and $P$ is any subgroup of $G$, then there is a restriction map

$$
\text { res : } \hat{H}^{*}(G) \longrightarrow \hat{H}^{*}(P)
$$

which is a homomorphism of graded $k$-algebras, as well as a transfer map

$$
\operatorname{tr}: \hat{H}^{*}(P) \longrightarrow \hat{H}^{*}(G) ;
$$

see for example [14], Chap. XII.8. The composite tr $\circ$ res is multiplication by the index $[G: P]$, so if this index is prime to the characteristic of $k$, then restriction is injective. Moreover we have the reciprocity formula

$$
\operatorname{tr}(x \cdot \operatorname{res}(y))=\operatorname{tr}(x) \cdot y
$$

for $x \in \hat{H}^{*}(P)$ and $y \in \hat{H}^{*}(G)$.

Because the restriction map is a homomorphism of graded algebras, it makes $\hat{H}^{*}(P)$ into a bimodule over $\hat{H}^{*}(G)$ and induces a restriction map

$$
\text { res }^{*}: H H^{s, t}\left(\hat{H}^{*}(P)\right) \longrightarrow H H^{s, t}\left(\hat{H}^{*}(G), \hat{H}^{*}(P)\right)
$$


in Hochschild cohomology. The reciprocity formula (8.1) says that the transfer map is a homomorphism of $\hat{H}^{*}(G)$-modules if we let $\hat{H}^{*}(G)$ act on $\hat{H}^{*}(P)$ through restriction. The Tate-cohomology algebras are graded commutative, so the transfer map is in fact a homomorphism of $\hat{H}^{*}(G)$-bimodules, and so it induces another map in Hochschild cohomology

$$
\operatorname{tr}_{*}: H H^{s, t}\left(\hat{H}^{*}(G), \hat{H}^{*}(P)\right) \longrightarrow H H^{s, t}\left(\hat{H}^{*}(P)\right) \text {. }
$$

Theorem 8.3. Let $k$ be a field of characteristic $p, G$ a finite group and $P$ a $p$-Sylow subgroup of $G$. Then the canonical class $\gamma_{G} \in H H^{3,-1}\left(\hat{H}^{*}(G)\right)$ is determined by the canonical class $\gamma_{P} \in H H^{3,-1}\left(\hat{H}^{*}(P)\right)$ of the Sylow subgroup by the formula

$$
\gamma_{G}=\frac{\operatorname{tr}_{*}\left(\operatorname{res}^{*}\left(\gamma_{P}\right)\right)}{[G: P]} .
$$

Proof. The key point is that the restriction map in Tate cohomology is in fact induced by a homomorphism of differential graded algebras. Indeed, if we let $\hat{Q}_{*}$ be a complete resolution of the trivial module by projective $k G$-modules, then by neglect of structure, $\hat{Q}_{*}$ is also a complete resolution of the trivial module by projective $k P$-modules. So we have an inclusion of differential graded endomorphism algebras

$$
\operatorname{Hom}_{k G}^{\bullet}\left(\hat{Q}_{*}, \hat{Q}_{*}\right) \longrightarrow \operatorname{Hom}_{k P}^{\bullet}\left(\hat{Q}_{*}, \hat{Q}_{*}\right)
$$

whose effect on cohomology

$$
\hat{H}^{*}(G) \cong H^{*}\left(\operatorname{Hom}_{k G}^{\bullet}\left(\hat{Q}_{*}, \hat{Q}_{*}\right)\right) \longrightarrow H^{*}\left(\operatorname{Hom}_{k P}^{\bullet}\left(\hat{Q}_{*}, \hat{Q}_{*}\right)\right) \cong \hat{H}^{*}(P)
$$

is the restriction map. By part (ii) of Corollary 5.7 we have the relation

$$
\operatorname{res}^{*}\left(\gamma_{P}\right)=\operatorname{res}_{*}\left(\gamma_{G}\right)
$$

in $H H^{3,-1}\left(\hat{H}^{*}(G), \hat{H}^{*}(P)\right)$. Applying the transfer in Hochschild cohomology (8.2) gives

$$
\operatorname{tr}_{*}\left(\operatorname{res}^{*}\left(\gamma_{P}\right)\right)=\operatorname{tr}_{*}\left(\operatorname{res}_{*}\left(\gamma_{G}\right)\right)=[G: P] \cdot \gamma_{G} .
$$

The result follows since the index of $P$ in $G$ is invertible in $k$.

Over a field of positive characteristic $p$, the canonical Hochschild cohomology class $\gamma_{C_{p} m}$ of the cyclic group of order $p^{m}$ is trivial if $p$ is different from 3 , or if $p=3$ and $m \geq 2$ (see Theorem 7.1). Together with Theorem 8.3 this implies:

Corollary 8.4. Let $k$ be a field of positive characteristic $p$, and suppose that $m \geq 2$ if $p=3$. Then for any finite group $G$ whose $p$-Sylow subgroup is cyclic of order $p^{m}$, the canonical Hochschild cohomology class $\gamma_{G}$ is trivial. Hence every graded module over the Tate cohomology algebra $\hat{H}^{*}(G, k)$ is a direct summand of a realizable module.

We investigate more closely the case which is not taken care of by Corollary 8.4. We recall the following characterization of when the restriction to the Sylow subgroup induces an isomorphism in ordinary or Tate cohomology.

Proposition 8.5. Let $k$ be a field of characteristic $p$, and $G$ a finite group, and $P$ a $p$-Sylow subgroup of $G$. Then the following three conditions are equivalent:

(i) The restriction map from the cohomology of $G$ to the cohomology of $P$ is an isomorphism.

(ii) The restriction map $H^{1}(G) \rightarrow H^{1}(P)$ is an isomorphism in dimension 1. 
(iii) The group $G$ is $p$-nilpotent, i.e., there exists a normal subgroup $U \unlhd G$ such that the composite

$$
P \stackrel{\text { incl. }}{\longrightarrow} G \stackrel{\text { proj. }}{\longrightarrow} G / U
$$

is an isomorphism.

Proof. Condition (ii) is a special case of (i). For the implication (ii) $\Rightarrow$ (iii) see 39] or [19 Thm. 7.2.6]. The restriction map from the cohomology of $G$ to the cohomology of its Sylow subgroup $P$ is always injective. If (iii) holds, then it is also surjective since the inclusion $P \rightarrow G$ has a left inverse.

Now let the field $k$ have characteristic 3 and let $G$ by a finite group whose 3-Sylow subgroup is cyclic of order 3 . The Tate cohomology $\hat{H}^{*}(G, k)$ then comes in two flavors, but in both cases the canonical class $\gamma_{G}$ is non-trivial. If $G$ is 3-nilpotent, i.e., if it satisfies the equivalent conditions of Proposition 8.5, then the restriction is an isomorphism from the Tate cohomology of $G$ to that of its Sylow subgroup which takes the canonical class $\gamma_{G}$ to the class $\gamma_{P}$. Otherwise we have:

Theorem 8.6. Let $k$ be a field of characteristic 3 and $G$ a finite group whose 3Sylow subgroup is cyclic of order 3, but which is not 3-nilpotent. Then the Tate cohomology of $G$ is a tensor product of an exterior algebra on a 3-dimensional class $v$ and a Laurent polynomial algebra on a 4-dimensional class $w$,

$$
\hat{H}^{*}(G, k)=\Lambda(v) \otimes k\left[w^{ \pm 1}\right] .
$$

The canonical class $\gamma_{G}$ is represented by the $(3,-1)$-cocycle $m$ of $\Lambda(v) \otimes k\left[w^{ \pm 1}\right]$ which satisfies

$$
m\left(v w^{i} \otimes v w^{j} \otimes v w^{l}\right)=w^{i+j+l+2}
$$

and which vanishes on all other tensor products of monomials in $v$ and $w$.

Proof. Since the 3-Sylow subgroup of $G$ has the trivial intersection property, the Tate cohomology algebra of $G$ restricts isomorphically onto the sub-algebra of $W$ invariant elements in the Tate cohomology of the Sylow subgroup $P$ (see for example [6, Cor. 3.6.19]), where $W=N_{G} P / P$ is the Weyl group of $P$.

The Tate cohomology algebra of $P$ is a tensor product of an exterior algebra on a 1-dimensional class $x$ and a Laurent polynomial algebra on a 2-dimensional class $y$. By Proposition $8.5 H^{1}\left(G, \mathbb{F}_{3}\right)$ is strictly smaller than $H^{1}\left(P, \mathbb{F}_{3}\right) \cong \mathbb{F}_{3}$, so $H^{1}\left(G, \mathbb{F}_{3}\right)$ is trivial. Every element of $W$ acts by +1 or -1 on $H^{1}\left(P, \mathbb{F}_{3}\right)$, and at least one element $w \in W$ must act by -1 on the class $x \in \hat{H}^{1}\left(P, \mathbb{F}_{3}\right)$. The action of $W$ arises from automorphisms of the endomorphism dga which underlies Tate cohomology, so it preserves Massey products. Since $y=\langle x, x, x\rangle$, the element $w$ also acts by -1 on $y$. Hence a monomial $y^{i}$ is $W$-invariant if and only if $i$ is even, and a monomial $x y^{i}$ is $W$-invariant if and only if $i$ is odd. In other words, the subalgebra of $W$-invariant elements is multiplicatively generated by $x y$ and $y^{2}$. So if $v \in \hat{H}^{3}(G, k)$ is the element which restricts to $x y$ and $w \in \hat{H}^{4}(G, k)$ is the element which restricts to $y^{2}$, then the Tate cohomology of $G$ is a tensor product of an exterior algebra on $v$ and a Laurent polynomial algebra on $w$.

By Theorem 8.3, a representing Hochschild $(3,-1)$-cocycle $m_{G}$ for the class $\gamma_{G}$ can be obtained from a representing cocycle $m_{P}$ for the class of the Sylow subgroup by composing with transfer and restriction, i.e.,

$$
m_{G}=[G: P]^{-1} \cdot \operatorname{tr} \circ m_{P} \circ \operatorname{res}^{\otimes 3} .
$$


If we use the cocycle for $\gamma_{P}$ which is given in Theorem 17.1, then we get

$$
\begin{aligned}
{[G: P] \cdot m_{G}\left(v w^{i} \otimes v w^{j} \otimes v w^{l}\right) } & =\operatorname{tr}\left(m_{P}\left(\operatorname{res}\left(v w^{i}\right) \otimes \operatorname{res}\left(v w^{j}\right) \otimes \operatorname{res}\left(v w^{l}\right)\right)\right) \\
& =\operatorname{tr}\left(m_{P}\left(x y^{2 i+1} \otimes x y^{2 j+1} \otimes x y^{2 l+1}\right)\right) \\
& =\operatorname{tr}\left(y^{2(i+j+l)+4}\right)=[G: P] \cdot w^{i+j+l+2},
\end{aligned}
$$

and $[G: P] \cdot m_{G}$ vanishes on all other tensor products of monomials in $v$ and $w$. Dividing by the index $[G: P]$ gives the result.

Example 8.7. Let $k$ be a field of characteristic 3, $G$ a finite group with a 3-Sylow subgroup of order 3 which is not 3 -nilpotent. The smallest example of such a group is the symmetric group on three letters. By Theorem 8.6 the Tate cohomology has the form

$$
\hat{H}^{*}(G, k)=\Lambda(v) \otimes k\left[w^{ \pm 1}\right]
$$

with $|v|=3$ and $|w|=4$, and we have the Massey product relation $\langle v, v, v\rangle=$ $w^{2}$ which holds without indeterminacy. By the same Massey product argument as in Example 7.6. every class in the Tate cohomology of a $k G$-module which is annihilated by $v$ is also divisible by $v$. So all realizable $\hat{H}^{*}(G, k)$-modules, and all summands of those, are actually free. In particular the cyclic $\hat{H}^{*}(G, k)$-module $\hat{H}^{*}(G, k) /(v)$ is not a direct summand of a realizable module.

\section{An APPROXimation FUnCTOR FOR DERIVEd CATEGORIES}

In this section we prove Theorem 6.2 which relates the canonical Hochschild cohomology class of a differential graded algebra $A$ to the realizability obstruction in the derived category of $A$. We also give a functor

$$
\Psi: \operatorname{Mod}-\left(H^{*} A\right) \longrightarrow \mathcal{D}(A)
$$

from the category of graded $H^{*} A$-modules to the derived category of differential graded $A$-modules, together with a natural monomorphism of graded $H^{*} A$-modules $\eta_{X}: X \longrightarrow H^{*}(\Psi X)$. The module $H^{*}(\Psi X)$ is, in a sense, the best possible approximation of $X$ by a realizable module, and it is part of a functorial $\mathcal{D}(A)$-presentation of $X$. The construction is as follows. For brevity, we write $H$ for $H^{*} A$.

A graded $k$-vector space $V$ freely generates a differential graded $A$-module $V \otimes A$. Note that $H^{*}(V \otimes A) \cong V \otimes H$ as right $H$-modules. Let $f_{1}: H^{*} A \rightarrow A$ be a cycle selection homomorphism as in Construction [5.1, the construction of the class $\gamma_{A}$. Let $X$ be a graded $H$-module. We define a map of differential graded $A$-modules

$$
D: X \otimes H^{\otimes n} \otimes A \longrightarrow X \otimes H^{\otimes(n-1)} \otimes A
$$

by the formula

$$
\begin{aligned}
& D\left(x, \zeta_{1}, \ldots, \zeta_{n}, a\right)=\left(x \zeta_{1}, \zeta_{2}, \ldots, \zeta_{n}, a\right) \\
& \quad+\sum_{i=1}^{n-1}(-1)^{i}\left(x, \zeta_{1}, \ldots, \zeta_{i} \zeta_{i+1}, \ldots, \zeta_{n}, a\right)+(-1)^{n}\left(x, \zeta_{1}, \ldots, \zeta_{n-1}, f_{1}\left(\zeta_{n}\right) a\right)
\end{aligned}
$$

Then $D$ induces the bar complex differential $d$ on cohomology. So for example we have

$$
D: X \otimes H \otimes A \longrightarrow X \otimes A, \quad D(x, \zeta, a)=x \zeta \otimes a-x \otimes f_{1}(\zeta) a
$$


and $H^{*}(D)=d: X \otimes H \otimes H \longrightarrow X \otimes H$ sends $x \otimes \zeta_{1} \otimes \zeta_{2}$ to $x \zeta_{1} \otimes \zeta_{2}-x \otimes \zeta_{1} \zeta_{2}$. We define $\Psi X$ as the mapping cone of $D: X \otimes H \otimes A \longrightarrow X \otimes A$. In other words, we set

$$
\Psi X=(X \otimes A) \oplus(X \otimes H \otimes A)[1]
$$

as graded $A$-modules, with differential given by

$$
d_{\Psi X}=\left(\begin{array}{cc}
d & -D \\
0 & -d
\end{array}\right)
$$

(recall that the differential on $(X \otimes H \otimes A)[1]$ is $-d[1])$. Therefore,

$$
d_{\Psi X}\left(\begin{array}{c}
x^{\prime} \otimes a^{\prime} \\
x \otimes \zeta \otimes a
\end{array}\right)=\left(\begin{array}{c}
(-1)^{\left|x^{\prime}\right|} x^{\prime} \otimes d a^{\prime}-x \zeta \otimes a+x \otimes f_{1}(\zeta) a \\
-(-1)^{|x \zeta|} x \otimes \zeta \otimes d a
\end{array}\right) .
$$

There is a distinguished triangle in the derived category $\mathcal{D}(A)$

$$
(\Psi X)[-1] \stackrel{\delta}{\rightarrow} X \otimes H \otimes A \stackrel{D}{\rightarrow} X \otimes A \stackrel{i}{\rightarrow} \Psi X,
$$

where $i$ is the inclusion and $\delta$ is the projection. The triangle (9.2) and the epimorphism

$$
\varepsilon: H^{*}(X \otimes A) \cong X \otimes H \rightarrow X, \quad x \otimes \zeta \longmapsto x \zeta
$$

form a special $\mathcal{D}(A)$-presentation of $X$, in the sense of Definition 3.1

Taking cohomology yields the associated extension of graded $H$-modules

$$
\begin{aligned}
0 \longrightarrow X[-1] \stackrel{\eta_{X}[-1]}{\longrightarrow} & H^{*}(\Psi X)[-1] \\
& \stackrel{H^{*}(\delta)}{\longrightarrow} X \otimes H \otimes H \stackrel{d}{\longrightarrow} X \otimes H \stackrel{\varepsilon}{\rightarrow} X \longrightarrow 0
\end{aligned}
$$

which represents the class $\kappa(X)$. The monomorphism $\eta_{X}: X \longrightarrow H^{*}(\Psi X)$ is given by

$$
\eta_{X}(x)=\left[\begin{array}{c}
x \otimes 1 \\
0
\end{array}\right]
$$

Now we are ready to prove Theorem6.2, For a homomorphism $f: X \rightarrow Y$ of graded $H$-modules we have

$$
\kappa(f)=f \circ \kappa\left(\operatorname{id}_{X}\right) \text { and } f \cup \gamma_{A}=f \circ\left(\operatorname{id}_{X} \cup \gamma_{A}\right) .
$$

Therefore, it suffices to check the case $f=\mathrm{id}_{X}$, the identity map of a graded $H$ module $X$. The canonical class $\gamma_{A}$ of the differential graded algebra $A$ is represented by the Hochschild cocycle $m: H^{\otimes 3} \rightarrow H[-1]$ defined in Construction 5.1] An $H$ -

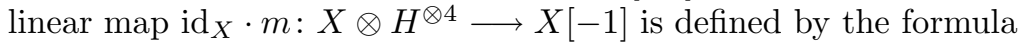

$$
\left(\operatorname{id}_{X} \cdot m\right)\left(x, \zeta_{1}, \zeta_{2}, \zeta_{3}, \zeta_{4}\right)=(-1)^{|x|} x \cdot m\left(\zeta_{1}, \zeta_{2}, \zeta_{3}\right) \cdot \zeta_{4} .
$$

Recall from (4.4) how the cup product pairing between Hochschild cohomology and Ext groups was defined; the following result identifies $\operatorname{id}_{X} \cup \gamma_{A}$ with the Yoneda class of the extension (9.3) which represents $\kappa(X)$. Hence it finishes the proof of Theorem 6.2.

Theorem 9.4. Let $A$ be a differential graded algebra and let $m$ be the Hochschild cocycle of $H=H^{*} A$ as described in Construction 5.1. There exists a commutative 
diagram of graded $H$-modules

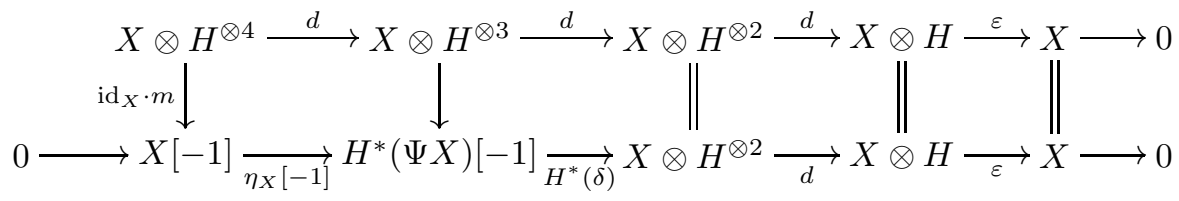

whose rows are exact. Here all maps labeled 'd' are the differentials in the bar complex of $X$. Hence the Yoneda class of the bottom row represents the class $\operatorname{id}_{X} \cup$ $[m]$ in $\operatorname{Ext}_{H}^{3,-1}(X, X)$

Proof. We choose $f_{1}$ and $f_{2}$ as in Construction [5.1, where the Hochschild cocycle $m$ is defined. In (9.1) we have defined homomorphisms of $A$-modules

$$
D: X \otimes H^{\otimes n} \otimes A \longrightarrow X \otimes H^{\otimes(n-1)} \otimes A
$$

which realize the bar complex differential in cohomology. Hence each composite $D D: X \otimes H^{\otimes n} \otimes A \longrightarrow X \otimes H^{\otimes n-2} \otimes A$ induces the trivial map in cohomology, and so it is null-homotopic; an explicit null-homotopy is given by the degree 1 map of graded $A$-modules

$$
\begin{aligned}
\phi: X \otimes H^{\otimes n} \otimes A & \longrightarrow X \otimes H^{\otimes n-2} \otimes A \\
\phi\left(x, \zeta_{1}, \zeta_{2}, \ldots, \zeta_{n}, a\right) & =-(-1)^{\left|x \zeta_{1} \ldots \zeta_{n-2}\right|}\left(x, \zeta_{1}, \ldots, \zeta_{n-2}, f_{2}\left(\zeta_{n-1}, \zeta_{n}\right) x\right) .
\end{aligned}
$$

Indeed, we have

$$
D D=d \circ \phi+\phi \circ d
$$

by direct verification. Using these maps we define a homomorphism of differential graded $A$-modules

$$
\lambda=\left(\begin{array}{c}
\phi \\
D
\end{array}\right): X \otimes H^{\otimes 2} \otimes A \longrightarrow \Psi X[-1]
$$

so that

$$
\lambda\left(x, \zeta_{1}, \zeta_{2}, a\right)=\left(\begin{array}{c}
-(-1)^{|x|} x \otimes f_{2}\left(\zeta_{1}, \zeta_{2}\right) a \\
x \zeta_{1} \otimes \zeta_{2} \otimes a-x \otimes \zeta_{1} \zeta_{2} \otimes a+x \otimes \zeta_{1} \otimes f_{1}\left(\zeta_{2}\right) a
\end{array}\right) .
$$

Using relation (9.5) we have

$$
d_{\Psi X[-1]} \circ \lambda=\left(\begin{array}{cc}
-d & D \\
0 & d
\end{array}\right)\left(\begin{array}{c}
\phi \\
D
\end{array}\right)=\left(\begin{array}{c}
-d \phi+D D \\
d D
\end{array}\right)=\left(\begin{array}{c}
\phi d \\
D d
\end{array}\right)=\lambda \circ d .
$$

Hence $\lambda$ commutes with the differential (recall the the differential on $\Psi X[-1]$ is $\left.-d_{\Psi X}\right)$.

We claim that the diagram of graded $H$-modules

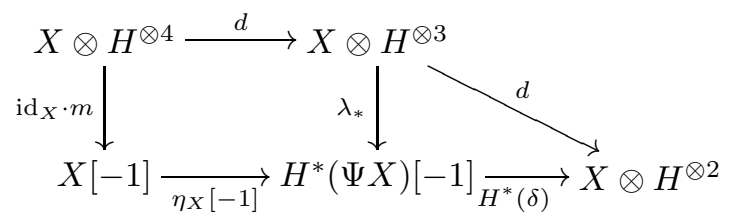

commutes. Since $H^{*}(\delta): H^{*}(\Psi X)[-1] \rightarrow X \otimes H^{\otimes 2}$ is induced by the projection of $\Psi X$ to $(X \otimes H \otimes A)[1]$, the right-hand triangle commutes since the second component of $\lambda$ induces the bar differential on cohomology. 
Commutativity of the left square is seen as follows. Using relations (9.5), we have

$$
\begin{aligned}
\lambda D & =\left(\begin{array}{c}
\phi D \\
D D
\end{array}\right)=\left(\begin{array}{cc}
-d & D \\
0 & d
\end{array}\right)\left(\begin{array}{l}
0 \\
\phi
\end{array}\right)+\left(\begin{array}{c}
-D \phi+\phi D \\
\phi d
\end{array}\right) \\
& =d_{\Psi X[-1]} \circ\left(\begin{array}{l}
0 \\
\phi
\end{array}\right)+\left(\begin{array}{c}
\phi D-D \phi \\
\phi d
\end{array}\right)
\end{aligned}
$$

as maps of graded $A$-modules from $X \otimes H^{\otimes 3} \otimes A$ to $\Psi X[-1]$. Passing to cohomology, the first summand does not contribute, so we have

$$
\lambda_{*} \circ d=(\lambda D)_{*}=\left[\begin{array}{c}
\phi D-D \phi \\
0
\end{array}\right] .
$$

Since $\eta_{X}$ is $H$-linear, we have

$$
\begin{aligned}
\eta_{X} & \left(\left(\operatorname{id}_{X} \cdot m\right)\left(x, \zeta_{1}, \zeta_{2}, \zeta_{3}, 1\right)\right)=(-1)^{|x|} \eta_{X}(x) \cdot m\left(\zeta_{1}, \zeta_{2}, \zeta_{3}\right) \\
= & (-1)^{|x|}\left[x \otimes\left(\begin{array}{c}
f_{2}\left(\zeta_{1}, \zeta_{2}\right) f_{1}\left(\zeta_{3}\right)-f_{2}\left(\zeta_{1}, \zeta_{2} \zeta_{3}\right) \\
+f_{2}\left(\zeta_{1} \zeta_{2}, \zeta_{3}\right)-(-1)^{\left|\zeta_{1}\right|} f_{1}\left(\zeta_{1}\right) f_{2}\left(\zeta_{2}, \zeta_{3}\right)
\end{array}\right)\right] \\
0 & =\left[\begin{array}{c}
\phi D-D \phi \\
0
\end{array}\right]\left(x, \zeta_{1}, \zeta_{2}, \zeta_{3}, 1\right) .
\end{aligned}
$$

Here the last equality comes from substituting the definitions of $D$ and $\phi$. Since both ways around the left square in (9.6) are $H$-linear maps, this completes the proof.

Remark 9.7. For the derived category of a differential graded algebra $A$, we were able to construct a functorial special $\mathcal{D}(A)$-presentation

$$
\begin{aligned}
&\left((\Psi X)[-1] \stackrel{\delta}{\rightarrow} X \otimes\left(H^{*} A\right) \otimes X \stackrel{D}{\rightarrow} X \otimes A \stackrel{i}{\rightarrow} \Psi X,\right. \\
&\left.\varepsilon: H^{*}(X \otimes A) \rightarrow X\right),
\end{aligned}
$$

in the sense of Definition 3.1, of an $H^{*} A$-module $X$. Furthermore, this $\mathcal{D}(A)$ presentation is additive in the module $X$, and it consists of homological functors in the following sense: there is a natural isomorphism $\Psi(X[1]) \cong(\Psi X)[1]$ and for a short exact sequence of $H^{*} A$-modules

$$
0 \longrightarrow X \longrightarrow Y \longrightarrow Z \longrightarrow 0
$$

there is a natural morphism $\Psi Z \rightarrow(\Psi X)[1]$ in $\mathcal{D}(A)$ such that the sequence

$$
\Psi X \longrightarrow \Psi Y \longrightarrow \Psi Z \longrightarrow(\Psi X)[1]
$$

is a distinguished triangle. Indeed, the way we set things up, $\Psi$ takes the short exact sequence (9.9) to a short exact sequence of differential graded $A$-modules. The other two terms in the presentation (9.8) are homological functors by the same argument. In the situation of Section 3 in a general triangulated category $\mathcal{T}$ one cannot hope for $\mathcal{T}$-presentations with such special properties. The construction of (9.8) relies heavily on the underlying abelian category of differential graded $A$-modules which models the derived category $\mathcal{D}(A)$. 


\section{Appendix A. An obstruCtion theory FOR REALIZABILITy}

We are in the same context as in Section 3, $\mathcal{T}$ is a triangulated category with arbitrary coproducts, $N$ is a compact object in $\mathcal{T}$, and we denote by $E=\mathcal{T}(N, N)^{*}$ its graded endomorphism algebra. Given a graded $E$-module $X$, we describe an infinite sequence of classes

$$
\kappa_{n} \in \operatorname{Ext}_{E}^{n, 2-n}(X, X)
$$

for $n \geq 3$, such that each successive class $\kappa_{n}$ is defined if the previous one, $\kappa_{n-1}$, vanishes, and $\kappa_{n}$ depends on a choice. The class $\kappa_{3}$ coincides with $\kappa(X)$ as introduced in Proposition 3.4. If successive choices can be made so that all classes $\kappa_{n}$ are trivial, then $X$ is realizable, i.e., isomorphic to an $E$-module of the form $\mathcal{T}(N, M)^{*}$.

The material in this appendix is, to some extent, folklore in stable homotopy theory, or at least "well-known to the experts". Since we do not know of a published source where this obstruction theory is worked out in the generality of triangulated categories, we give a self-contained account here. However, at least two cases appear in the literature: in [31, Ch. $16 \S 3]$, these obstructions are discussed for the problem of realizing a module over the mod- $p$ Steenrod algebra as the cohomology of a spectrum. If $A$ is a differential graded $k$-algebra, then the theory of $A_{\infty}$-structures shows that a graded module $X$ over the cohomology algebra $H^{*} A$ is realizable by a differential graded $A$-module if and only if the action of $H^{*} A$ on $X$ can be extended to the structure of an $A_{\infty}$-module over the $A_{\infty}$-algebra $H^{*} A$ (see for example [29 Sec. 4]). The obstruction for extending a given $A_{n-1}$-structure on $X$ to an $A_{n}$-structure is (equivalent to) a class in $\operatorname{Ext}_{E}^{n, 2-n}(X, X)$.

Construction A.1. For the constructions in this section we need the mapping telescope (homotopy colimit) construction; compare [12, 2.1] or [34 1.6.4]. Suppose we are given a sequence of morphisms in $\mathcal{T}$,

$$
B_{0} \stackrel{\alpha_{1}}{\longrightarrow} B_{1} \stackrel{\alpha_{2}}{\longrightarrow} \cdots B_{n} \stackrel{\alpha_{n+1}}{\longrightarrow} \cdots .
$$

Then the mapping telescope $\operatorname{Tel}\left(B_{n}\right)$ of the sequence is defined by a choice of distinguished triangle

$$
\operatorname{Tel}\left(B_{n}\right)[-1] \rightarrow \bigoplus_{n=0}^{\infty} B_{n} \stackrel{\text { id-shift }}{\longrightarrow} \bigoplus_{n=0}^{\infty} B_{n} \rightarrow \operatorname{Tel}\left(B_{n}\right)
$$

The map denoted 'shift' takes the summand $B_{n}$ to $B_{n+1}$ via $\alpha_{n+1}$. Since the object $N$ is compact, applying $\mathcal{T}(N,-)^{*}$ to the triangle gives a short exact sequence of E-modules

$$
0 \rightarrow \bigoplus_{n=0}^{\infty} \mathcal{T}\left(N, B_{n}\right)^{*} \stackrel{\text { id-shift }}{\longrightarrow} \bigoplus_{n=0}^{\infty} \mathcal{T}\left(N, B_{n}\right)^{*} \rightarrow \mathcal{T}\left(N, \operatorname{Tel}\left(B_{n}\right)\right)^{*} \rightarrow 0
$$

which shows that $\mathcal{T}\left(N, \operatorname{Tel}\left(B_{n}\right)\right)^{*}$ is the colimit of the sequence of $E$-modules which is realized by (A.2). One should note that the mapping telescope is only welldefined up to non-canonical isomorphism, and that its construction is not functorial, because of the lack of naturality of mapping cones in a triangulated category.

A corollary of the obstruction theory we are about to present is that every graded $E$-module which has projective dimension or injective dimension at most two is actually realizable. Since this corollary has a relatively straightforward proof, 
we present this argument first, even though it is redundant from a strictly logical point of view.

Proposition A.4. Let $X$ be a graded E-module which has projective dimension at most two or injective dimension at most two. Then $X$ is realizable.

Proof. We realize projective modules first. Every projective $E$-module $P$ is isomorphic to the image of an idempotent endomorphism of a graded free module. We choose an $N$-free object $R$ and a morphism $e: R \rightarrow R$ in $\mathcal{T}$ such that $e_{*}: \mathcal{T}(N, R)^{*} \rightarrow \mathcal{T}(N, R)^{*}$ realizes the given idempotent. We define $R_{e}$ as the mapping telescope of the sequence

$$
R \stackrel{e}{\rightarrow} R \stackrel{e}{\rightarrow} \cdots R \stackrel{e}{\rightarrow} \cdots .
$$

Then $R$ decomposes as $R \cong R_{e} \oplus R_{(1-e)}$ (see [12, 3.3] or [34, 1.6.8]), and $\mathcal{T}\left(N, R_{e}\right)^{*}$ is isomorphic to the image of $e_{*}$ in $\mathcal{T}(N, R)^{*}$, i.e., $R_{e}$ realizes the projective $E$ module $P$. Since $R_{e}$ is a direct summand of an $N$-free object (a sum of shifted copies of $N$ ), the map

$$
\mathcal{T}(N,-)^{*}: \mathcal{T}\left(R_{e}, M\right) \longrightarrow \operatorname{Hom}_{E}\left(P, \mathcal{T}(N, M)^{*}\right)
$$

is an isomorphism for every object $M \in \mathcal{T}$. In other words: $E$-homomorphisms from $P$ are uniquely realizable.

Now suppose that $X$ is a graded $E$-module which has projective dimension at most two. Then we have an exact sequence

$$
0 \rightarrow P_{2} \stackrel{d_{2}}{\longrightarrow} P_{1} \stackrel{d_{1}}{\longrightarrow} P_{0} \rightarrow X \rightarrow 0
$$

of graded $E$-modules where $P_{2}, P_{1}$ and $P_{0}$ are projective (possibly trivial). By the previous paragraph we can find a sequence

$$
R_{2} \stackrel{\alpha_{2}}{\longrightarrow} R_{1} \stackrel{\alpha_{1}}{\longrightarrow} R_{0}
$$

of maps in $\mathcal{T}$ such that each $R_{i}$ is a summand of an $N$-free object and $\mathcal{T}\left(N, \alpha_{i}\right)^{*}$ is isomorphic to the map $P_{i} \rightarrow P_{i-1}$ for $i=1,2$. Note that $\alpha_{1} \alpha_{2}=0$ since this composite induces the trivial map after applying $\mathcal{T}(N,-)^{*}$ and since $R_{2}$ is a summand of an $N$-free object.

We complete $\alpha_{2}$ to a distinguished triangle

$$
M_{1}[-1] \rightarrow R_{2} \stackrel{\alpha_{2}}{\longrightarrow} R_{1} \stackrel{\beta_{1}}{\longrightarrow} M_{1} .
$$

Since $\left(\alpha_{2}\right)_{*}=d_{2}$ is injective we obtain a short exact sequence of graded $E$-modules

$$
0 \longrightarrow P_{2} \stackrel{d_{2}}{\longrightarrow} P_{1} \longrightarrow \mathcal{T}\left(N, M_{1}\right)^{*} \longrightarrow 0 .
$$

Since $\alpha_{1} \alpha_{2}=0$ we can choose a map $\gamma: M_{1} \rightarrow R_{0}$ satisfying $\alpha_{1}=\gamma \beta_{1}$. We complete $\gamma$ to a distinguished triangle

$$
M_{0}[-1] \rightarrow M_{1} \stackrel{\gamma}{\rightarrow} R_{0} \stackrel{\beta_{0}}{\longrightarrow} M_{0} .
$$

Since the kernel of $\left(\alpha_{1}\right)_{*}=d_{1}$ is equal to the image of $\left(\alpha_{2}\right)_{*}=d_{2}$, the map

$$
\gamma_{*}: \mathcal{T}\left(N, M_{1}\right)^{*} \rightarrow \mathcal{T}\left(N, R_{0}\right)^{*}
$$

is injective and we obtain another short exact sequence of graded $E$-modules

$$
0 \longrightarrow \mathcal{T}\left(N, M_{1}\right)^{*} \longrightarrow P_{0} \longrightarrow \mathcal{T}\left(N, M_{0}\right)^{*} \longrightarrow 0 \text {. }
$$

Splicing these sequences together, we obtain the projective presentation (A.5) of $X$, and therefore $\mathcal{T}\left(N, M_{0}\right)^{*} \cong X$ as graded $E$-modules. 
The fact that every injective $E$-module is realizable follows from Brown's Representability Theorem in triangulated categories [33]. More precisely, we consider the localizing subcategory $\langle N\rangle$ of $\mathcal{T}$ generated by $N$; this triangulated category is compactly generated since $N$ is compact. If $I$ is an injective $E$-module, then the contravariant functor $\operatorname{Hom}_{E}\left(\mathcal{T}(N,-)^{*}, I\right)$ from $\langle N\rangle$ to the category of abelian groups takes distinguished triangles to long exact sequences and it takes sums to products. So by [33. Thm. 3.1] or [34, 8.4.2] there is a representing object $T_{I}$ of $\langle N\rangle$ and a natural isomorphism

$$
\operatorname{Hom}_{E}\left(\mathcal{T}(N, M)^{*}, I\right) \cong \mathcal{T}\left(M, T_{I}\right)
$$

for $M \in\langle N\rangle$. In particular, the graded $E$-module $\mathcal{T}\left(N, T_{I}\right)^{*}$ is isomorphic to $I$. Similarly, $E$-homomorphisms between injective modules are uniquely realizable by morphisms in $\langle N\rangle$. Given this, the above argument for small projective dimension dualizes to show that modules of injective dimension at most two can be realized. We omit the details.

After this warming up we start with the general obstruction theory for realizing a graded E-module. The crucial concept is that of a Postnikov system; see the following definition. The strategy for realizing a given graded $E$-module $X$ is to build an $N$-exact Postnikov system for which the cokernel of $\left(d_{1}\right)_{*}: \mathcal{T}\left(N, R_{1}\right)^{*} \rightarrow$ $\mathcal{T}\left(N, R_{0}\right)^{*}$ is isomorphic to $X$. Then Proposition A.19 below guarantees that $X$ is realizable, namely by a suitable mapping telescope. In an attempt to build an $N$-exact Postnikov system for $X$ stage by stage, we will run into obstructions

$$
\kappa_{n} \in \operatorname{Ext}_{E}^{n, 2-n}(X, X) .
$$

If all the obstructions are trivial, then we can build the desired Postnikov system and $X$ is realizable.

Definition A.6. Let $\mathcal{T}$ be a triangulated category. A $k$-Postnikov system, for $k \geq 1$, is a collection of morphisms in $\mathcal{T}$
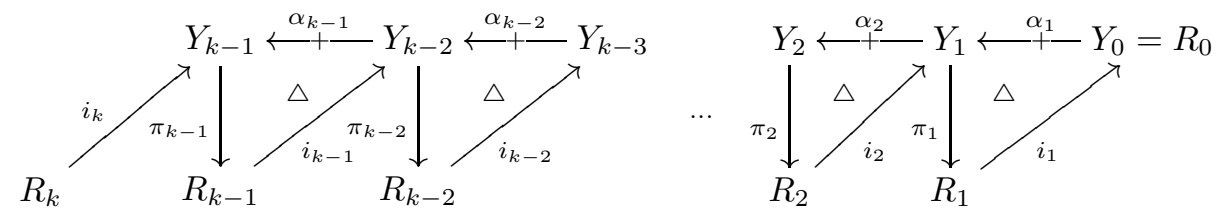

where arrows of the form $\leftarrow+-$ denote morphisms of degree 1 , and where every triangle labeled $\triangle$ is distinguished. For a Postnikov system we define boundary

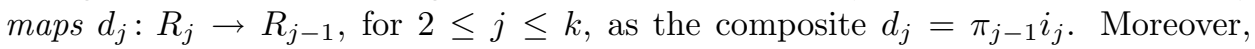
$d_{1}=i_{1}: R_{1} \rightarrow R_{0}$. A $k$-Postnikov system is $N$-exact, for an object $N$ of $\mathcal{T}$, if

(i) the object $R_{i}$ is $N$-free, i.e., a sum of shifted copies of $N$, for $i=0, \ldots, k$,

(ii) and the sequence

$$
\mathcal{T}\left(N, R_{k}\right)^{*} \stackrel{\left(d_{k}\right)_{*}}{\longrightarrow} \mathcal{T}\left(N, R_{k-1}\right)^{*} \stackrel{\left(d_{k-1}\right)_{*}}{\longrightarrow} \cdots \mathcal{T}\left(N, R_{1}\right)^{*} \stackrel{\left(d_{1}\right)_{*}}{\longrightarrow} \mathcal{T}\left(N, R_{0}\right)^{*}
$$

is exact.

A Postnikov system is an " $\infty$-Postnikov system", i.e., a collection of distinguished triangles of the form (A.7) which extends infinitely to the left. A Postnikov system is $N$-exact if the corresponding sequence which extends infinitely to the left is exact. 
Example A.8. The name 'Postnikov system' stems from stable homotopy theory, and we use it because the Postnikov system of a spectrum $X$ is a natural Postnikov system (in the technical sense of Definition A.6) in the stable homotopy category. More generally, suppose the triangulated category $\mathcal{T}$ is endowed with a t-structure [5]. Then the truncations give a Postnikov system, infinite in both directions, of the form

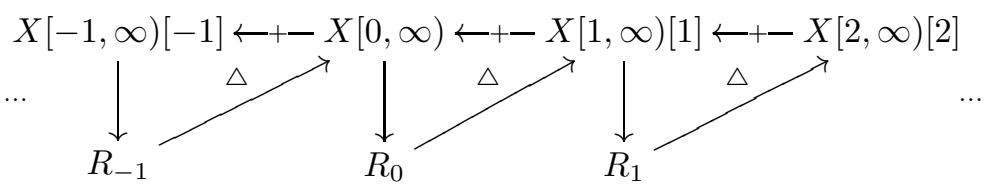

Here $X[i, \infty)$ is the truncation of $X$ below dimension $i$, and where $R_{i}$ is the $i$-fold shift of an object belonging to the heart of the t-structure.

Postnikov systems also arise from filtered objects. For a specific example, consider a differential graded algebra $A$ and a differential graded right $A$-module $M$ together with a filtration by $\mathrm{dg}$ - $A$-submodules

$$
F_{0} \subseteq F_{1} \subseteq \cdots \subseteq F_{n} \subseteq \cdots \subseteq M
$$

Then in the derived category $\mathcal{D}(A)$, we obtain a Postnikov system of the form

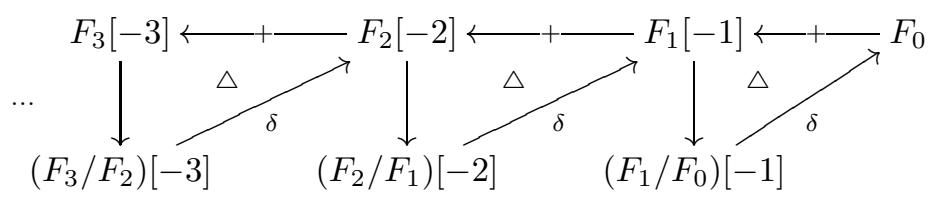

Remark A.11. In every Postnikov system we have $d_{j} d_{j+1}=0$ for $j \geq 1$ since this composition involves two consecutive maps in a distinguished triangle. Thus we have a chain complex

$$
\cdots R_{n} \stackrel{d_{n}}{\longrightarrow} \cdots R_{2} \stackrel{d_{2}}{\longrightarrow} R_{1} \stackrel{d_{1}}{\longrightarrow} R_{0}
$$

in the triangulated category $\mathcal{T}$. However, not every complex can be extended to a Postnikov system. Topologists refer to the obstructions to building a Postnikov system as Toda brackets. For more details on this point of view see for example [42] VI.1] (where a Postnikov system is called a T-system) or [31, Ch. $16 \S 4$ ]. Postnikov systems in a general triangulated category are used for example in [27 §1].

The next lemma collects properties of the system of graded $E$-modules arising from an $N$-exact Postnikov system; the lemma is proved by various diagram chases.

Lemma A.12. Consider an $N$-exact $k$-Postnikov system as in (A.7), with $k \geq 2$.

(i) The given Postnikov system can be extended to an $N$-exact $(k+1)$-Postnikov system if and only if the kernel of $\left(i_{k}\right)_{*}: \mathcal{T}\left(N, R_{k}\right)^{*} \rightarrow \mathcal{T}\left(N, Y_{k-1}\right)^{*}$ is equal to the kernel of $\left(d_{k}\right)_{*}: \mathcal{T}\left(N, R_{k}\right)^{*} \rightarrow \mathcal{T}\left(N, R_{k-1}\right)^{*}$.

(ii) The morphism of graded $E$-modules

$$
\frac{\mathcal{T}\left(N, Y_{i-1}\right)^{*}}{\operatorname{Im}\left(\mathcal{T}\left(N, R_{i}\right)^{*}\right)} \longrightarrow \frac{\mathcal{T}\left(N, Y_{i}\right)^{*}}{\operatorname{Im}\left(\mathcal{T}\left(N, R_{i+1}\right)^{*}\right)}[1]
$$

induced by the morphism $\alpha_{i}: Y_{i-1} \rightarrow Y_{i}[1]$ is an isomorphism for $1 \leq i<$ $k-1$ and an epimorphism for $i=k-1$. 
(iii) The 5-term sequence of graded E-modules

$$
\begin{aligned}
\mathcal{T}\left(N, R_{1}\right)^{*}[1-k] \stackrel{\left(d_{1}\right)_{*}}{\longrightarrow} \mathcal{T}\left(N, R_{0}\right)^{*}[1-k] \stackrel{\alpha_{*}}{\longrightarrow} \mathcal{T}\left(N, Y_{k-1}\right)^{*} \\
\stackrel{\left(\pi_{k-1}\right)_{*}}{\longrightarrow} \mathcal{T}\left(N, R_{k-1}\right)^{*} \stackrel{\left(d_{k-1}\right)_{*}}{\longrightarrow} \mathcal{T}\left(N, R_{k-2}\right)^{*}
\end{aligned}
$$

is exact. Here $\alpha: R_{0}[1-k]=Y_{0}[1-k] \rightarrow Y_{k-1}$ is the composition $\alpha_{k-1} \cdots \alpha_{1}$.

Proof. (i) Since $d_{k}=\pi_{k-1} i_{k}$, the kernel of $\left(i_{k}\right)_{*}: \mathcal{T}\left(N, R_{k}\right)^{*} \rightarrow \mathcal{T}\left(N, Y_{k-1}\right)^{*}$ is always contained in the kernel of $\left(d_{k}\right)_{*}: \mathcal{T}\left(N, R_{k}\right)^{*} \rightarrow \mathcal{T}\left(N, R_{k-1}\right)^{*}$. If the Postnikov system extends to an $N$-exact $(k+1)$-Postnikov system, then the composite $i_{k} d_{k+1}: R_{k+1} \rightarrow Y_{k-1}$ is trivial, and so we have

$$
\operatorname{ker}\left(\left(d_{k}\right)_{*}\right)=\operatorname{Im}\left(\left(d_{k+1}\right)_{*}\right) \subseteq \operatorname{ker}\left(\left(i_{k}\right)_{*}\right) .
$$

Hence the two kernels are equal.

For the converse suppose that the kernels of $\left(i_{k}\right)_{*}$ and $\left(d_{k}\right)_{*}$ coincide. We choose an $N$-free object $R_{k+1}$ and a morphism $d_{k+1}: R_{k+1} \rightarrow R_{k}$ such that the image of $\left(d_{k+1}\right)_{*}$ in $\mathcal{T}\left(N, R_{k}\right)^{*}$ coincides with the kernel of $\left(d_{k}\right)_{*}: \mathcal{T}\left(N, R_{k}\right)^{*} \rightarrow$ $\mathcal{T}\left(N, R_{k-1}\right)^{*}$. By construction, the composite map $d_{k} d_{k+1}: R_{k+1} \rightarrow R_{k-1}$ induces the trivial map after applying $\mathcal{T}(N,-)^{*}$; since $\operatorname{ker}\left(\left(d_{k}\right)_{*}\right)=\operatorname{ker}\left(\left(i_{k}\right)_{*}\right)$, we know that $\left(i_{k} d_{k+1}\right)_{*}: \mathcal{T}\left(N, R_{k+1}\right)^{*} \rightarrow \mathcal{T}\left(N, Y_{k-1}\right)^{*}$ is also trivial. So $i_{k} d_{k+1}=0$ since $R_{k+1}$ is $N$-free. So if we choose a distinguished triangle

$$
Y_{k} \stackrel{\pi_{k}}{\longrightarrow} R_{k} \stackrel{i_{k}}{\longrightarrow} Y_{k-1} \stackrel{\alpha_{k}}{\longrightarrow} Y_{k}[1],
$$

then $d_{k+1}$ can be lifted to a morphism $i_{k+1}: R_{k+1} \rightarrow Y_{k}$ satisfying $d_{k+1}=\pi_{k} i_{k+1}$, and we have constructed an $N$-exact $(k+1)$-Postnikov system.

Part (ii) is proved by a diagram chase which we omit.

For the proof of (iii) we start with the sequence

$$
\mathcal{T}\left(N, R_{1}\right)^{*} \stackrel{\left(d_{1}\right)_{*}}{\longrightarrow} \mathcal{T}\left(N, R_{0}\right)^{*} \stackrel{\left(\alpha_{k-2} \cdots \alpha_{1}\right)_{*}}{\longrightarrow} \frac{\mathcal{T}\left(N, Y_{k-2}\right)^{*}}{\operatorname{Im}\left(\mathcal{T}\left(N, R_{k-1}\right)^{*}\right)}[k-2] \rightarrow 0
$$

which is exact by part (ii), applied $k-2$ times. If we apply $\mathcal{T}(N,-)^{*}$ to the distinguished triangle

$$
R_{k-1}[-1] \stackrel{i_{k-1}}{\longrightarrow} Y_{k-2}[-1] \stackrel{\alpha_{k-1}}{\longrightarrow} Y_{k-1} \stackrel{\pi_{k-1}}{\longrightarrow} R_{k-1}
$$

we get a long exact sequence of graded $E$-modules, a part of which is

$$
0 \rightarrow \frac{\mathcal{T}\left(N, Y_{k-2}\right)^{*}}{\operatorname{Im}\left(\mathcal{T}\left(N, R_{k-1}\right)^{*}\right)}[-1] \stackrel{\left(\alpha_{k-1}\right)_{*}}{\longrightarrow} \mathcal{T}\left(N, Y_{k-1}\right)^{*} \stackrel{\left(\pi_{k-1}\right)_{*}}{\longrightarrow} \mathcal{T}\left(N, R_{k-1}\right)^{*}
$$

Splicing the $(1-k)$ th shift of (A.14) with the exact sequence $($ A.15) gives the first four terms of the exact sequence we are after. For the last spot we exploit that the kernel of $\left(d_{k-1}\right)_{*}$ coincides with the image of $\left(d_{k}\right)_{*}: \mathcal{T}\left(N, R_{k}\right)^{*} \rightarrow \mathcal{T}\left(N, R_{k-1}\right)^{*}$ by $N$-exactness. Since $d_{k}$ factors through $Y_{k-1}$ as $d_{k}=\pi_{k-1} i_{k}$, the image of $\left(d_{k}\right)_{*}$ coincides with the image of $\mathcal{T}\left(N, Y_{k-1}\right)^{*}$.

Now we give the definition of the obstruction class $\kappa_{k+1}$ :

Definition A.16. Consider an $N$-exact $k$-Postnikov system as in A.7) and let $X$ denote the cokernel of the homomorphism

$$
\left(d_{1}\right)_{*}: \mathcal{T}\left(N, R_{1}\right)^{*} \rightarrow \mathcal{T}\left(N, R_{0}\right)^{*} .
$$


By part (iii) of Lemma A.12, the sequence of graded $E$-modules

$$
\begin{aligned}
0 \longrightarrow X[1-k] \stackrel{A}{\longrightarrow} \mathcal{T}\left(N, Y_{k-1}\right)^{*} \stackrel{\left(\pi_{k-1}\right)_{*}}{\longrightarrow} \mathcal{T}\left(N, R_{k-1}\right)^{*} \stackrel{\left(d_{k-1}\right)_{*}}{\longrightarrow} \\
\cdots \mathcal{T}\left(N, R_{1}\right)^{*} \stackrel{\left(d_{1}\right)_{*}}{\longrightarrow} \mathcal{T}\left(N, R_{0}\right)^{*} \longrightarrow X \longrightarrow 0
\end{aligned}
$$

is exact. Here the map $A$ is induced by the composition $\alpha_{k-1} \cdots \alpha_{1}: R_{0}=Y_{0} \rightarrow$ $Y_{k-1}[k-1]$. We denote by $\kappa_{k+1} \in \operatorname{Ext}_{E}^{k+1,1-k}(X, X)$ the Yoneda class of this exact sequence.

The classes $\kappa_{n}$ generalize the obstruction class $\kappa(X)$ which was defined in Section 3 To see this, pick a special presentation $\mathcal{T}$-presentation (cf. Definition 3.1)

$$
\left(B[-1] \stackrel{\delta}{\rightarrow} R_{1} \stackrel{\alpha}{\rightarrow} R_{0} \stackrel{\pi}{\rightarrow} B, \varepsilon: \mathcal{T}\left(N, R_{0}\right)^{*} \rightarrow X\right)
$$

of a graded $E$-module $X$. We can choose an $N$-free object $R_{2}$ and a morphism $d_{2}: R_{2} \rightarrow R_{1}$ such that the image of $\left(d_{2}\right)_{*}$ in $\mathcal{T}\left(N, R_{1}\right)^{*}$ coincides with the kernel of $\alpha_{*}: \mathcal{T}\left(N, R_{1}\right)^{*} \rightarrow \mathcal{T}\left(N, R_{0}\right)^{*}$. The composite $\alpha d_{2}: R_{2} \rightarrow R_{0}$ induces the trivial map after applying $\mathcal{T}(N,-)^{*}$, so $\alpha d_{2}=0$ since $R_{2}$ is $N$-free. Therefore, $d_{2}$ can be lifted to a morphism $i_{2}: R_{2} \rightarrow B[-1]$. This data yields an $N$-exact 2-Postnikov system

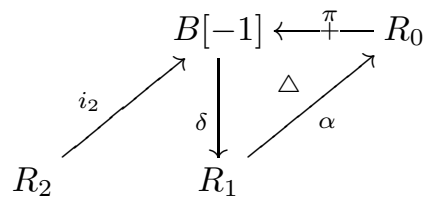

The exact sequence (A.17) which defines the obstruction class $\kappa_{3} \in \operatorname{Ext}_{E}^{3,-1}(X, X)$ is the associated extension of the $\mathcal{T}$-presentation. So $\kappa_{3}$ coincides with $\kappa(X)$ as defined in Proposition 3.4 .

Lemma A.18. If the obstruction class $\kappa_{k+1}$ of an $N$-exact $k$-Postnikov system is trivial, then there exists an $N$-exact $(k+1)$-Postnikov system whose $(k-1)$ Postnikov system agrees with that of the given k-Postnikov system.

Proof. In the exact sequence (A.17) which defines $\kappa_{k+1}$, the $E$-modules $\mathcal{T}\left(N, R_{i}\right)^{*}$ are free for $i=0, \ldots, k-1$. Hence the class $\kappa_{k+1}$ is trivial if and only if the monomorphism $A: X[1-k] \rightarrow \mathcal{T}\left(N, Y_{k-1}\right)^{*}$ admits a retraction $r: \mathcal{T}\left(N, Y_{k-1}\right)^{*} \rightarrow$ $X[1-k]$. Since $R_{k}$ is $N$-free, the $E$-homomorphism $A \circ r \circ\left(i_{k}\right)_{*}: \mathcal{T}\left(N, R_{k}\right)^{*} \rightarrow$ $\mathcal{T}\left(N, Y_{k-1}\right)^{*}$ is realized by a unique morphism $\beta: R_{k} \rightarrow Y_{k-1}$ in $\mathcal{T}$. The composite $\pi_{k-1} \beta: R_{k} \rightarrow R_{k-1}$ is trivial after applying $\mathcal{T}(N,-)^{*}$, so $\pi_{k-1} \beta=0$, again since $R_{k}$ is $N$-free.

If we replace the map $i_{k}: R_{k} \rightarrow Y_{k-1}$ by $\bar{i}_{k}=i_{k}-\beta$, then the modified $k$ Postnikov system

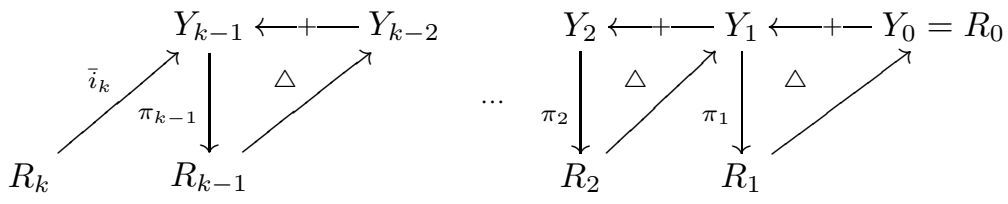

is again $N$-exact since $\pi_{k-1} \bar{i}_{k}=\pi_{k-1} i_{k}=d_{k}$.

What we have gained now is that the kernel of $\left(\bar{i}_{k}\right)_{*}: \mathcal{T}\left(N, R_{k}\right)^{*} \rightarrow \mathcal{T}\left(N, Y_{k-1}\right)^{*}$ is equal to the kernel of $\left(d_{k}\right)_{*}: \mathcal{T}\left(N, R_{k}\right)^{*} \rightarrow \mathcal{T}\left(N, R_{k-1}\right)^{*}$ : suppose that $x \in$ 
$\mathcal{T}\left(N, R_{k}\right)^{*}$ is annihilated by $\left(d_{k}\right)_{*}$. Then $\left(\pi_{k-1}\right)_{*}\left(i_{k}\right)_{*}(x)=\left(d_{k}\right)_{*}(x)=0$, so $\left(i_{k}\right)_{*}(x)=A(y)$ for some $y \in X[1-k]$. But then

$$
\left(\bar{i}_{k}\right)_{*}(x)=\left(i_{k}-\beta\right)_{*}(x)=(\mathrm{id}-A \circ r)\left(\left(i_{k}\right)_{*}(x)\right)=(\mathrm{id}-A \circ r)(A(y))=0 .
$$

So by Lemma A.12(i), the modified $k$-Postnikov system can be extended to an $N$-exact $(k+1)$-Postnikov system.

Proposition A.19. Let $N$ be a compact object of the triangulated category $\mathcal{T}$. For every $N$-exact Postnikov system, the cokernel of the homomorphism

$$
\left(d_{1}\right)_{*}: \mathcal{T}\left(N, R_{1}\right)^{*} \rightarrow \mathcal{T}\left(N, R_{0}\right)^{*}
$$

is a realizable E-module.

Proof. We claim that the cokernel of $\left(d_{1}\right)_{*}$ is isomorphic to the colimit of the sequence of graded $E$-modules

$$
\mathcal{T}\left(N, Y_{0}\right)^{*} \stackrel{\left(\alpha_{1}\right)_{*}}{\longrightarrow} \mathcal{T}\left(N, Y_{1}[1]\right)^{*} \stackrel{\left(\alpha_{2}\right)_{*}}{\longrightarrow} \cdots \mathcal{T}\left(N, Y_{n}[n]\right)^{*} \stackrel{\left(\alpha_{n+1}\right)_{*}}{\longrightarrow} \cdots
$$

This implies the result since the colimit is realizable, namely by the mapping telescope of the sequence of morphisms $\alpha_{k}: Y_{k-1}[k-1] \rightarrow Y_{k}[k]$; compare A.1.

Since $\alpha_{n} i_{n}=0$, the composite map

$$
\mathcal{T}\left(N, R_{n}\right)^{*} \stackrel{\left(i_{n}\right)_{*}}{\longrightarrow} \mathcal{T}\left(N, Y_{n-1}\right)^{*} \stackrel{\left(\alpha_{n}\right)_{*}}{\longrightarrow} \mathcal{T}\left(N, Y_{n}[1]\right)^{*}
$$

is trivial for all $n \geq 1$. So the colimit does not change if we factor out the image of $\mathcal{T}\left(N, R_{n+1}\right)^{*}$ in $\mathcal{T}\left(N, Y_{n}\right)^{*}$. In other words, the colimit of (A.20) maps isomorphically to the colimit of the sequence

$$
\begin{aligned}
\operatorname{coker}\left(\left(d_{1}\right)_{*}\right)=\frac{\mathcal{T}\left(N, Y_{0}\right)^{*}}{\operatorname{Im}\left(\mathcal{T}\left(N, R_{1}\right)^{*}\right)} & \longrightarrow \frac{\mathcal{T}\left(N, Y_{1}\right)^{*}}{\operatorname{Im}\left(\mathcal{T}\left(N, R_{2}\right)^{*}\right)}[1] \\
\longrightarrow \cdots & \longrightarrow \frac{\mathcal{T}\left(N, Y_{n}\right)^{*}}{\operatorname{Im}\left(\mathcal{T}\left(N, R_{n+1}\right)^{*}\right)}[n] \longrightarrow \cdots .
\end{aligned}
$$

By part (ii) of Lemma A.12 the latter sequence consists of isomorphisms, so the cokernel of $\left(d_{1}\right)_{*}$ is indeed isomorphic, as a graded $E$-module, to the colimit of the sequence A.20.

Conclusion. If we are given a graded $E$-module $X$, we can always find an $N$ exact 2-Postnikov system for which the cokernel of $\left(d_{1}\right)_{*}: \mathcal{T}\left(N, R_{1}\right)^{*} \rightarrow \mathcal{T}\left(N, R_{0}\right)^{*}$ is isomorphic to $X$ (see the discussion preceding Lemma A.18). For $k \geq 2$, an

$N$-exact $k$-Postnikov system defines an obstruction class $\kappa_{k+1} \in \operatorname{Ext}_{E}^{k+1,1-\bar{k}}(X, X)$ (Definition A.16). Lemma A.18 says that if $\kappa_{k+1}=0$, then after perturbing the last morphism $i_{k}: R_{k} \rightarrow Y_{k-1}$ if necessary, the $k$-Postnikov system can be extended to an $N$-exact $(k+1)$-Postnikov system. The extension is not unique, but every choice of extension defines a new obstruction class $\kappa_{k+2}$. If we can make inductive choices so that all classes $\kappa_{k}$ are trivial, then we have constructed an $N$-exact Postnikov system. Proposition A.19 then implies that the $E$-module $X$ is realizable.

\section{REFERENCES}

[1] M. Auslander and S. O. Smalø, Preprojective modules over artin algebras, J. Algebra 66 (1980), 61-122. MR 83a:16039

[2] H.-J. Baues, On the cohomology of categories, universal Toda brackets and homotopy pairs, $K$-Theory 11 (1997), no. 3, 259-285. MR 98h:55020 
[3] H.-J. Baues and W. Dreckmann, The cohomology of homotopy categories and the general linear group, $K$-Theory 3 (1989), no. 4, 307-338. MR 91d:18008

[4] H.-J. Baues and A. Tonks, On sum-normalised cohomology of categories, twisted homotopy pairs and universal Toda brackets, Quarterly Journal of Math (Oxford) 47 (1996), no. 188, 405-433. MR 98k:18010

[5] A. A. Beĭlinson, J. Bernstein, and P. Deligne, Faisceaux pervers, Analysis and topology on singular spaces, I (Luminy, 1981), Soc. Math. France, Paris, 1982, pp. 5-171. MR 86g:32015

[6] D. J. Benson, Representations and Cohomology I: Basic representation theory of finite groups and associative algebras, Cambridge Studies in Advanced Mathematics, vol. 30, Cambridge University Press, 1991. MR 92m:20005

[7] _ Complexity and varieties for infinite groups, I, J. Algebra 193 (1997), 260-287. MR 99a:20054

[8] _ Complexity and varieties for infinite groups, II, J. Algebra 193 (1997), 288-317. MR 99a:20054

[9] D. J. Benson and J. F. Carlson, Products in negative cohomology, J. Pure \& Applied Algebra 82 (1992), 107-129. MR 93i:20058

[10] D. J. Benson and G. Ph. Gnacadja, Phantom maps and purity in modular representation theory, II, Algebras and Representation Theory 4 (2001), 395-404. MR 2002j:20017

[11] A. J. Berrick and A. A. Davydov, Splitting of Gysin extensions, Algebr. Geom. Topol. 1 (2001), 743-762. MR 2003a:55021

[12] M. Bökstedt and A. Neeman, Homotopy limits in triangulated categories, Compositio Math. 86 (1993), no. 2, 209-234. MR 94f:18008

[13] J. F. Carlson, Modules and group algebras, Lectures in Mathematics, ETH Zürich, Birkhäuser, 1996. MR 97c:20013

[14] H. Cartan and S. Eilenberg, Homological algebra, Princeton Mathematical Series, no. 19, Princeton Univ. Press, 1956. MR 17:1040e

[15] D. Christensen, B. Keller, and A. Neeman, Failure of Brown representability in derived categories, Topology 40 (2001), 1339-1361.

[16] J. Cornick and P. H. Kropholler, Homological finiteness conditions for modules over strongly group-graded rings, Math. Proc. Camb. Phil. Soc. 120 (1996), 43-54. MR 96m:16009

[17] _ Homological finiteness conditions for modules over group algebras, J. London Math. Soc. 58 (1998), 49-62. MR 99k:20105

[18] P. Deligne, Cohomologie étale, Springer-Verlag, Berlin/New York, Berlin, 1977, Séminaire de Géométrie Algébrique du Bois-Marie SGA $4 \frac{1}{2}$, Avec la collaboration de J. F. Boutot, A. Grothendieck, L. Illusie et J. L. Verdier, Lecture Notes in Mathematics, Vol. 569. MR 57:3132

[19] L. Evens, The cohomology of groups, The Clarendon Press Oxford University Press, New York, 1991, Oxford Science Publications. MR 93i:20059

[20] P. Freyd, Stable homotopy, Proceedings of the conference on categorical algebra (La Jolla, 1965), Springer-Verlag, Berlin/New York, 1966, pp. 121-172. MR 35:2280

[21] S. I. Gelfand and Yu. I. Manin, Methods of homological algebra, Springer-Verlag, Berlin/New York, 1996. MR 97j:18001

[22] M. Gerstenhaber, The cohomology structure of an associative ring, Ann. of Math. (2) 78 (1963), 267-288. MR 28:5102

[23] François Goichot, Homologie de Tate-Vogel équivariante, J. Pure Appl. Algebra 82 (1992), 39-64. MR 94d:55014

[24] D. Happel, On the derived category of a finite-dimensional algebra, Comment. Math. Helvetici 62 (1987), 339-389. MR 89c:16029

[25] T. V. Kadeishvili, The algebraic structure in the homology of an $A(\infty)$-algebra, Soobshch. Akad. Nauk Gruzin. SSR 108 (1982), no. 2, 249-252 (1983). MR 84k:55009

[26] _ The structure of the $A(\infty)$-algebra, and the Hochschild and Harrison cohomologies, Trudy Tbiliss. Mat. Inst. Razmadze Akad. Nauk Gruzin. SSR 91 (1988), 19-27. MR 91a:18016

[27] M. M. Kapranov, On the derived categories of coherent sheaves on some homogeneous spaces, Invent. Math. 92 (1988), no. 3, 479-508. MR 89g:18018

[28] B. Keller, Deriving DG categories, Ann. Sci. École Norm. Sup. (4) 27 (1994), no. 1, 63-102. MR 95e:18010 
[29] Introduction to A-infinity algebras and modules, Homology, Homotopy and Applications 3 (2001), 1-35.

[30] P. H. Kropholler, Hierarchical decompositions, generalized Tate cohomology, and groups of type $F P_{\infty}$, Proc. Edin. Conf. Geometric Group Theory 1993 (A. Duncan, N. Gilbert, and J. Howie, eds.), Cambridge University Press, 1994. MR 96c:20097

[31] H. R. Margolis, Spectra and the Steenrod algebra, North Holland, Amsterdam, 1983. MR 86j:55001

[32] G. Mislin, Tate cohomology for arbitrary groups via satellites, Topology and its Applications 56 (1994), 293-300. MR 95c:20072

[33] A. Neeman, The Grothendieck duality theorem via Bousfield's techniques and Brown representability, J. Amer. Math. Soc. 9 (1996), no. 1, 205-236. MR 96c:18006

[34] _ Triangulated categories, Princeton University Press, Princeton, NJ, 2001. MR 2001k:18010

[35] J. Rickard, Idempotent modules in the stable category, J. London Math. Soc. 178 (1997), 149-170. MR 98d:20058

[36] C. M. Ringel, Tame algebras and integral quadratic forms, Lecture Notes in Mathematics, vol. 1099, Springer-Verlag, Berlin/New York, 1984. MR 87f:16027

[37] N. Spaltenstein, Resolutions of unbounded complexes, Compositio Math. 65 (1988), 121-154. MR 89m:18013

[38] J. D. Stasheff, Homotopy associativity of H-spaces. II, Trans. Amer. Math. Soc. 108 (1963), 293-312. MR 28:1623

[39] J. Tate, Nilpotent quotient groups, Topology 3 (1964), 109-111, suppl. 1. MR 28:4032

[40] J.-L. Verdier, Des catégories dérivées des catégories abéliennes, Astérisque (1996), no. 239, xii+253 pp. (1997), with a preface by Luc Illusie, edited and with a note by Georges Maltsiniotis. MR 98c: 18007

[41] C. A. Weibel, Introduction to homological algebra, Cambridge Studies in Advanced Mathematics, vol. 38, Cambridge University Press, 1994. MR 95f:18001

[42] G. W. Whitehead, Recent advances in homotopy theory, American Mathematical Society, Providence, R.I., 1970, Conference Board of the Mathematical Sciences Regional Conference Series in Mathematics, No. 5. MR 46:8208

Department of Mathematics, University of Georgia, Athens, Georgia 30602

E-mail address: djb@byrd.math.uga.edu

Department of Pure Mathematics, University of Leeds, Leeds LS2 9JT, United KingDOM

E-mail address: henning@maths.leeds.ac.uk

Current address: Institut für Mathematik, Universität Paderborn, D-33095 Paderborn, Germany

E-mail address: hkrause@math.upb.de

SFB 478 Geometrische Strukturen in Der Mathematik, Westfälische Wilhelms-UniVersität Münster, Hittorfstr. 27, 48149 Münster, Germany

E-mail address: sschwede@mathematik.uni-muenster.de 